\title{
Data report: consolidation characteristics of sediments from IODP Expedition 308, Ursa Basin, Gulf of Mexico'
}

\author{
H. Long, ${ }^{2,3}$ P.B. Flemings, ${ }^{4}$ J.T. Germaine, ${ }^{5}$ D.M. Saffer, ${ }^{6}$ and B. Dugan ${ }^{7}$
}

\section{Chapter contents}

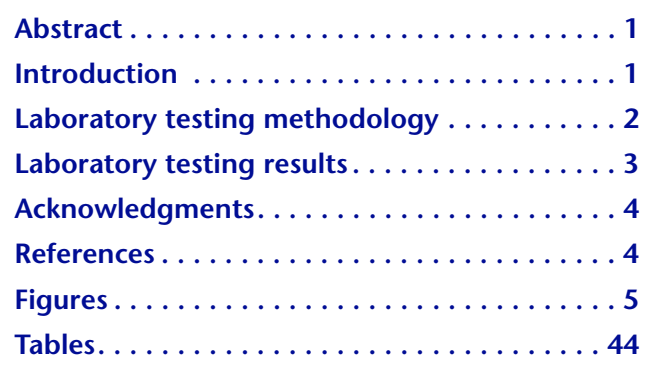

${ }^{1}$ Long, H., Flemings, P.B., Germaine, J.T., Saffer, D.M., and Dugan, B., 2008. Data report: consolidation characteristics of sediments from IODP Expedition 308, Ursa Basin, Gulf of Mexico. In Flemings, P.B., Behrmann, J.H., John, C.M., and the Expedition 308 Scientists, Proc. IODP, 308: College Station, TX (Integrated Ocean Drilling Program Management International, Inc.). doi:10.2204/iodp.proc.308.204.2008

${ }^{2}$ Department of Energy and Mineral Engineering, The Pennsylvania State University, University Park PA 16802, USA.

${ }^{3}$ Present address: ExxonMobil Upstream Research Company, Houston, TX 77098.

${ }^{4}$ John A. and Katherine G. Jackson School of Geosciences, The University of Texas at Austin, Austin TX 78712, USA. pflemings@jsg.utexas.edu ${ }^{5}$ Department of Civil and Environmental Engineering, Massachusetts Institute of Technology, Cambridge MA 02139, USA. ${ }^{6}$ Department of Geosciences, The Pennsylvania State University, University Park, PA 16802, USA. ${ }^{7}$ Department of Earth Science, Rice University, Houston TX 77005, USA.

\section{Abstract}

We conducted constant rate of strain consolidation tests on 9 samples from Integrated Ocean Drilling Program Site U1322 and 23 samples from Site U1324 in three laboratories (Massachusetts Institute of Technology, Pennsylvania State University, and Rice University) to obtain the consolidation properties of the sediment, as well as determine the stress history of the sites. The sediments recovered from above 200 meters below seafloor (mbsf) at both sites have similar consolidation properties. The compression index $\left(C_{\mathrm{c}}\right)$ ranges from 0.1508 to $0.5052 . C_{\mathrm{c}}$ decreases with void ratio at both sites. The expansion index $\left(C_{\mathrm{e}}\right)$ ranges from 0.0153 to 0.1144 and decreases with void ratio at both sites. The in situ hydraulic conductivity $\left(K_{\mathrm{i}}\right)$ ranges from $2.18 \times 10^{-11}$ to $6.38 \times 10^{-10}$ $\mathrm{m} / \mathrm{s} . K_{\mathrm{i}}$ decreases with depth. The $e-\log \left(K_{\mathrm{i}}\right)$ relation has different slopes for sediments above and below $300 \mathrm{mbsf}$ at Site U1324. The coefficient of consolidation $\left(c_{v}\right)$ ranges from $1.5 \times 10^{-8}$ to $4.2 \times$ $10^{-7} \mathrm{~m}^{2} / \mathrm{s}$. $c_{\mathrm{v}}$ increases with depth for the sediments above 200 mbsf at both sites and shows no clear trend for the sediments below 200 mbsf at Site U1324. The preconsolidation pressure $\left(P_{c}^{\prime}\right)$ is significantly less than the hydrostatic vertical effective stress $\left(\sigma_{v h}^{\prime}\right)$ at both sites, which suggests that Ursa sediments are overpressured.

\section{Introduction}

Understanding overpressure, fluid flow, and sediment compression behavior is critical for evaluating the stability of continental slopes. Integrated Ocean Drilling Program (IODP) Expedition 308 was aimed at testing a multidimensional flow model by examining how physical properties, pressure, temperature, and pore fluid composition vary within low-permeability mudstones that overlie a permeable and overpressured aquifer (see the "Expedition 308 summary" chapter). We drilled, logged, cored, and made in situ measurements in a region of very rapid Pleistocene sedimentation: the Ursa Basin (Fig. F1).

We took whole-core geotechnical samples for shore-based consolidation tests (Table T1). Consolidation tests simulate how porosity evolves with effective stress under one-dimensional gravitational compaction caused by sedimentation. The transition from recompression to virgin compression behavior provides an estimate of the maximum in situ effective stress the sample has undergone 
(Becker et al., 1987; Casagrande, 1936). The experiments also provide insight into how permeability evolves with burial and compression.

Consolidation properties were determined from results of constant rate of strain consolidation (CRSC) tests on intact samples.

\section{Laboratory testing methodology}

\section{Sample handling and preparation}

The coring techniques include the advanced piston corer (APC) and extended core barrel (XCB) systems (Table T1). These standard coring systems and their characteristics are summarized in Technical Note 31 of the Ocean Drilling Program (Graber et al., 2002). The sample was not extruded from the core liner on board the drilling ship. Whole-core samples were capped and sealed in wax to maintain natural saturation during refrigerated storage prior to the experiments. For the experiments, each sample was removed from the wax-sealed liner and subsampled with a sharp cutting shoe (at Pennsylvania State University [PSU]) or a trimming jig (at Massachusetts Institute of Technology [MIT] and Rice University [Rice]).

\section{Sample descriptions}

Table T1 illustrates all of the samples tested in this study including sample depth, type of coring used to acquire the sample, what analyses were done, and where the analyses were done. Most of the PSU and MIT samples were X-rayed at MIT's radiography facility in order to select undisturbed portions of the core for experiments and to assess the presence of inclusions and variation in fabric. The Rice samples were $\mathrm{X}$-rayed by Fugro in order to select undisturbed portions for experiments and to identify layering or inhomogeneities. Core X-ray data can be found in $\mathrm{H}$. Nelson et al. (unpubl. data).

All samples were taken from whole core, not core catchers. Sample locations are illustrated in Table T1. Quality of samples generally decreases with depth and many samples had significant deformation caused by the coring process. Table $\mathrm{T} 1$ indicates which samples were recovered by the APC and XCB systems. During APC coring, two different cutting shoes were used: the "Fugro" cutting shoe and the "IODP-APC" cutting shoe. The Fugro cutting shoe has a thinner kerf than the IODP-APC cutting shoe; therefore, less deformation during coring was expected.

Grain size analysis documents that all tested samples are silty clays containing 50\%-70\% clay-sized grains $(<2 \mu \mathrm{m})$, except samples from Section 308-U1324C-
7H-1 (405 meters below seafloor [mbsf]), which are clayey silt with 30\% clay-sized grains (Sawyer et al., this volume). The mineralogic composition of the silty clay samples is similar. Quantitative X-ray mineralogy shows that illite and smectite are the dominant minerals and together comprise $37 \%-60 \%$ of the bulk rock weight. Analysis of the clay-sized fraction $(<2 \mu \mathrm{m})$ by subjective analysis of oriented mounts shows that $80 \%-90 \%$ of the mixed-layer interlayers are composed of smectite.

\section{Index properties}

Two water contents were measured in the consolidation test: $w_{\mathrm{c}}$ and $w_{\mathrm{n}} \cdot w_{\mathrm{c}}$ is the water content measured on the leftover trimmings during sample preparation. $w_{\mathrm{n}}$ is the water content measured on the test specimen itself. We measured the water content by oven-drying the samples. Water content is calculated by taking the difference in the weight of the sample before and after oven-drying and dividing this difference by the oven-dried weight.

In the laboratory, water content was measured on the trimmings from the specimens and on the specimens themselves. The water content of the trimmings were generally lower $(\sim 3 \%)$ than the water content of the samples. We compared the water content measured on the specimens with the shipboard measurements of moisture and density (MAD). This comparison is difficult because the sampling frequency and quality of the MAD data are variable. We find that at 15 locations the difference between the laboratory-derived water content and the MAD measurements are within 5 units in water content. There is no systematic difference.

\section{Constant rate of strain consolidation testing}

CRSC tests were conducted at three laboratories (MIT, PSU, and Rice) in general accordance with American Society for Testing and Materials (ASTM) D4186 guidelines (ASTM International, 2006). As the name implies, during CRSC tests the sample is deformed at a constant strain rate. This has several advantages relative to traditional step-loading tests. It provides continuous loading data, which provide a much more detailed view of compression behavior. In contrast with incremental loading, data points are obtained by doubling stress levels: behavior must be inferred between these points. In addition, continuous measurements of the flow properties are obtained (both the hydraulic conductivity $[K]$ and the coefficient of consolidation $\left.\left[c_{\mathrm{v}}\right]\right)$. Finally, $K$ is calculated directly (Equation 2), as opposed to the incremental consolidation test where it is indirectly 
calculated from the coefficient of consolidation $\left(c_{v}\right)$ and the frame compressibility $\left(m_{\mathrm{v}}\right)$.

The dimensions of the specimen are slightly different between the three laboratories. MIT specimens were $5.95-6.35 \mathrm{~cm}$ in diameter with an initial height of $2.35 \mathrm{~cm}$. PSU specimens were $5 \mathrm{~cm}$ in diameter with an initial height of $2.0 \mathrm{~cm}$. Rice specimens were 5.09 $\mathrm{cm}$ in diameter with an initial height of $2.41 \mathrm{~cm}$.

Specimens were laterally confined with a steel ring. Prior to testing, specimens were saturated with deaired distilled water and back-pressured to 300-425 $\mathrm{kPa}$ for $24 \mathrm{~h}$ to drive any gases present into solution. We applied a constant rate of strain using a computer-controlled load frame, with the sample base undrained and sample top open to the backpressure. We continuously monitored sample height $(H$, in millimeters), applied vertical stress $\left(\sigma_{v}\right.$, in $\left.\mathrm{kPa}\right)$, and basal pore pressure $(u$, in $\mathrm{kPa})$. At PSU, an axial load of $50 \mathrm{kN}$ could be applied by a mechanical load frame and $5 \mathrm{~cm}$ diameter samples were run; therefore, the maximum vertical stress was $25.5 \mathrm{MPa}$. Backpressure was $0.3 \mathrm{MPa}$; therefore, the maximum effective stress that could be achieved was $\sim 25.2$ MPa. The MIT and Rice apparatuses were capable of lower total effective stresses. Most MIT experiments were run to $\sim 2 \mathrm{MPa}$. Most Rice experiments were completed at effective stresses near $4 \mathrm{MPa}$. For this reason, the MIT and Rice apparatuses were used on the shallower samples.

The vertical effective stress $\left(\sigma_{\mathrm{v}}^{\prime}\right)$, hydraulic conductivity $(K)$, compressibility $\left(m_{\mathrm{v}}\right)$, coefficient of consolidation $\left(c_{\mathrm{v}}\right)$, and strain energy density (SED) were calculated using the following equations (ASTM, 2006; Tan et al., 2006):

$$
\begin{gathered}
\sigma_{v}^{\prime}=\sigma_{v}-\frac{2}{3} \cdot \Delta u, \\
K=\frac{\frac{d \varepsilon}{d t} \cdot H_{0} \cdot H \cdot \gamma_{w}}{2 \cdot \Delta u}, \\
m_{v}=\frac{\Delta \varepsilon}{\Delta \sigma_{v}^{\prime}{ }^{\prime}} \\
c_{v}=\frac{K}{m_{v} \cdot \gamma_{w}}, \text { and } \\
\text { SED }=\frac{\sigma_{v_{i}-1}+\sigma_{v_{i}}^{\prime}}{2} \cdot \operatorname{Ln}\left(\frac{1-\varepsilon_{i-1}}{1-\varepsilon_{i}}\right) .
\end{gathered}
$$

Variables are defined in Table T2.

\section{Laboratory testing results}

We conducted CRSC tests on 9 samples from Site U1322 and 23 samples from Site U1324 in two laboratories (MIT and PSU). Table T3 summarizes the details of each CRSC test. Figures F4, F5, F6, F7, F8, F9, F10, F11, F12, F13, F14, F15, F16, F17, F18, F19, F20, F21, F22, F23, F24, F25, F26, F27, F28, F29, F30, F31, F32, F33, F34, and F35 show the consolidation curves in both $e-\log \left(\sigma_{\mathrm{v}}^{\prime}\right)$ and $\varepsilon-\log \left(\sigma_{\mathrm{v}}^{\prime}\right)$, normalized excess pore pressure, coefficient of consolidation $\left(c_{\mathrm{v}}\right)$, SED, and hydraulic conductivity $(K)$ for each CRSC test. The CRSC data sheet, which includes 12 columns (Table T4), can be found in Microsoft Excel format in "Supplementary material."

The compression index $\left(C_{c}\right)$ refers to the slope of the normally consolidated portion of the compression curve in $e$-log $\left(\sigma_{\mathrm{v}}^{\prime}\right)$ space (Fig. F2). The compression behavior of the samples is similar at Sites U1322 and U1324 (Fig. F36). The measured values of $c_{c}$ range from 0.1508 to $0.5052 . c_{c}$ decreases with void ratio at both sites (Fig. F36). The expansion index $\left(c_{\mathrm{e}}\right)$ refers to the slope of the unloading portion of the compression curve in $e$-log $\left(\sigma_{\mathrm{v}}^{\prime}\right)$ space. It ranges from 0.0153 to 0.1144 and also decreases with void ratio (Fig. F36). It must be noted that the expansion index varies with the amount of unloading that occurs. As such, the quoted expansion indexes are for unloading to an overconsolidation ratio of 10 .

The in situ hydraulic conductivity $\left(K_{\mathrm{i}}\right)$ is obtained by extrapolating the linear portion of the $e-\log (K)$ relation to the in situ void ratio. Values of $K_{\mathrm{i}}$ range from $2.18 \times 10^{-11}$ to $6.38 \times 10^{-10} \mathrm{~m} / \mathrm{s}$. $K_{\mathrm{i}}$ decreases with depth (Fig. F37). The $e_{\mathrm{i}}-\log \left(K_{\mathrm{i}}\right)$ relations for sediments above and below 300 mbsf have different slopes (Fig. F37). $K_{\mathrm{i}}$ of the clayey silt sample ( $405.81 \mathrm{mbsf}$ ) is significantly higher than those of the silty clay samples, which reflects the lithology difference and stands out on the $e_{\mathrm{i}}-\log \left(K_{\mathrm{i}}\right)$ plot (Fig. F37).

The coefficient of consolidation, $c_{\mathrm{v}}$, ranges from 1.5 $\times 10^{-8}$ to $4.2 \times 10^{-7} \mathrm{~m}^{2} / \mathrm{s}$ (Fig. F38). $c_{\mathrm{v}}$ increases with depth for the sediments above 200 mbsf at both sites and shows no clear trend for the sediments below 200 mbsf. $c_{v}$ of the clayey silt sample ( $405.81 \mathrm{mbsf}$ ) is significantly higher than those of the silty clay samples, which reflects the lithology difference.

The preconsolidation pressure, $P^{\prime}{ }_{c}$, is determined using the work-stress method proposed by Becker et al. (1987). Figure F3 illustrates this approach for one sample (CRS802). $P^{\prime}{ }_{c}$ is significantly less than the hydrostatic vertical effective stress $\left(\sigma^{\prime}{ }_{v h}\right)$ at both sites (Fig. F39), which suggests that Ursa sediments are overpressured. 


\section{Acknowledgments}

This research used samples provided by the Integrated Ocean Drilling Program (IODP). Funding for this research was provided by the U.S. Science Support Program for IODP that is administered by Joint Oceanographic Institutions (now renamed Consortium for Ocean Leadership). In addition, U.S. National Science Foundation Grants 0447235 and 0351085 supported this research. This research was also sponsored by the Petroleum Research Fund (PRF\# 44476-AC8).

\section{References}

ASTM International, 2006. Standard test method for onedimensional consolidation properties of saturated cohesive soils using controlled-strain loading (Standard D4186-06). In Annual Book of ASTM Standards (Vol. 04.08): Soil and Rock (I): West Conshohocken, PA (Am. Soc. Testing and Mater.)
Becker, D.E., Crooks, J.H.A., Been, K., and Jeffries, M.G., 1987. Work as a criterion for determining in situ and yield stresses in clays. Can. Geotech. J., 24(4):549-564.

Casagrande, A., 1936. The determination of pre-consolidation load and its practical significance. In Casagrande, A., Rutledge, P.C., and Watson, J.D. (Eds.), Proc. 1st Int. Conf. Soil Mech. Found. Eng. Am. Soc. Civ. Eng., 3:60-64.

Graber, K.K., Pollard, E., Jonasson, B., and Schulte, E. (Eds.), 2002. Overview of Ocean Drilling Program Engineering Tools and Hardware. ODP Tech. Note, 31. doi:10.2973/odp.tn.31.2002

Tan, B., Germaine, J.T., and Flemings, P.B., 2006. Data report: consolidation and strength characteristics of sediments from ODP Site 1244, Hydrate Ridge, Cascadia continental margin. In Tréhu, A.M., Bohrmann, G., Torres, M.E., and Colwell, F.S. (Eds.), Proc. ODP, Sci. Results, 204: College Station, TX (Ocean Drilling Program), 1-148. doi:10.2973/odp.proc.sr.204.102.2006

Initial receipt: 22 June 2007

Acceptance: 31 January 2008

Publication: 1 July 2008

MS 308-204 
Figure F1. A. IODP Expedition 308 site locations (red circles) and bathymetry contours. Ursa Basin is located $210 \mathrm{~km}$ southeast of New Orleans, Louisiana, USA (inset map). Contour interval $=100 \mathrm{~m}$. B. East-west seismic cross section A-A' (located in A). VE $=$ vertical exaggeration. C. Interpreted cross section $\mathrm{A}-\mathrm{A}^{\prime}$. Light and dark gray = mud-rich levee, rotated channel-margin slides, and hemipelagic drape; yellow = sand-rich channel fill. Blue Unit (light blue) composed of sand and mud. Mass transport deposits (MTDs) have occurred in the mud-rich levee deposits above the Blue Unit. Red = detachment surfaces.
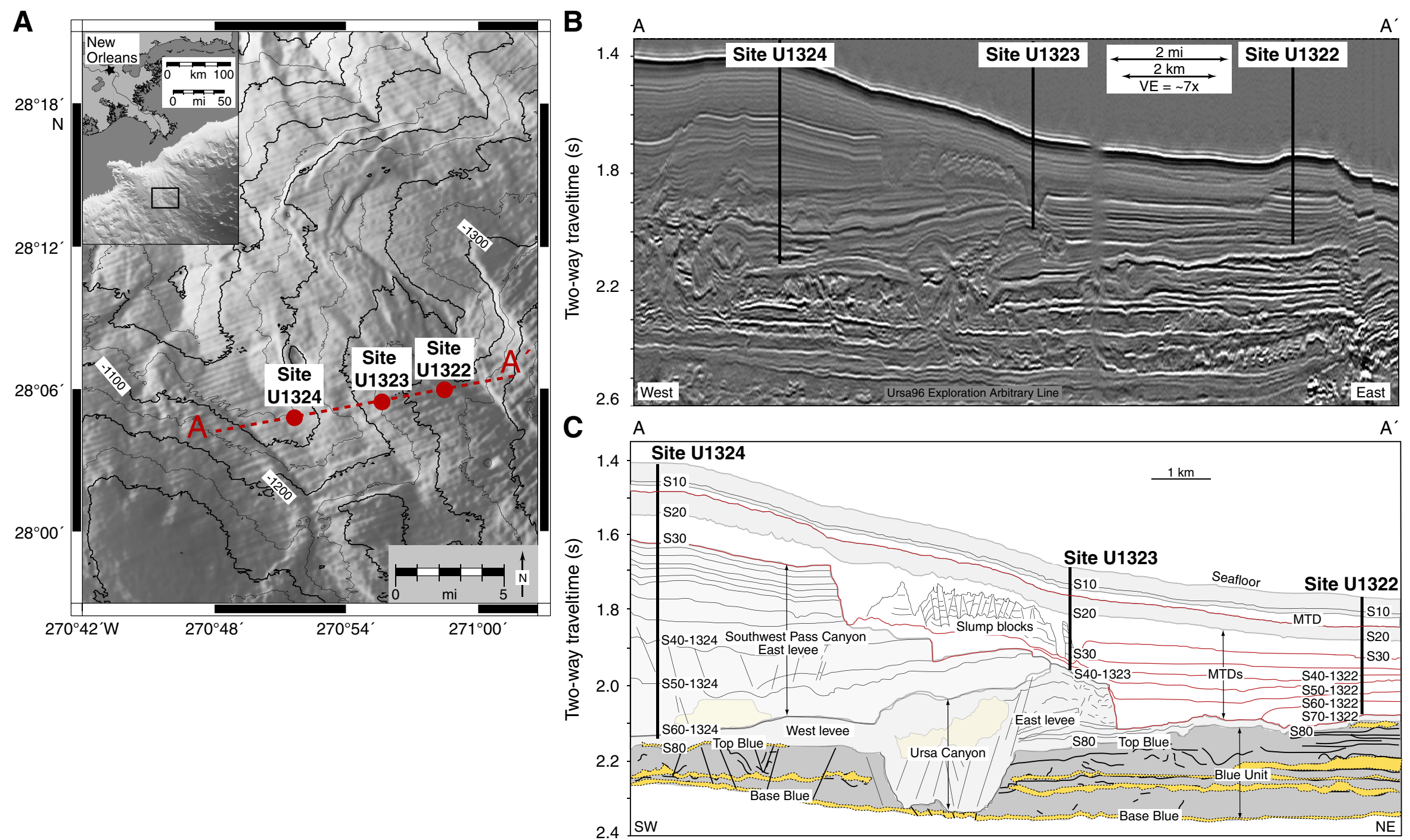
Figure F2. Examples of consolidation test results. $\sigma_{v \mathrm{vh}}^{\prime}=$ hydrostatic vertical effective stress calculated from LWD bulk density profile and assumed seawater density of $1.024 \mathrm{~g} / \mathrm{cm}^{3}$. Preconsolidation pressures ( $P^{\prime}{ }_{c}$, open circle) derived using work-stress method (Becker et al., 1987).

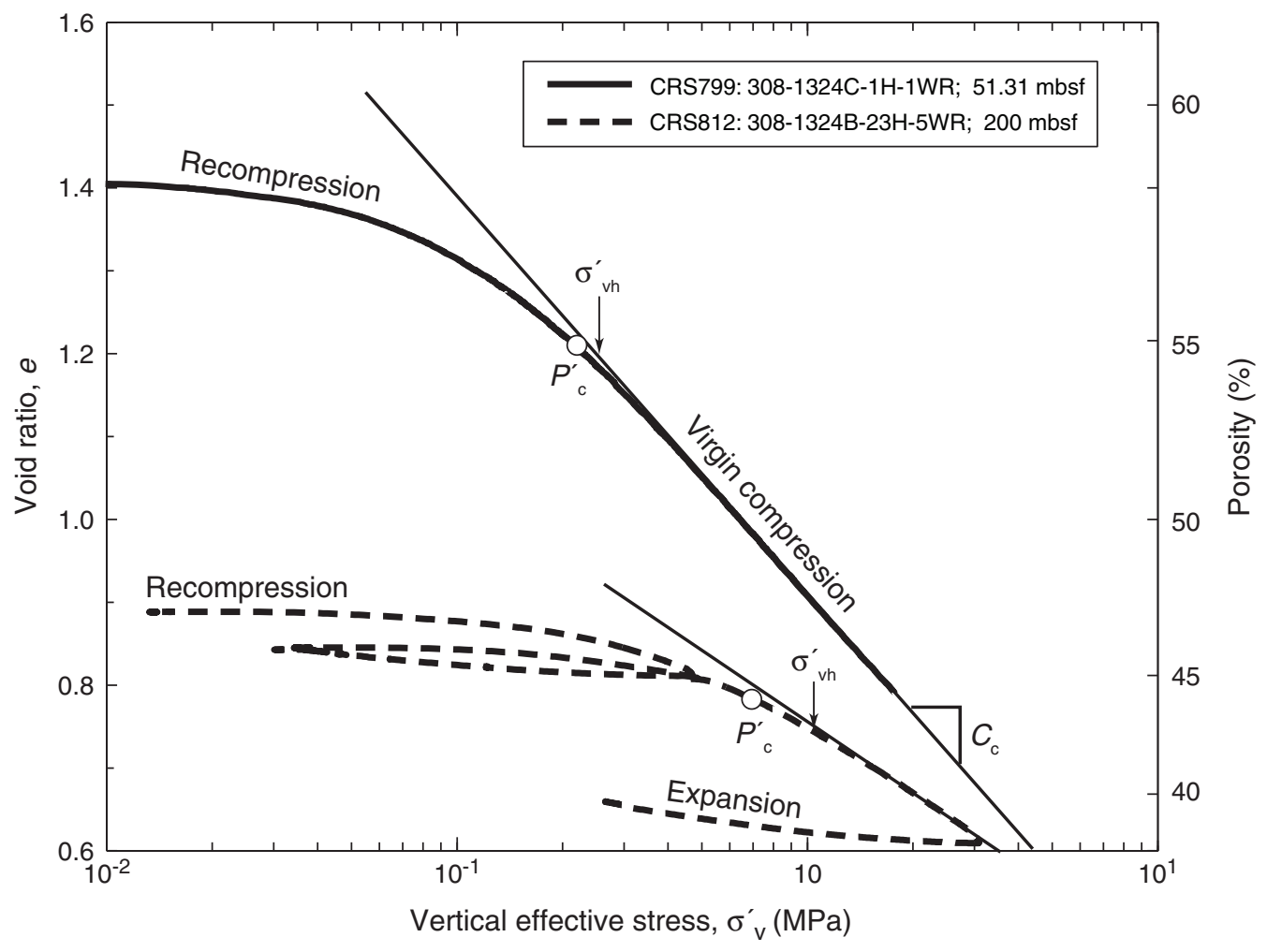


Figure F3. Derivation of preconsolidation pressure using work-stress method of Becker et al. (1987) based on data from CRS802.

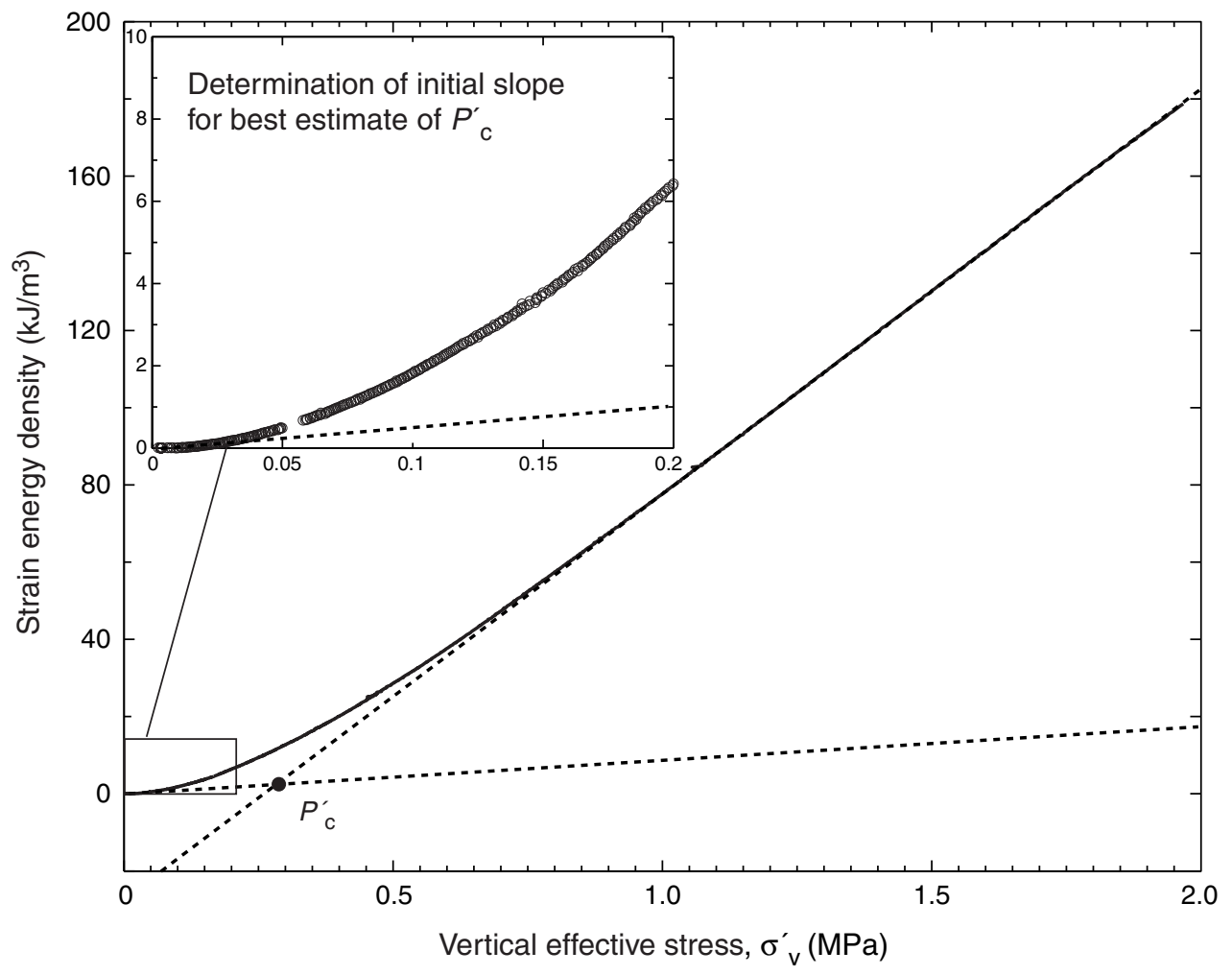


Figure F4. CRS796 consolidation data for Sample 308-U1322D-2H-2WR, 72.78 mbsf. Coef. = coefficient.

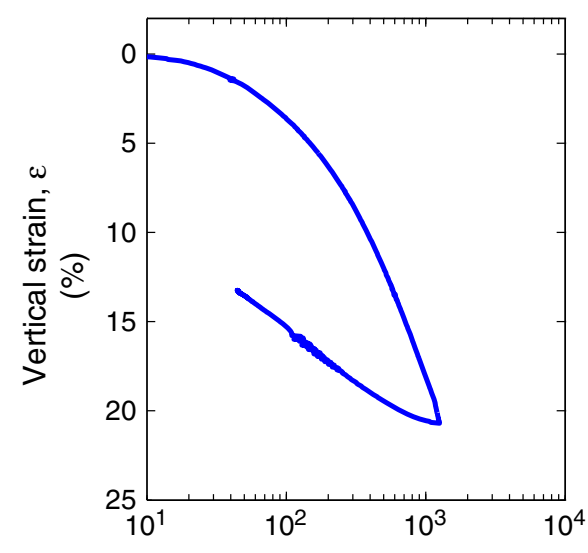

Vertical consolidation stress, $\sigma_{v}^{\prime}$ ( $\mathrm{kPa})$

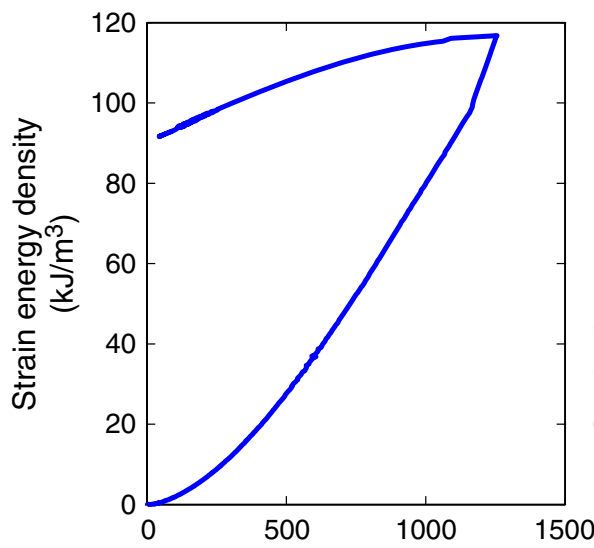

Vertical consolidation stress, $\sigma_{v}^{\prime}$ (kPa)

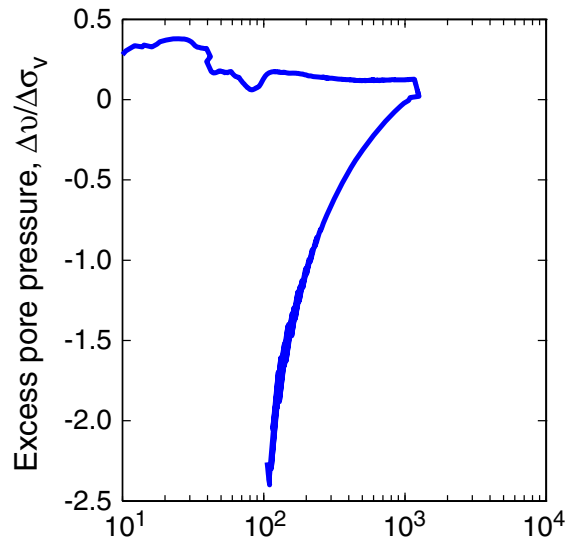

Vertical consolidation stress, $\sigma_{\mathrm{v}}^{\prime}$ $(\mathrm{kPa})$

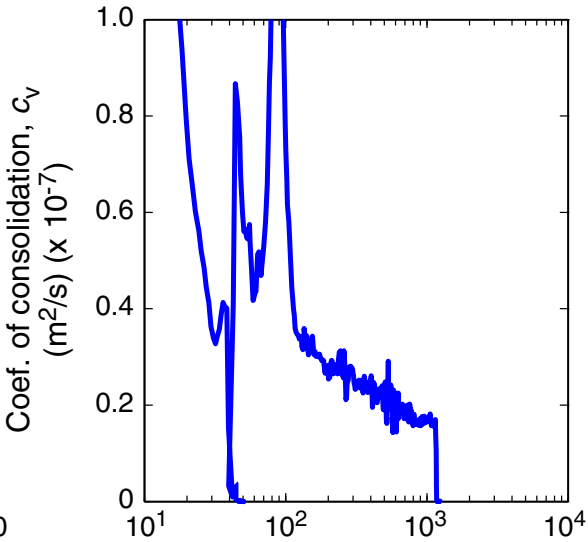

Vertical consolidation stress, $\sigma_{v}^{\prime}$ (kPa)

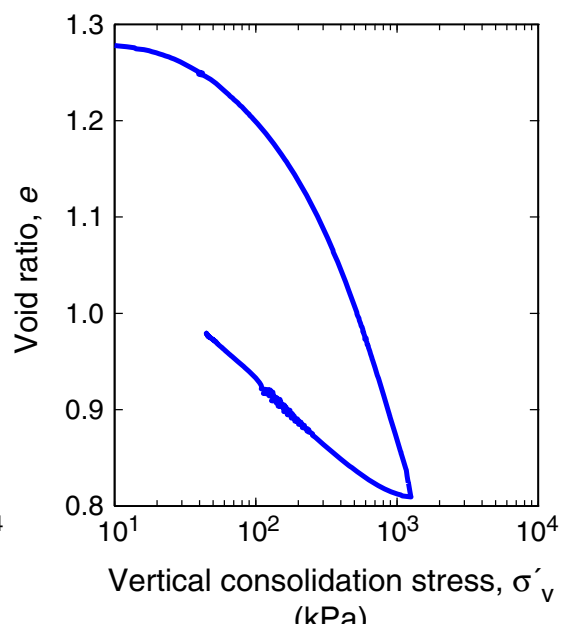

(kPa)

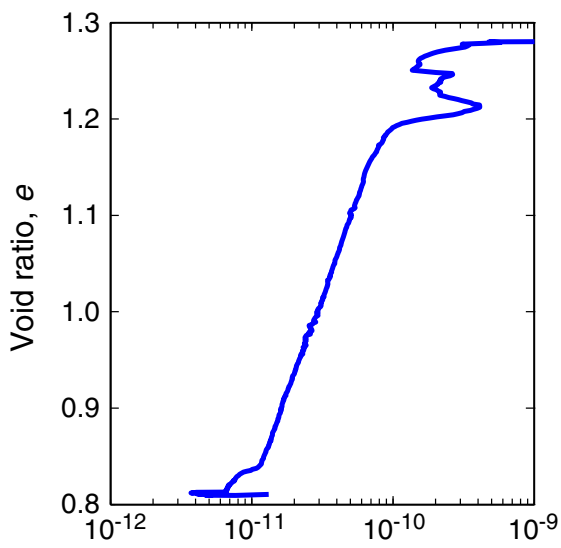

Hydraulic conductivity, $K$ $(\mathrm{m} / \mathrm{s})$ 
Figure F5. CRS797 consolidation data for Sample 308-U1324C-1H-1WR, 51.27 mbsf. Coef. = coefficient.

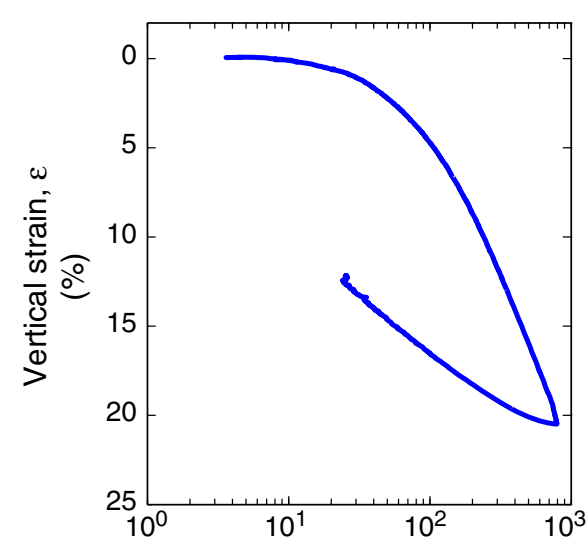

Vertical consolidation stress, $\sigma_{v}^{\prime}$ ( $\mathrm{kPa}$ )

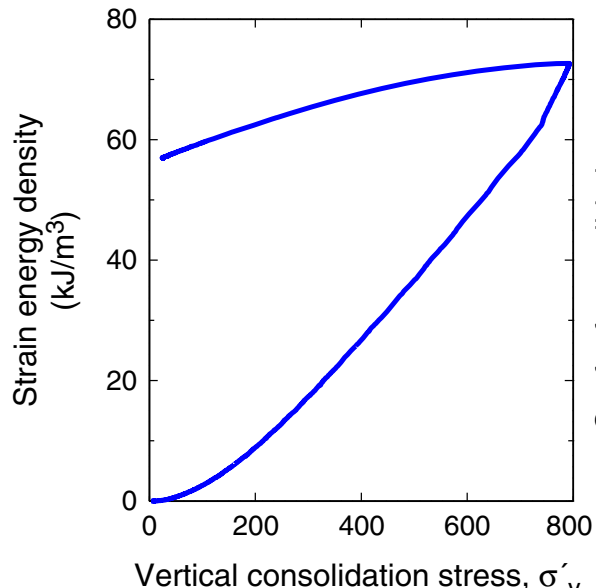

$(\mathrm{kPa})$

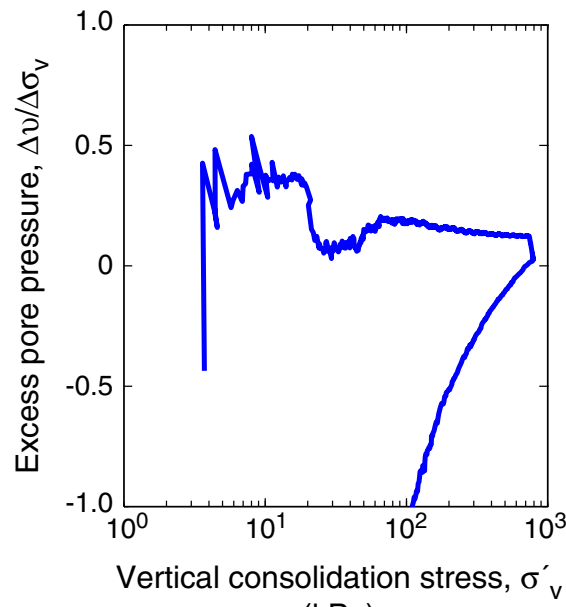

(kPa)

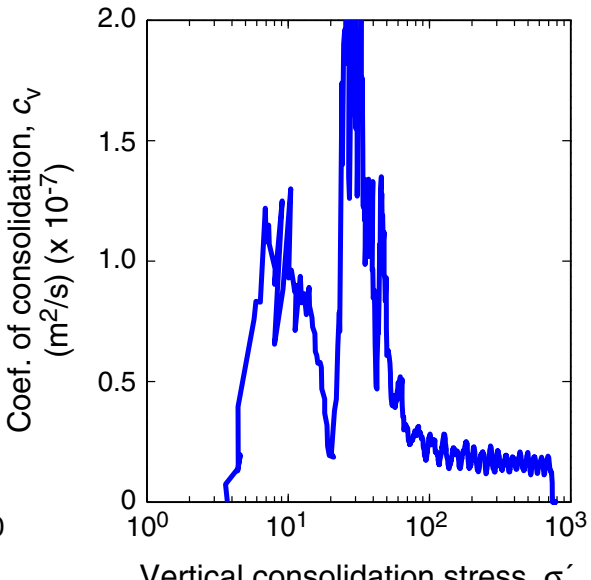

Vertical consolidation stress, $\sigma_{\mathrm{v}}^{\prime}$ (kPa)

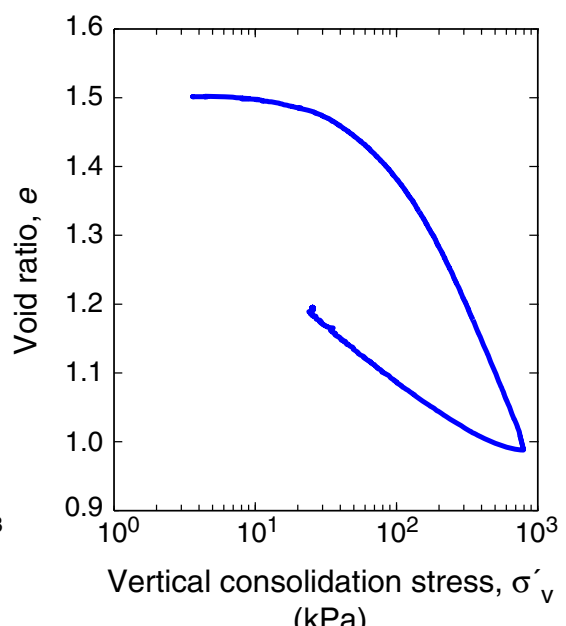

(kPa)

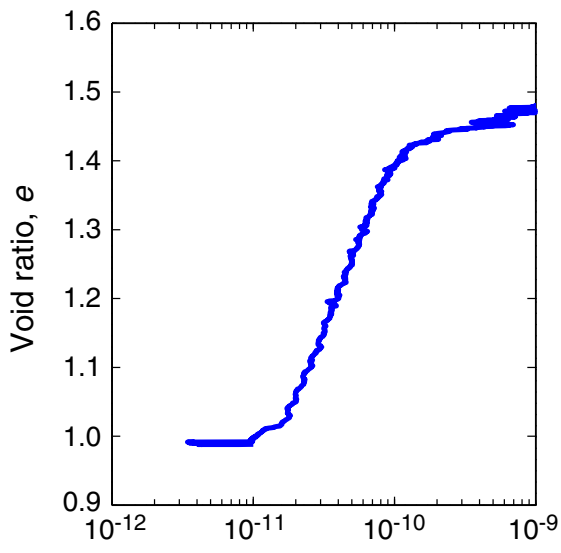

Hydraulic conductivity, $K$ $(\mathrm{m} / \mathrm{s})$ 
Figure F6. CRS798 consolidation data for Sample 308-U1322D-2H-2WR, 72.83 mbsf. Coef. = coefficient.

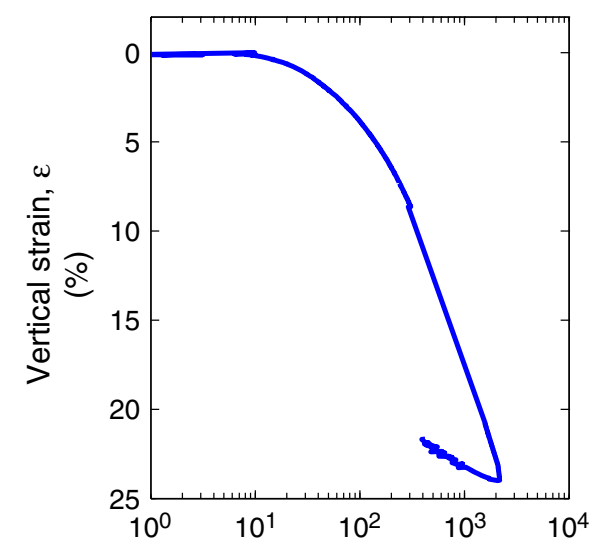

Vertical consolidation stress, $\sigma_{\text {v }}^{\prime}$ (kPa)

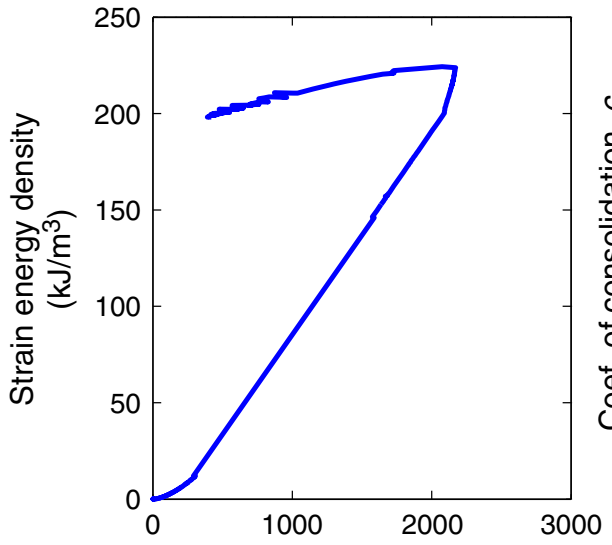

Vertical consolidation stress, $\sigma_{\mathrm{v}}^{\prime}$ $(\mathrm{kPa})$

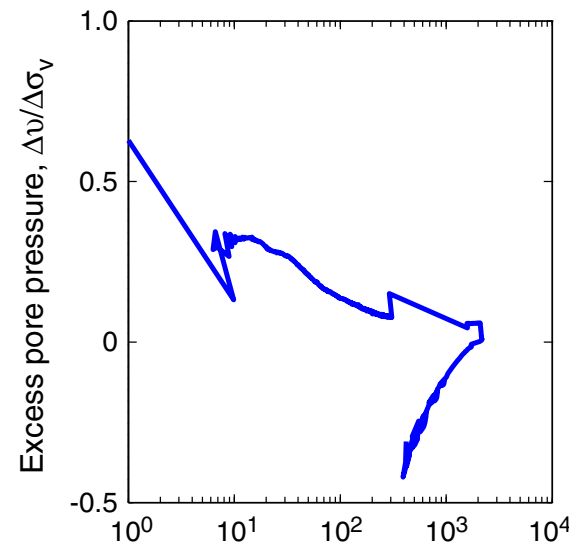

Vertical consolidation stress, $\sigma_{\text {v }}^{\prime}$ $(\mathrm{kPa})$

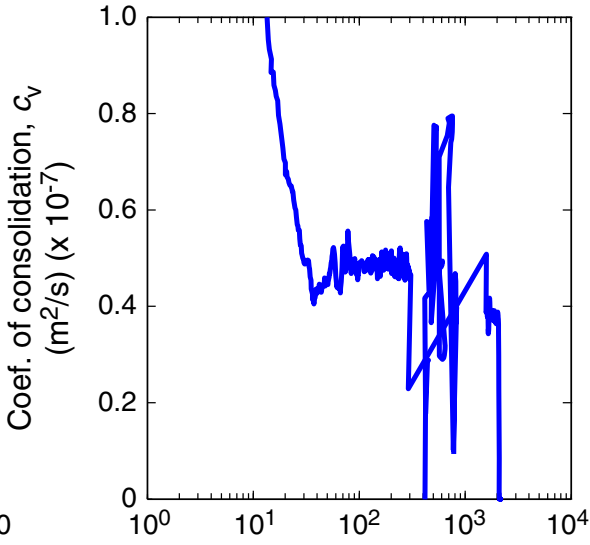

Vertical consolidation stress, $\sigma_{\mathrm{v}}^{\prime}$ (kPa)

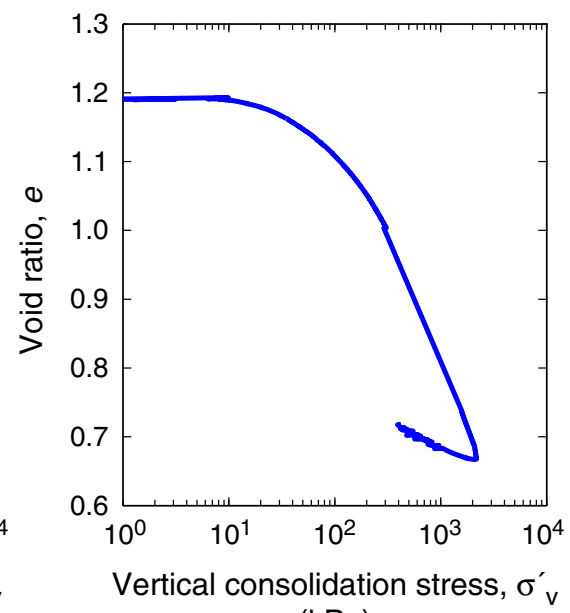

(kPa)

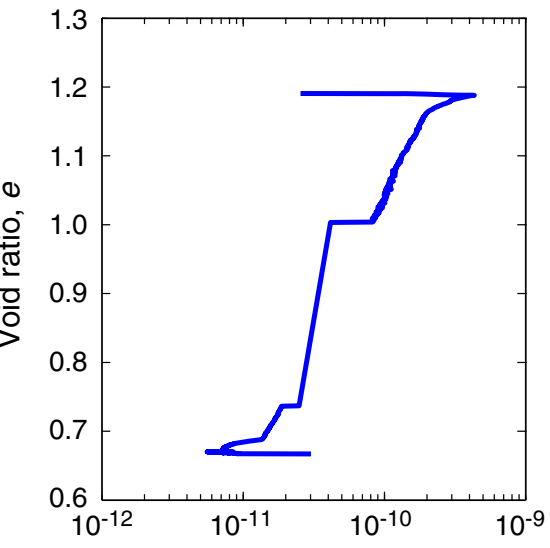

Hydraulic conductivity, $K$ $(\mathrm{m} / \mathrm{s})$ 
Figure F7. CRS799 consolidation data for Sample 308-U1324C-1H-1WR, 51.31 mbsf. Coef. = coefficient.

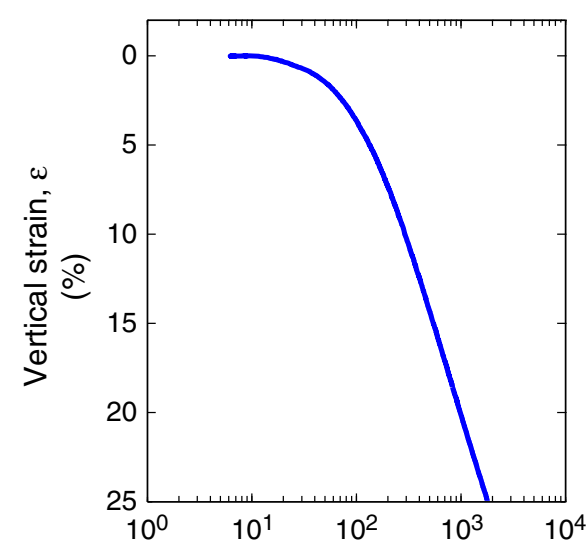

Vertical consolidation stress, $\sigma^{\prime}$ v ( $\mathrm{kPa}$ )

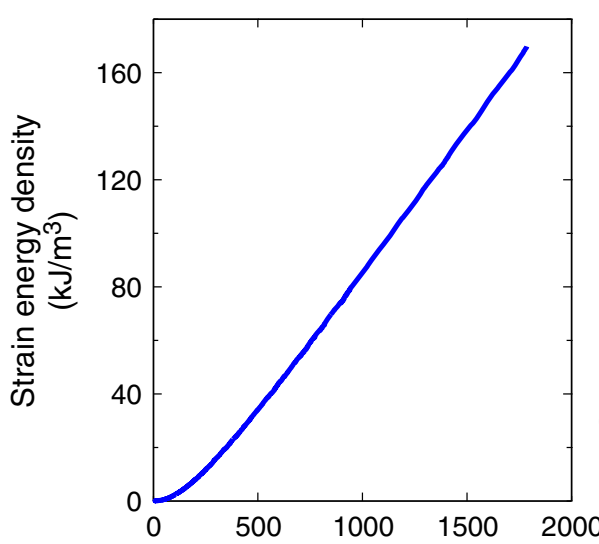

Vertical consolidation stress, $\sigma_{\mathrm{v}}^{\prime}$ $(\mathrm{kPa})$

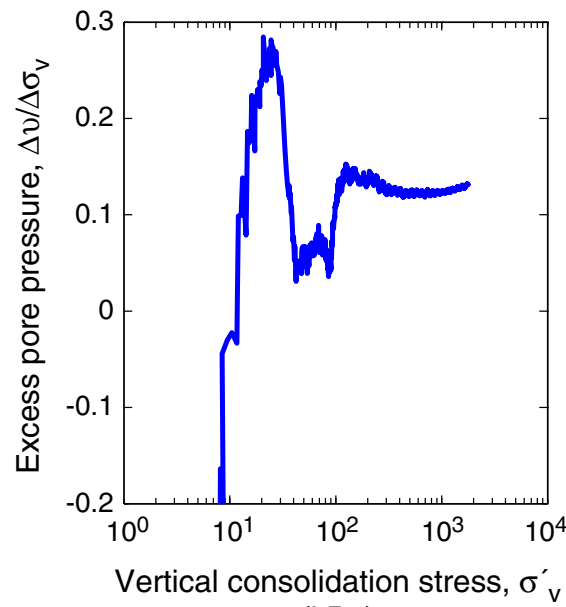

(kPa)

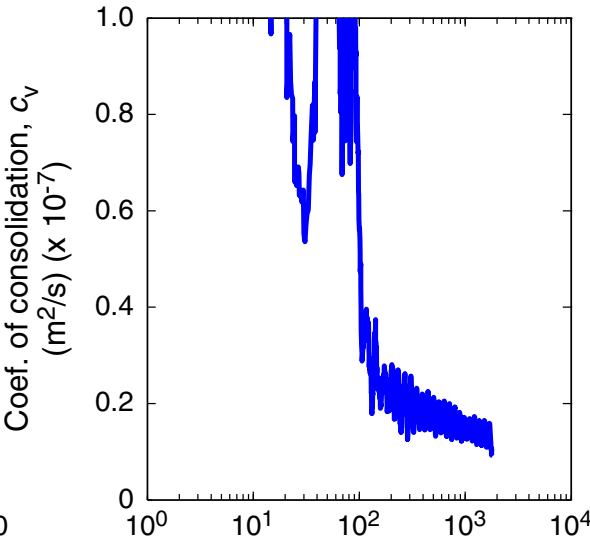

Vertical consolidation stress, $\sigma_{\mathrm{v}}^{\prime}$ (kPa)

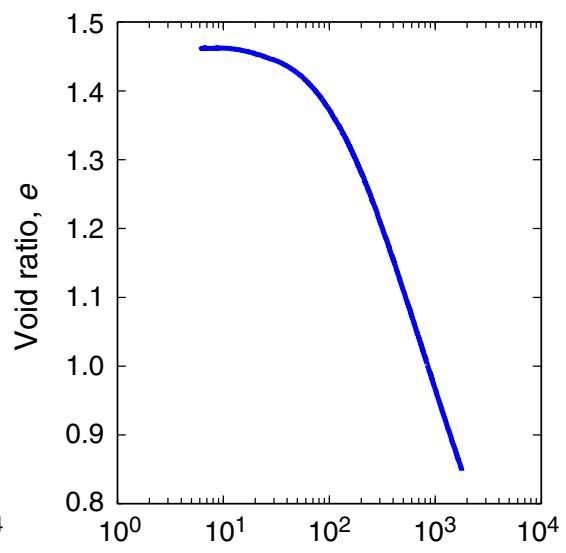

Vertical consolidation stress, $\sigma_{v}^{\prime}$ (kPa)

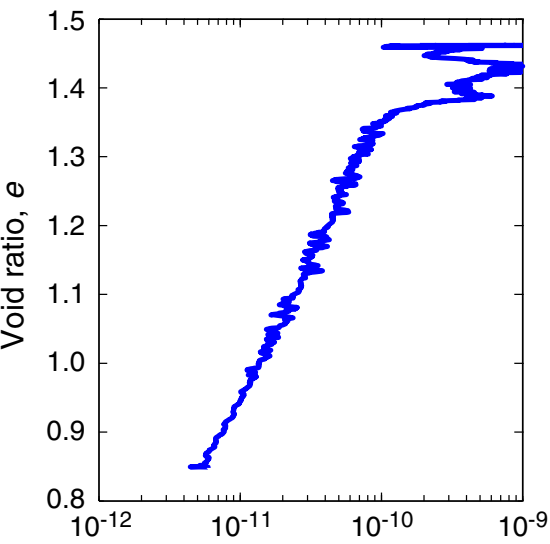

Hydraulic conductivity, $K$ $(\mathrm{m} / \mathrm{s})$ 
Figure F8. CRS800 consolidation data for Sample 308-U1324B-4H-7WR, 31.86 mbsf. Coef. = coefficient.

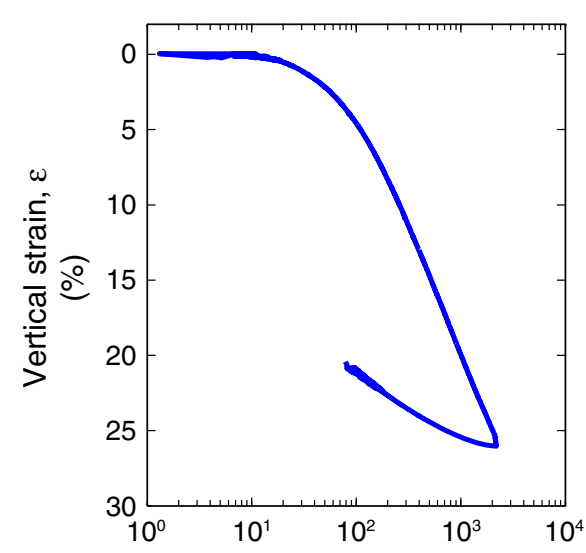

Vertical consolidation stress, $\sigma^{\prime}$, (kPa)

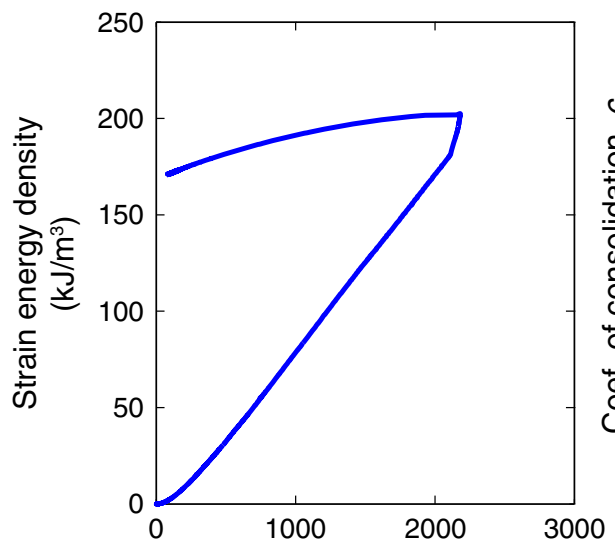

Vertical consolidation stress, $\sigma^{\prime}$ (kPa)

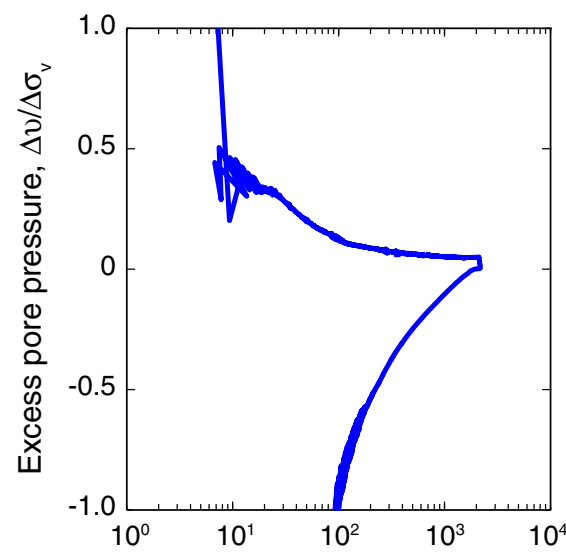

Vertical consolidation stress, $\sigma^{\prime}$ (kPa)

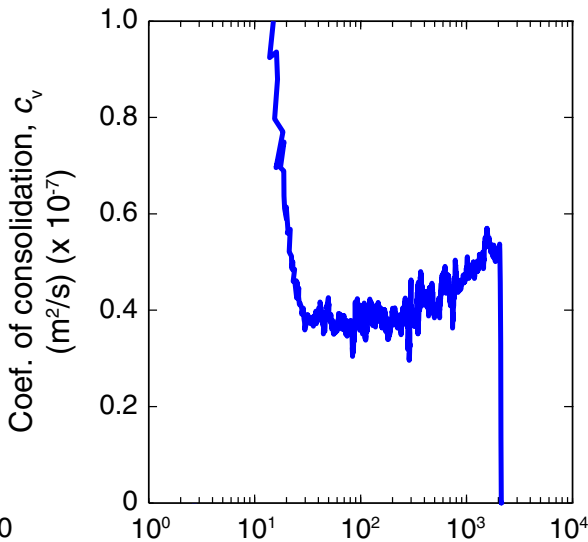

Vertical consolidation stress, $\sigma_{\text {v }}^{\prime}$ ( $\mathrm{kPa})$

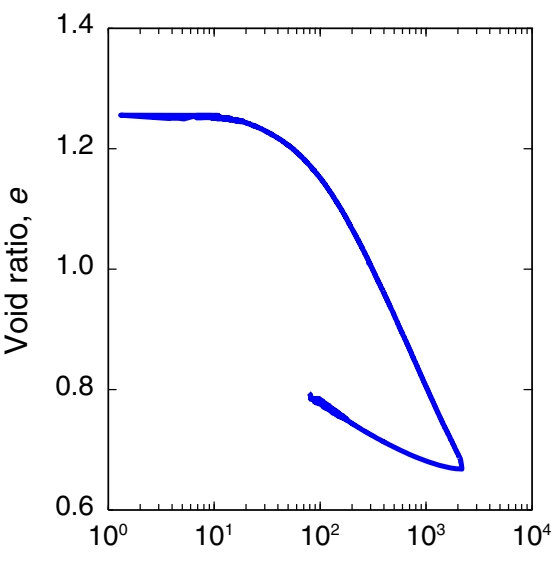

Vertical consolidation stress, $\sigma^{\prime}$ (kPa)

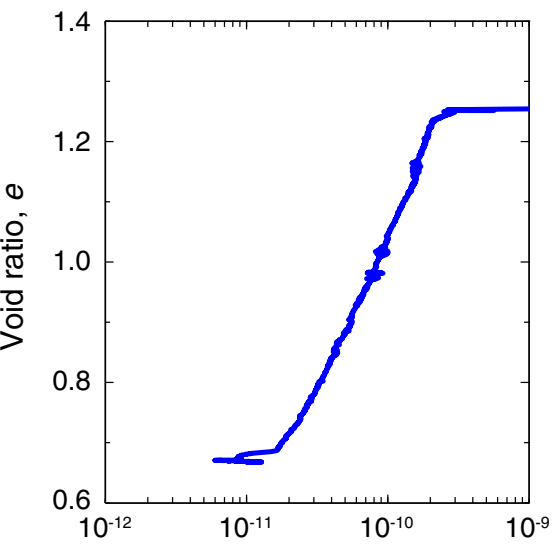

Hydraulic conductivity, $K$ $(\mathrm{m} / \mathrm{s})$ 
Figure F9. CRS801 consolidation data for Sample 308-U1324B-16H-5WR, 142.13 mbsf. Coef. = coefficient.w

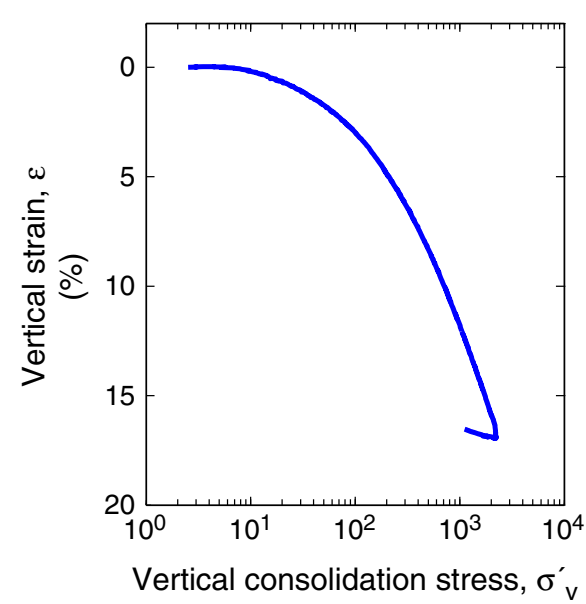

$(\mathrm{kPa})$

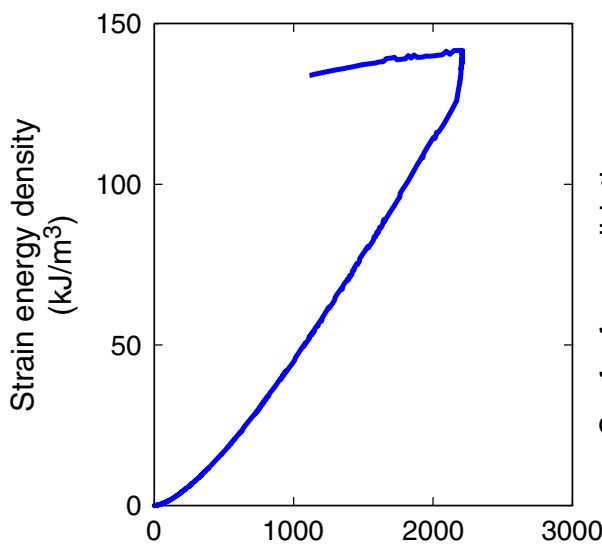

Vertical consolidation stress, $\sigma^{\prime}$ v $(\mathrm{kPa})$

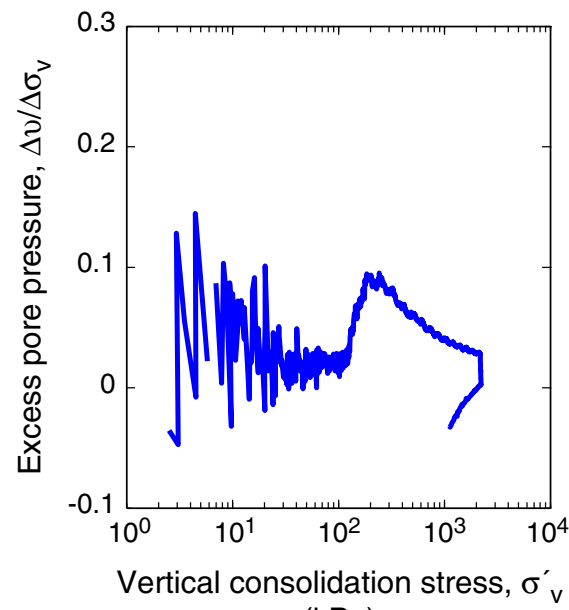

(kPa)

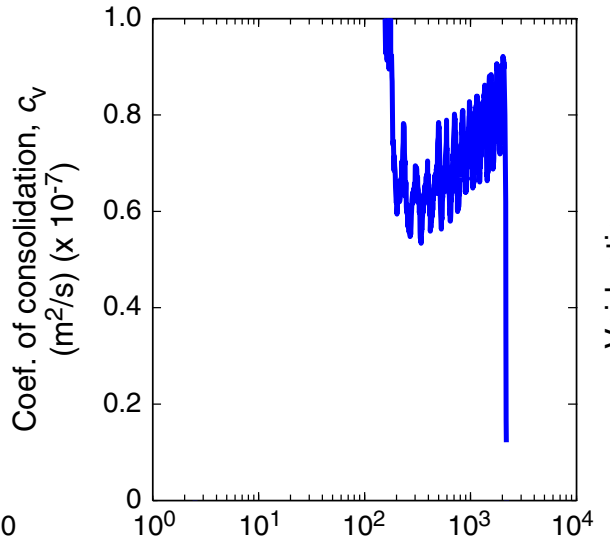

Vertical consolidation stress, $\sigma_{\text {v }}^{\prime}$ (kPa)

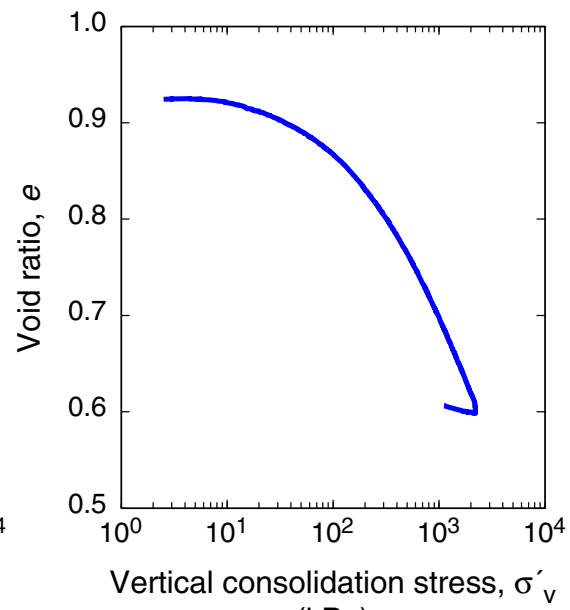

(kPa)

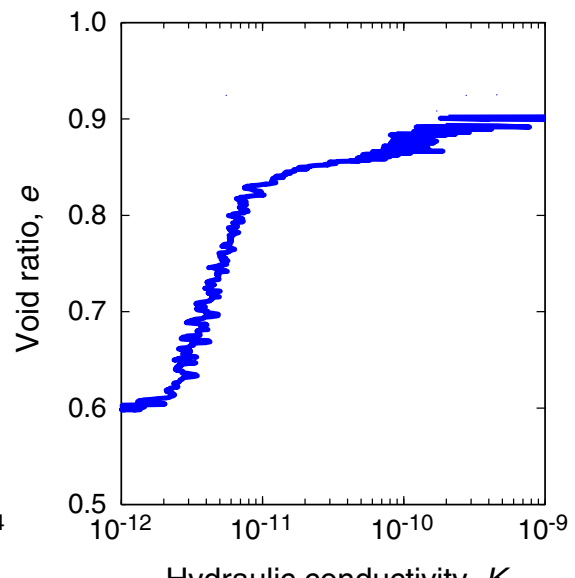

Hydraulic conductivity, $K$ $(\mathrm{m} / \mathrm{s})$ 
Figure F10. CRS802 consolidation data for Sample 308-U1324B-7H-7WR, 60.31 mbsf. Coef. = coefficient.

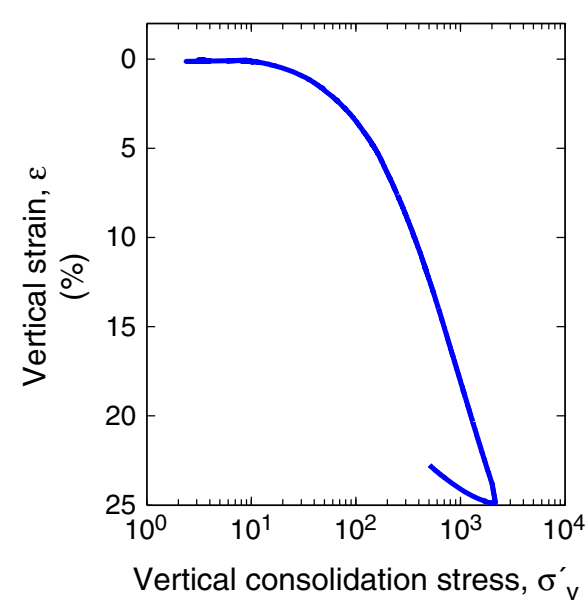

$(\mathrm{kPa})$

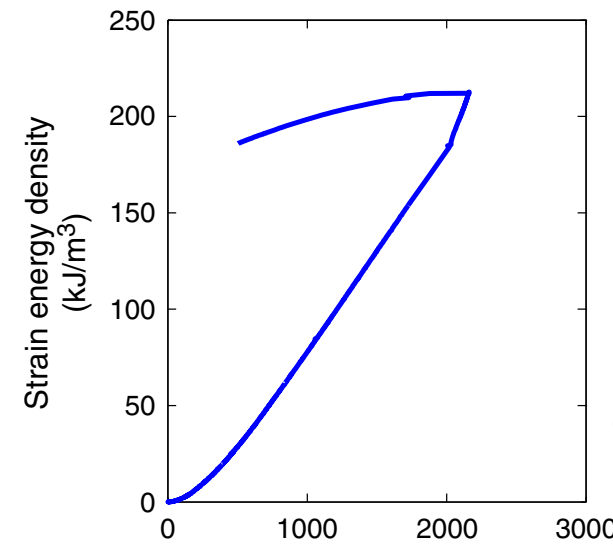

Vertical consolidation stress, $\sigma_{\text {v }}^{\prime}$ $(\mathrm{kPa})$

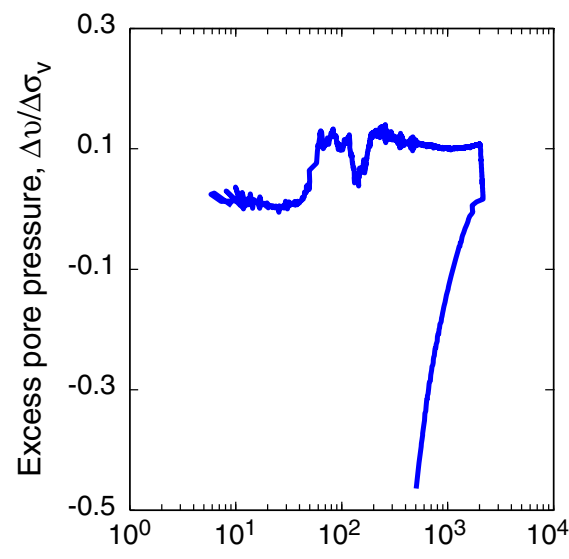

Vertical consolidation stress, $\sigma_{v}^{\prime}$ (kPa)

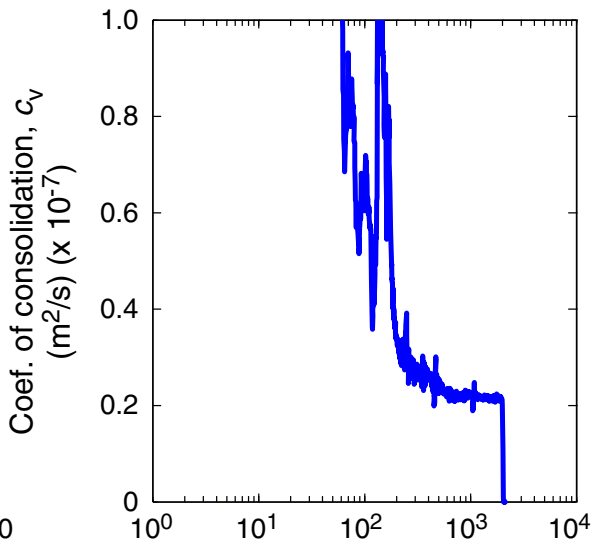

Vertical consolidation stress, $\sigma_{\mathrm{v}}^{\prime}$ (kPa)

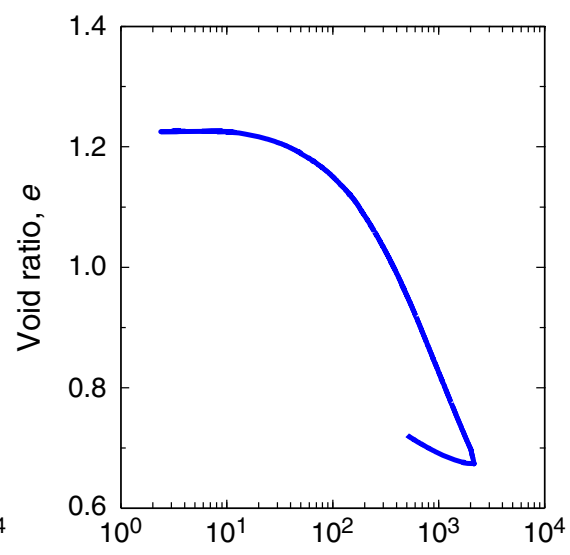

Vertical consolidation stress, $\sigma_{\mathrm{v}}^{\prime}$ (kPa)

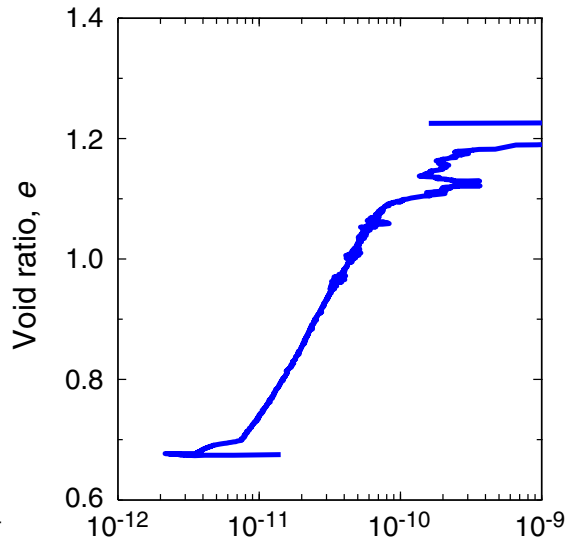

Hydraulic conductivity, $K$ $(\mathrm{m} / \mathrm{s})$ 
Figure F11. CRS803 consolidation data for Sample 308-U1324B-15H-5WR, 134.2 mbsf. Coef. = coefficient.

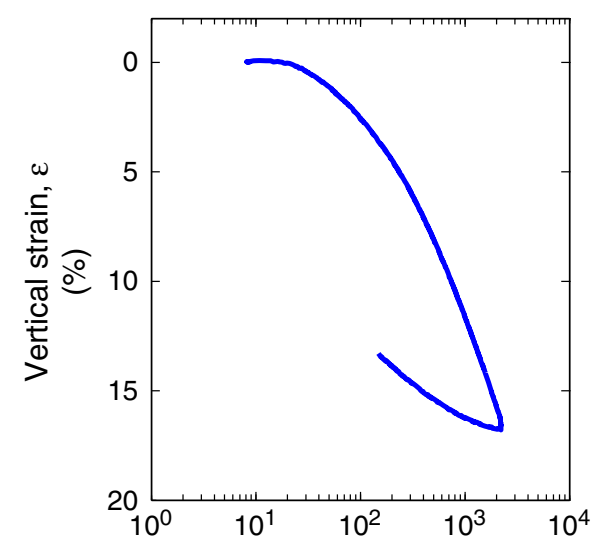

Vertical consolidation stress, $\sigma^{\prime}$ v $(\mathrm{kPa})$

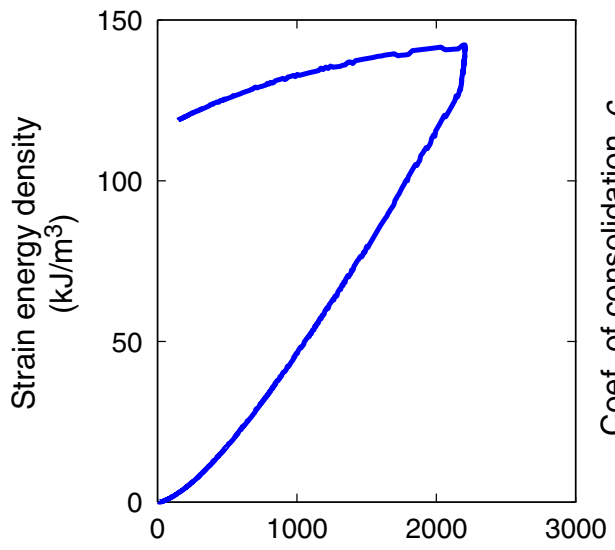

Vertical consolidation stress, $\sigma^{\prime}$ v

(kPa)

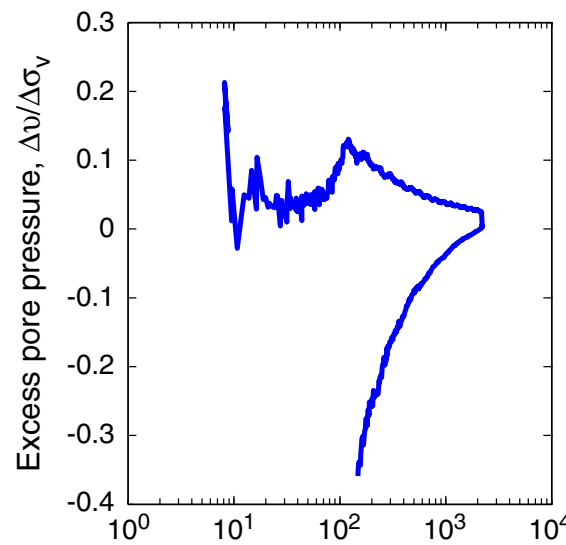

Vertical consolidation stress, $\sigma_{\mathrm{v}}^{\prime}$ (kPa)

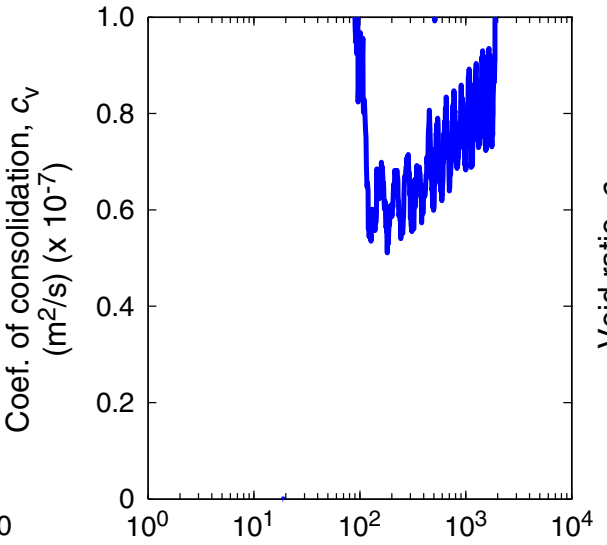

Vertical consolidation stress, $\sigma_{v}^{\prime}$ (kPa)

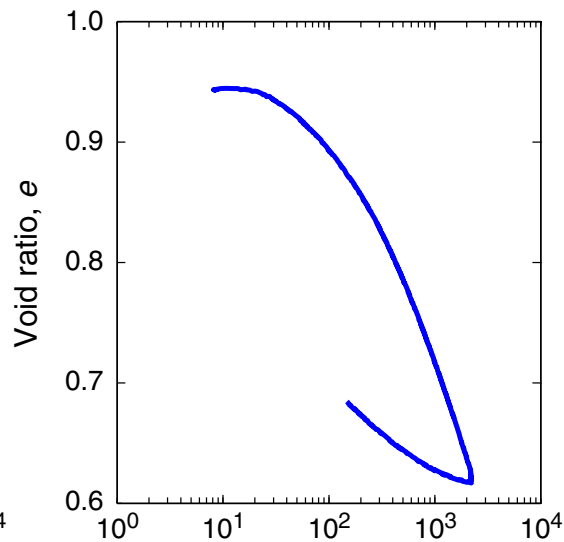

Vertical consolidation stress, $\sigma_{v}^{\prime}$ (kPa)

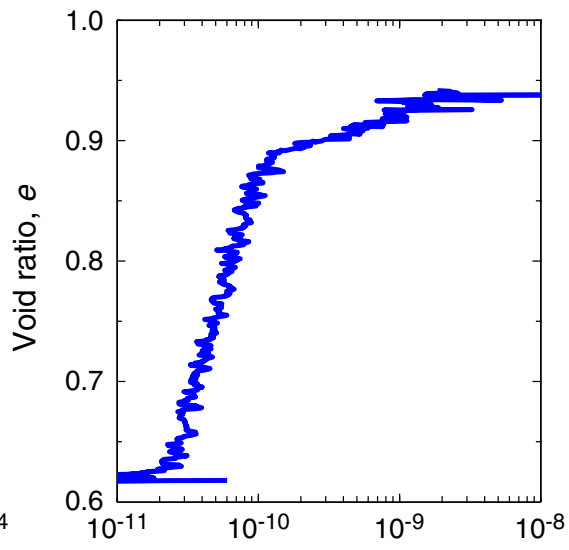

Hydraulic conductivity, $K$ $(\mathrm{m} / \mathrm{s})$ 
Figure F12. CRS807 consolidation data for Sample 308-U1324C-2H-4WR, 105.48 mbsf. Coef. = coefficient.

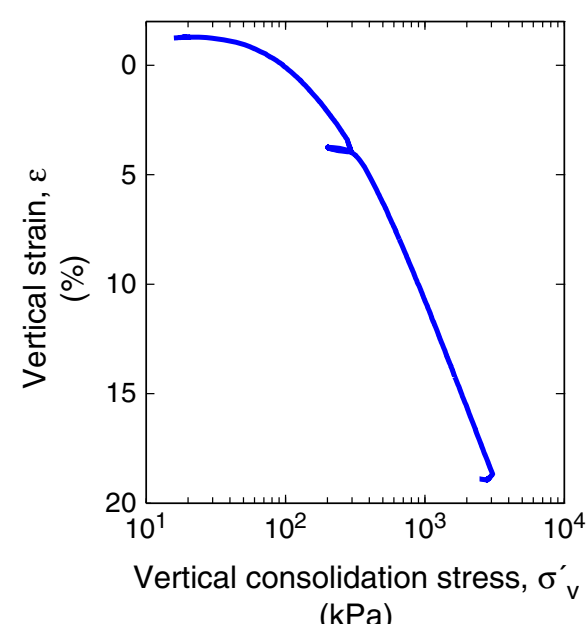

$(\mathrm{kPa})$

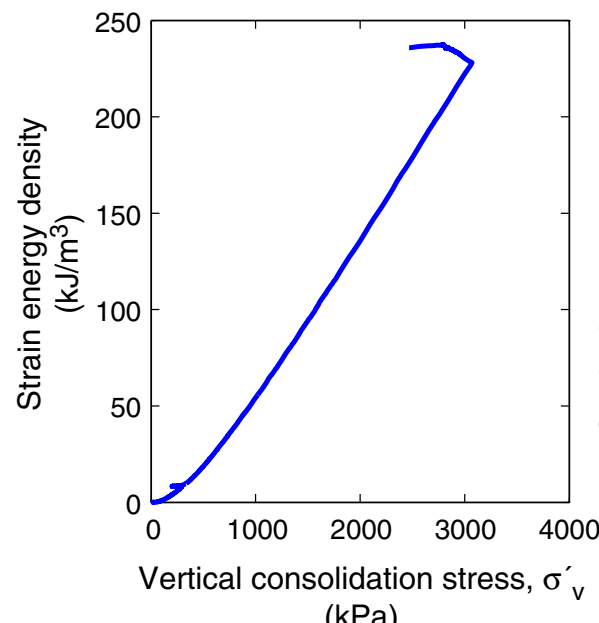

(kPa)

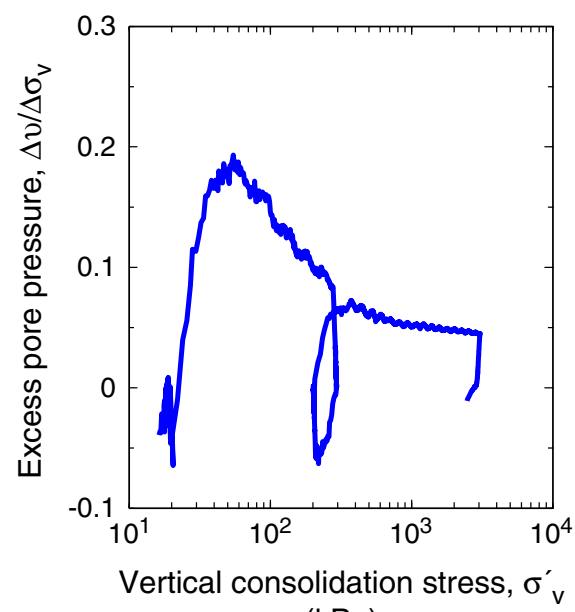

$(\mathrm{kPa})$

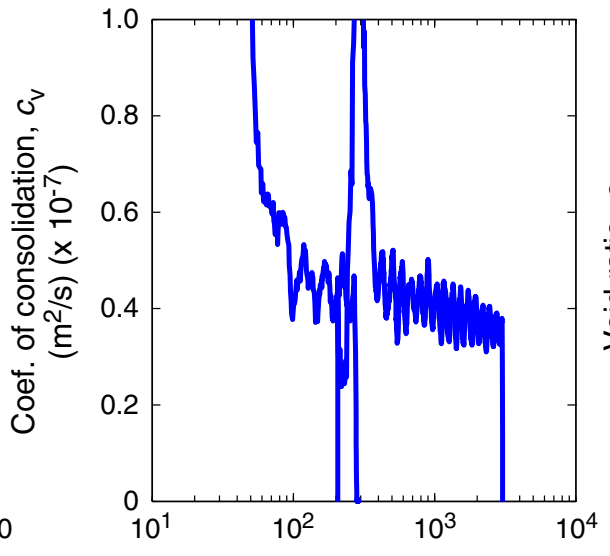

Vertical consolidation stress, $\sigma_{\mathrm{v}}^{\prime}$ (kPa)

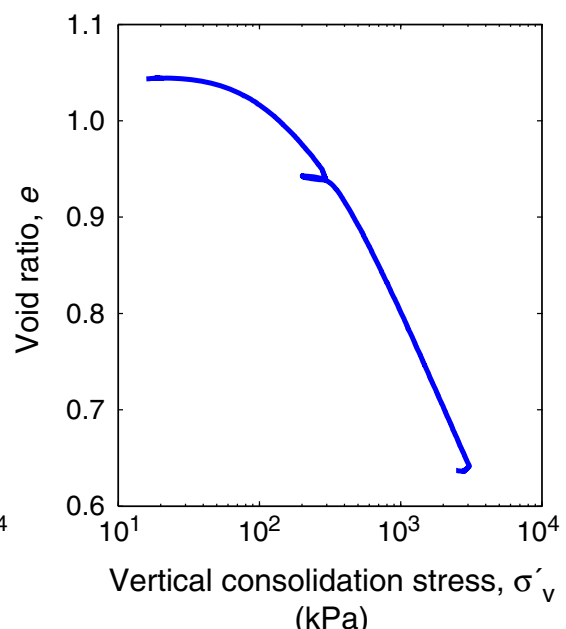

( $\mathrm{kPa}$ )

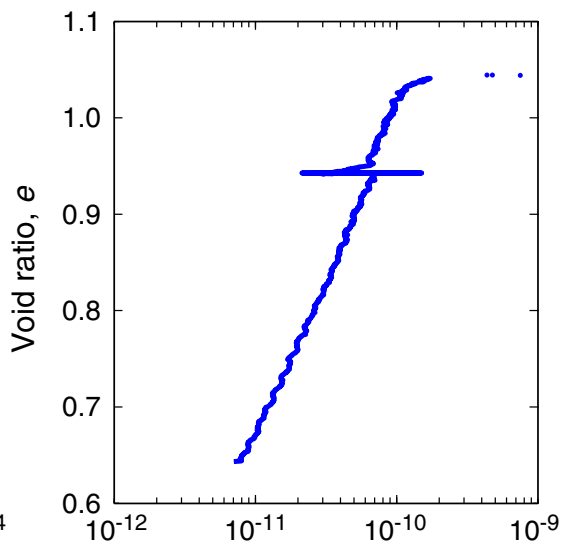

Hydraulic conductivity, $K$ $(\mathrm{m} / \mathrm{s})$ 
Figure F13. CRS808 consolidation data for Sample 308-U1322B-15H-1WR, 126.28 mbsf. Coef. = coefficient.

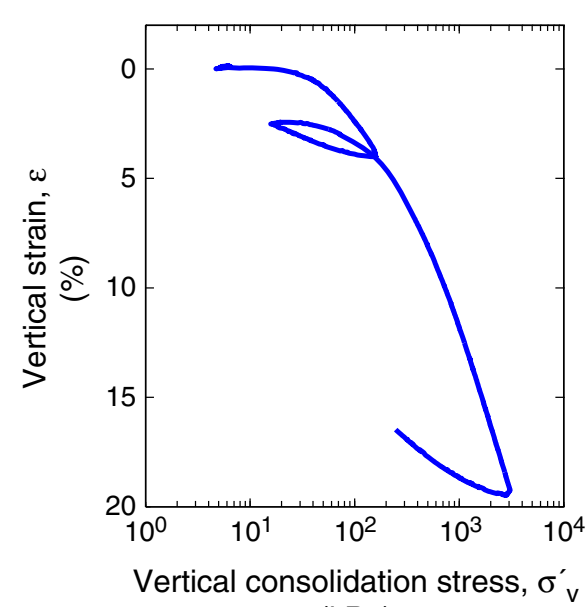

(kPa)

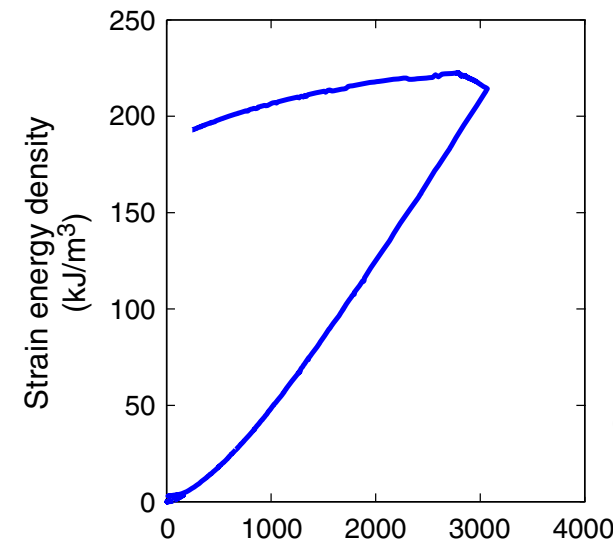

Vertical consolidation stress, $\sigma_{\text {v }}^{\prime}$ $(\mathrm{kPa})$

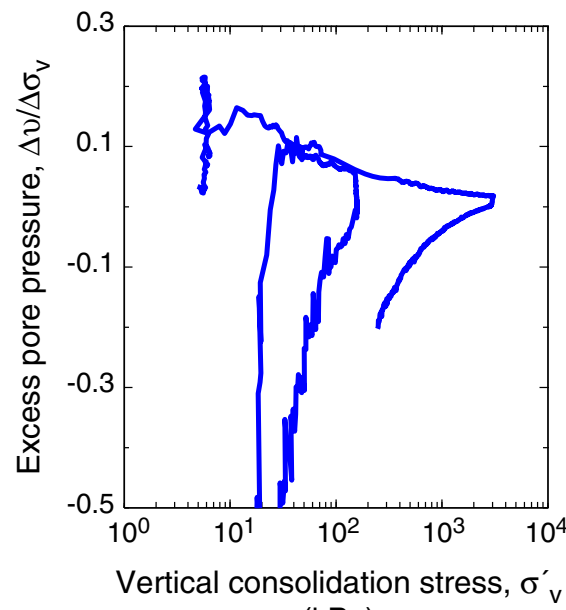

(kPa)

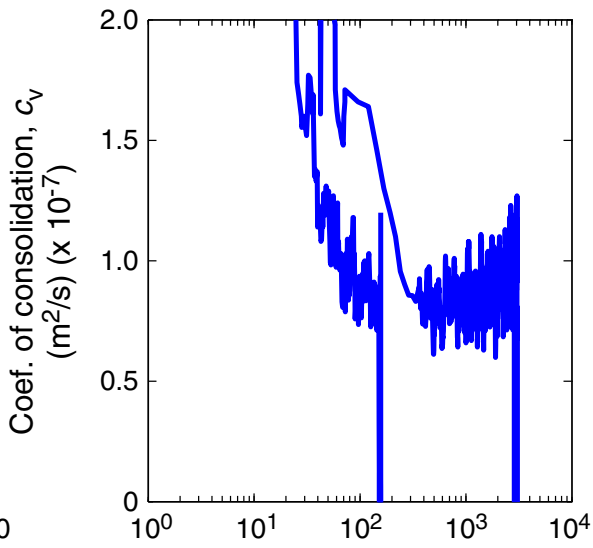

Vertical consolidation stress, $\sigma_{\text {v }}^{\prime}$ (kPa)

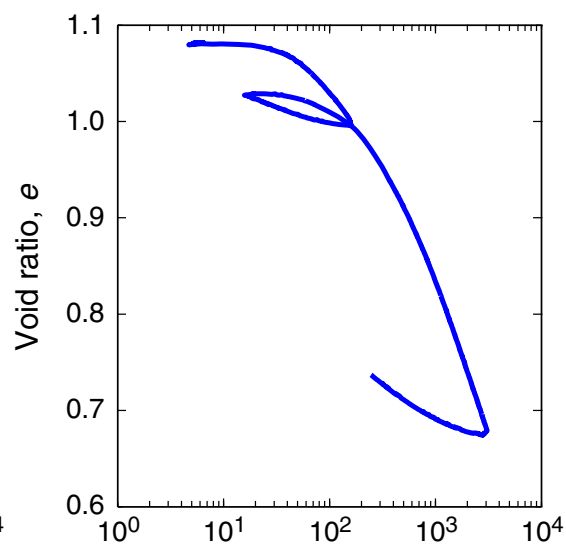

Vertical consolidation stress, $\sigma_{v}^{\prime}$ $(\mathrm{kPa})$

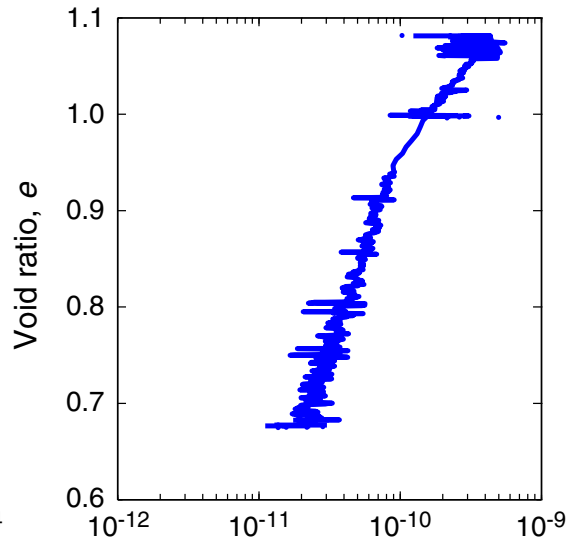

Hydraulic conductivity, $K$ $(\mathrm{m} / \mathrm{s})$ 
Figure F14. CRS810 consolidation data for Sample 308-U1322B-18H-6WR, 157.42 mbsf. Coef. = coefficient.
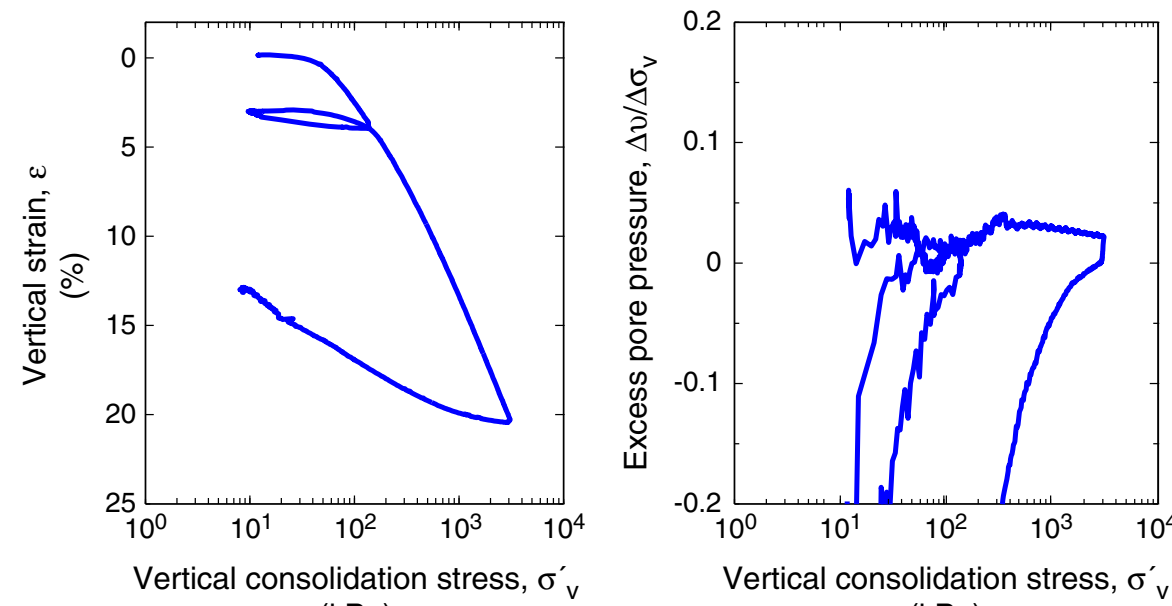

(kPa)

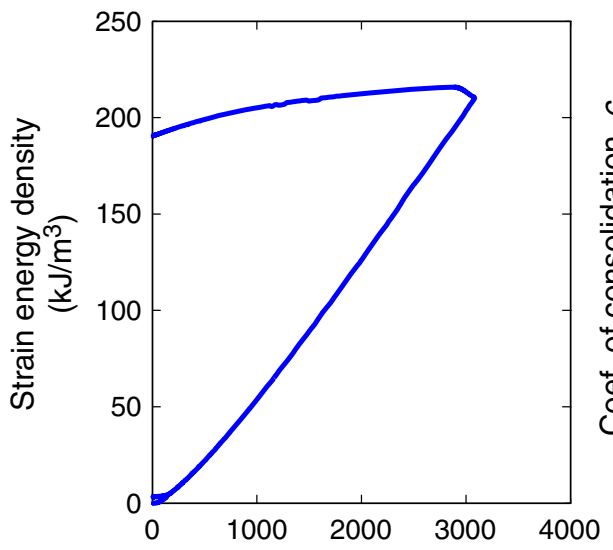

Vertical consolidation stress, $\sigma^{\prime}$ v

(kPa)

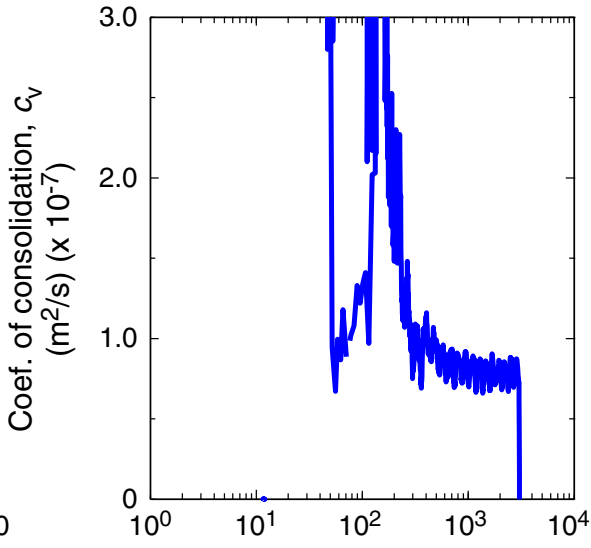

Vertical consolidation stress, $\sigma_{\text {v }}^{\prime}$ (kPa)

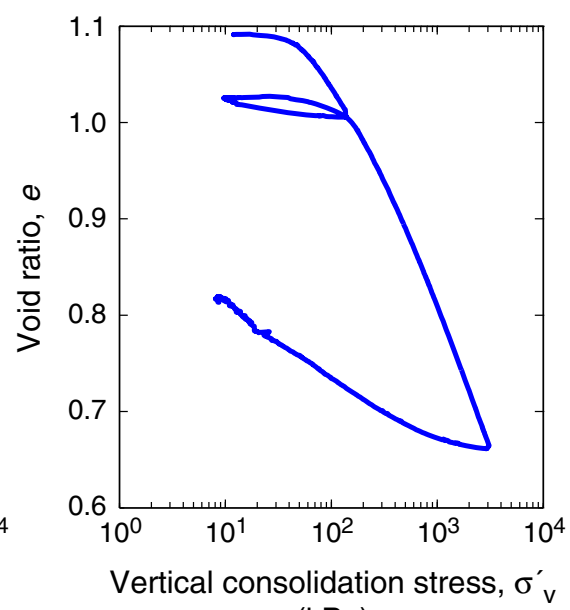

(kPa)

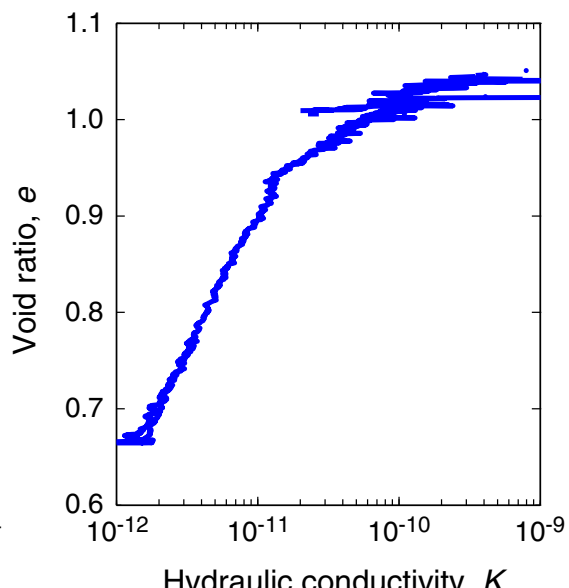

$(\mathrm{m} / \mathrm{s})$ 
Figure F15. CRS812 consolidation data for Sample 308-U1324B-23H-5WR, 200 mbsf. Coef. = coefficient.

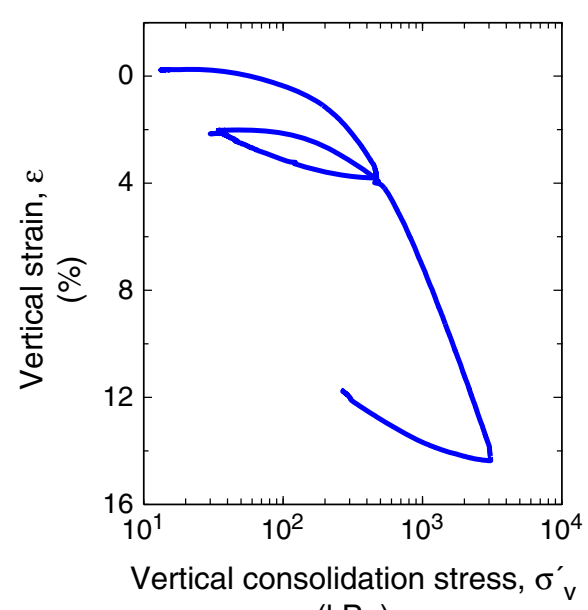

$(\mathrm{kPa})$

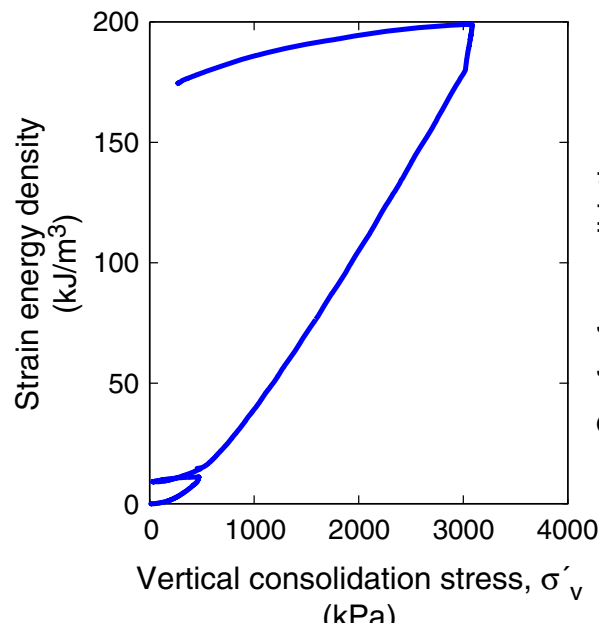

$(\mathrm{kPa})$

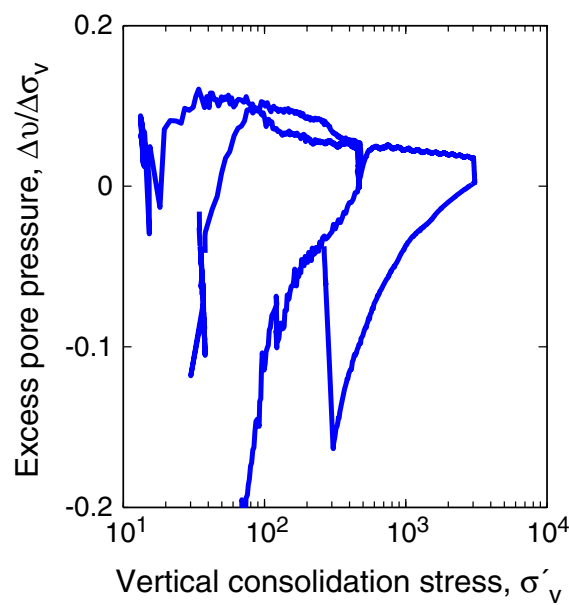

$(\mathrm{kPa})$

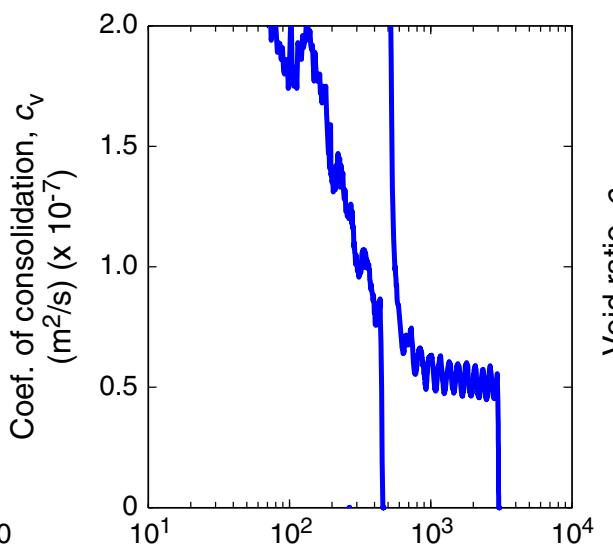

Vertical consolidation stress, $\sigma_{v}^{\prime}$ (kPa)

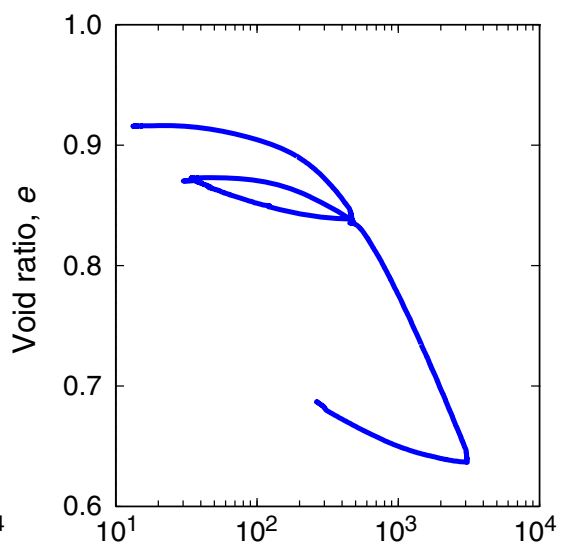

Vertical consolidation stress, $\sigma_{v}^{\prime}$ (kPa)

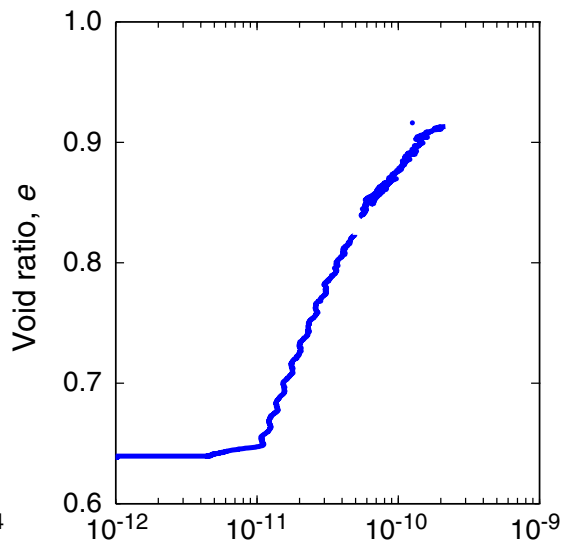

Hydraulic conductivity, $K$ $(\mathrm{m} / \mathrm{s})$ 
Figure F16. CRS813 consolidation data for Sample 308-U1324B-10H-7WR, 89.22 mbsf. Coef. = coefficient.
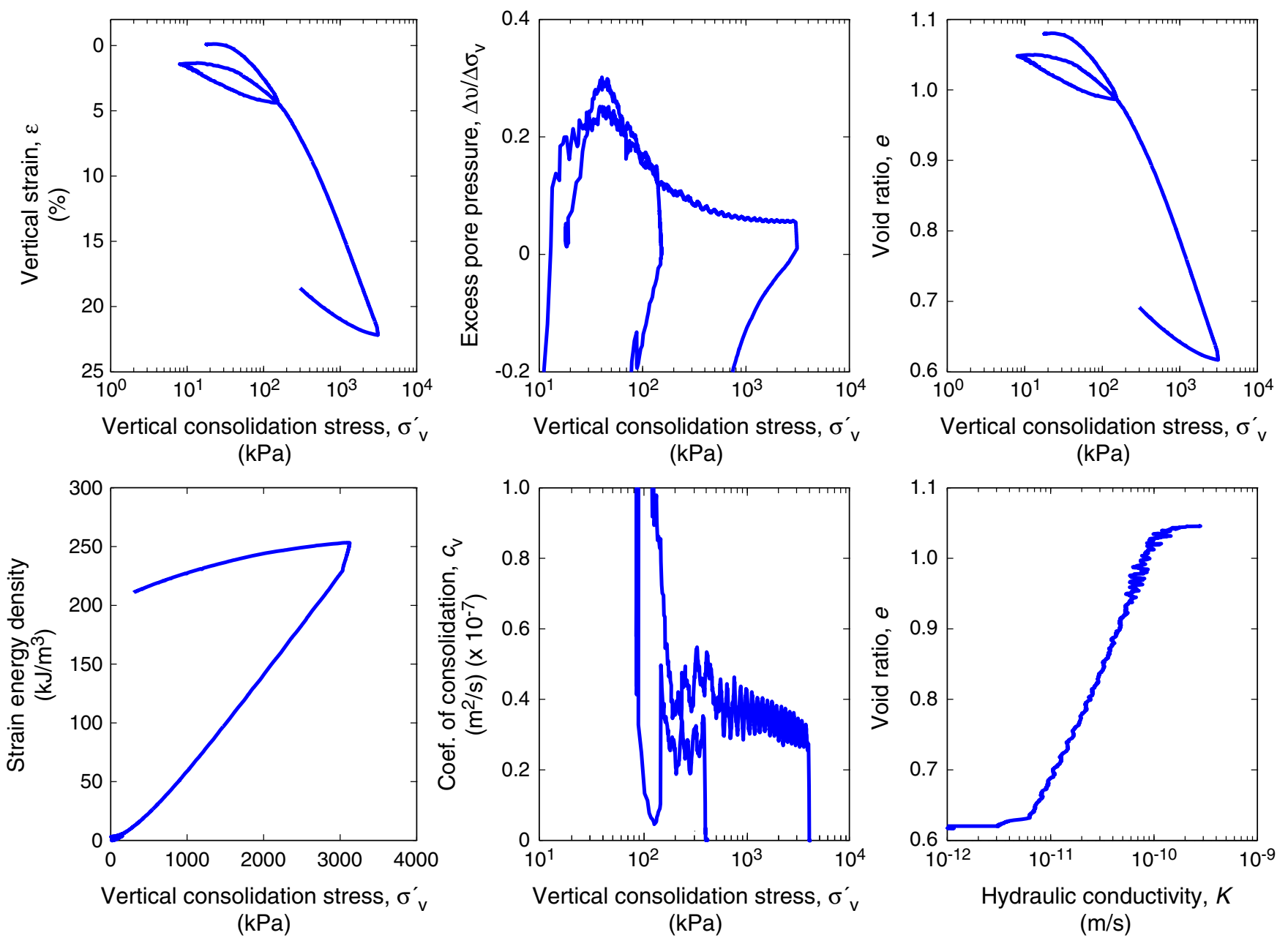

$(\mathrm{m} / \mathrm{s})$ 
Figure F17. CRS815 consolidation data for Sample 308-U1322B-4H-3WR, 27.21 mbsf. Coef. = coefficient.

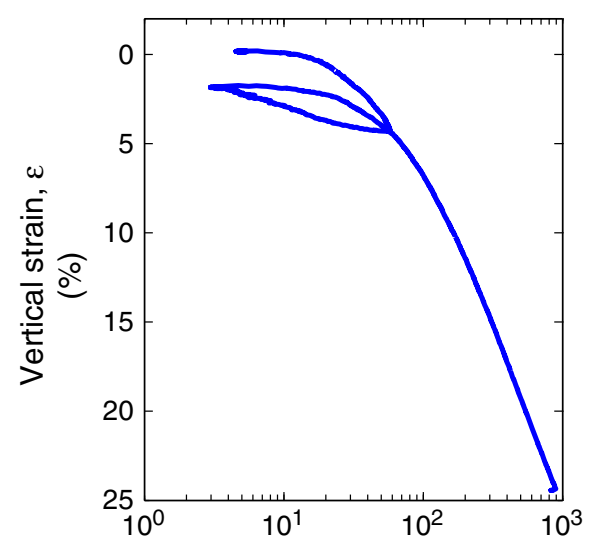

Vertical consolidation stress, $\sigma_{v}^{\prime}$ $(\mathrm{kPa})$

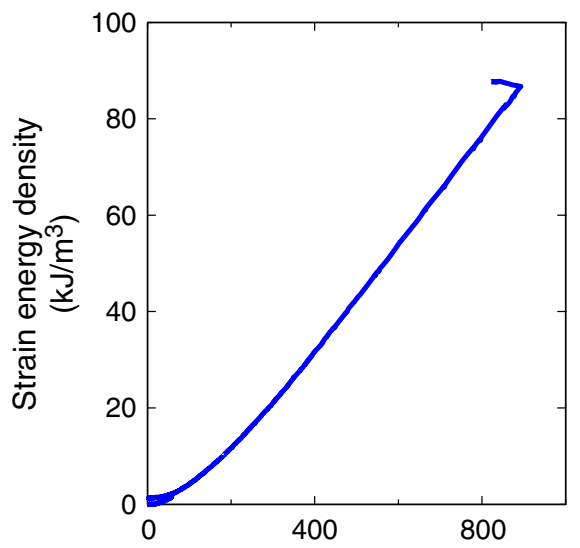

Vertical consolidation stress, $\sigma_{v}^{\prime}$ (kPa)

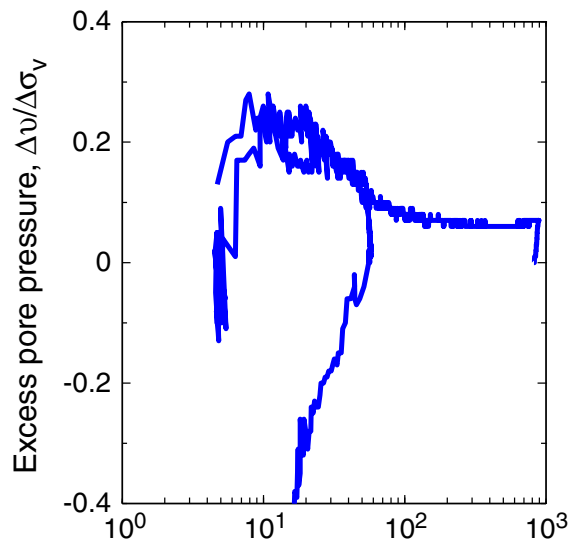

Vertical consolidation stress, $\sigma_{\text {v }}^{\prime}$ (kPa)

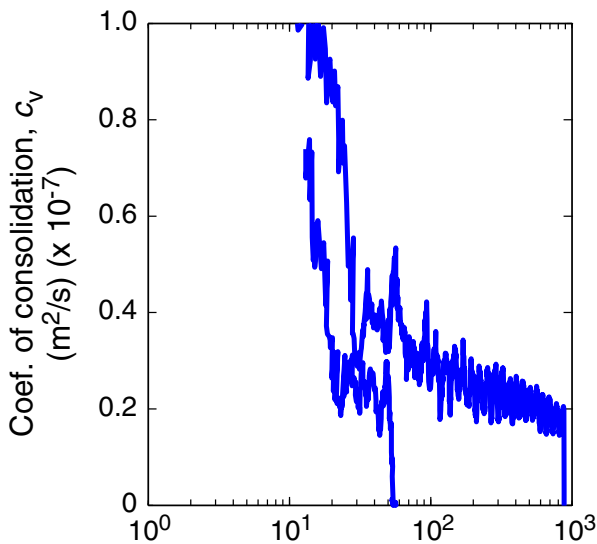

Vertical consolidation stress, $\sigma_{v}^{\prime}$ ( $\mathrm{kPa})$

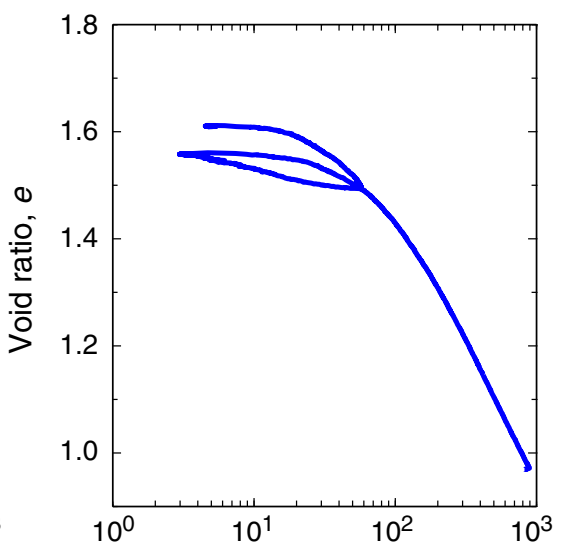

Vertical consolidation stress, $\sigma_{\mathrm{v}}^{\prime}$ (kPa)

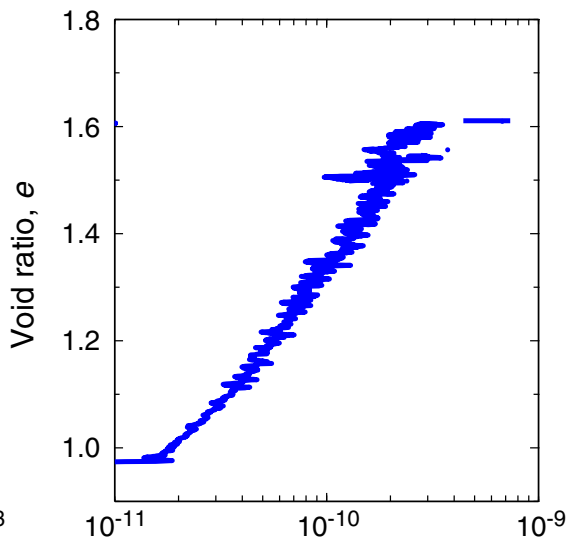

Hydraulic conductivity, $K$ $(\mathrm{m} / \mathrm{s})$ 
Figure F18. CRS824 consolidation data for Sample 308-U1322B-25H-6WR, 209.81 mbsf. Coef. = coefficient.

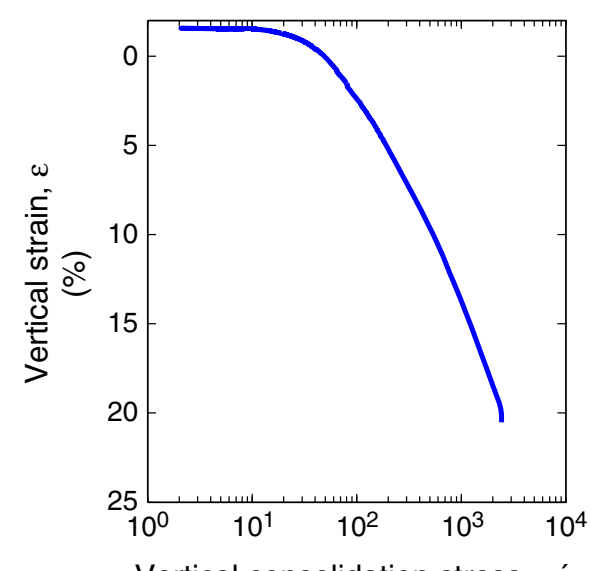

Vertical consolidation stress, $\sigma_{v}^{\prime}$ (kPa)

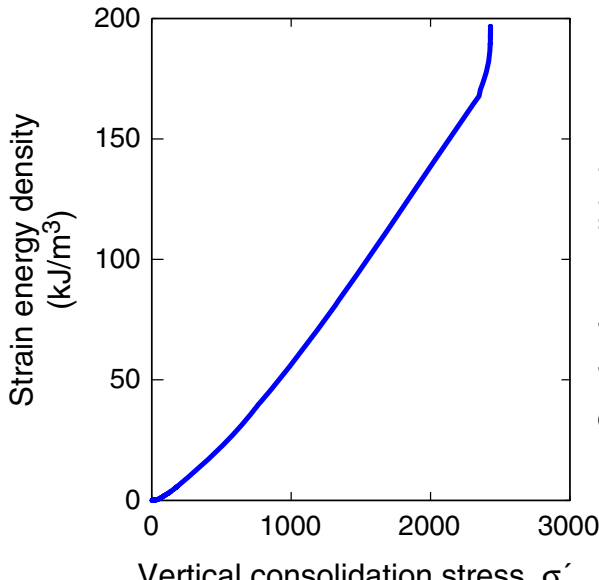

(kPa)

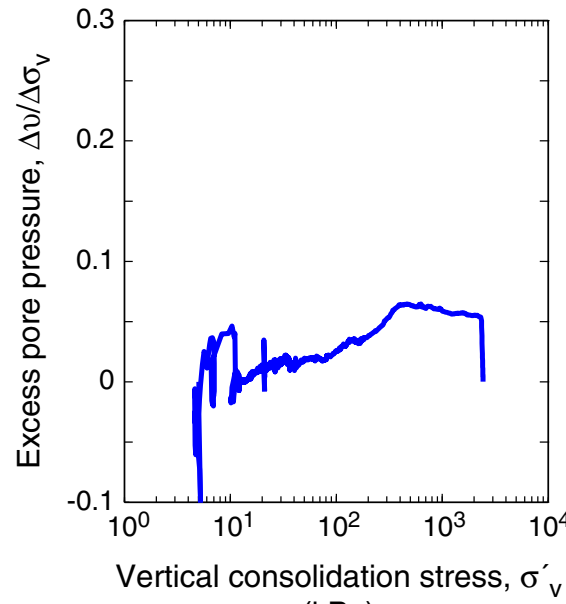

(kPa)

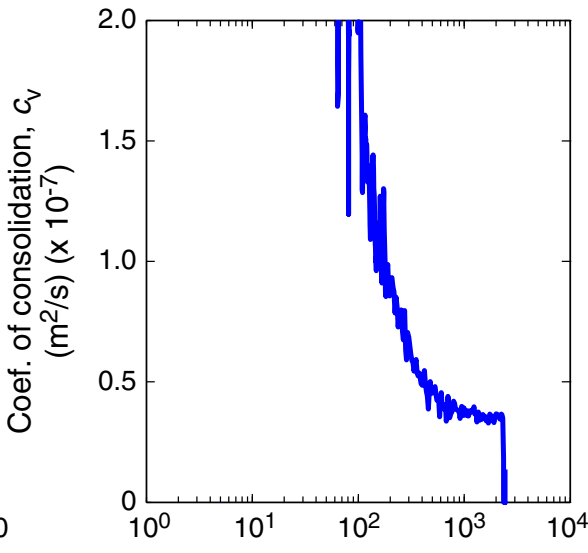

Vertical consolidation stress, $\sigma_{\text {v }}^{\prime}$ (kPa)

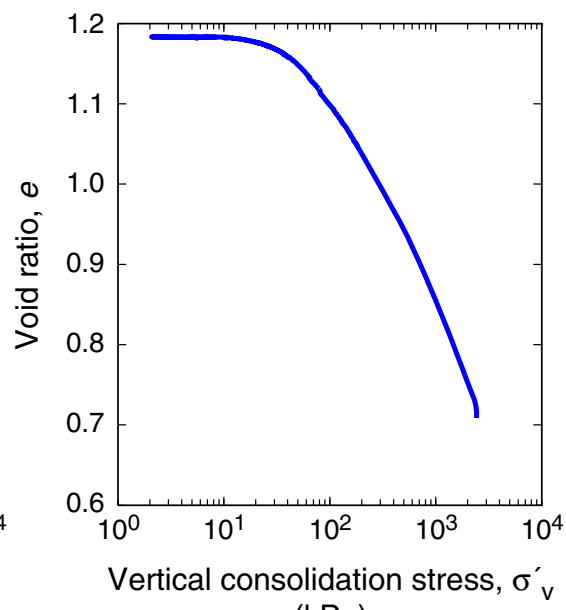

(kPa)

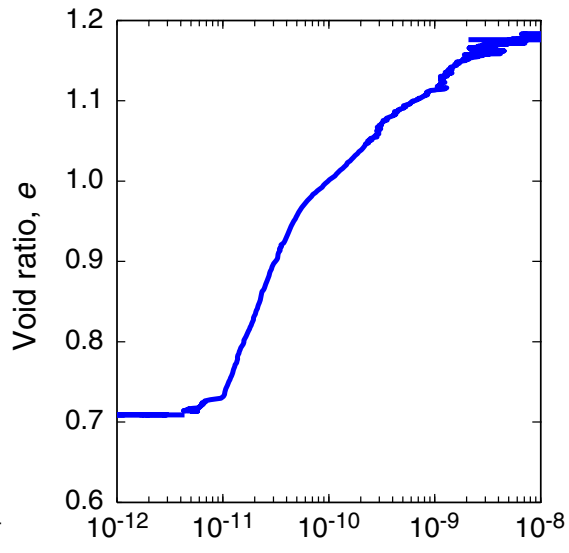

Hydraulic conductivity, $K$ $(\mathrm{m} / \mathrm{s})$ 
Figure F19. CRS825 consolidation data for Sample 308-U1322B-21H-3WR, 178.7 mbsf. Coef. = coefficient.

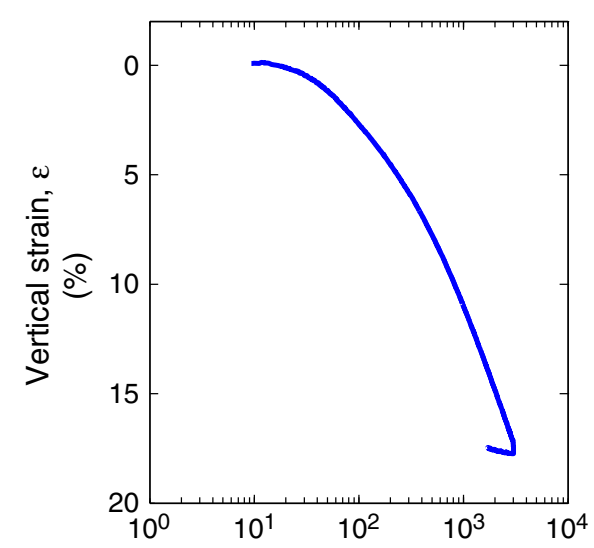

Vertical consolidation stress, $\sigma_{\mathrm{v}}^{\prime}$ $(\mathrm{kPa})$

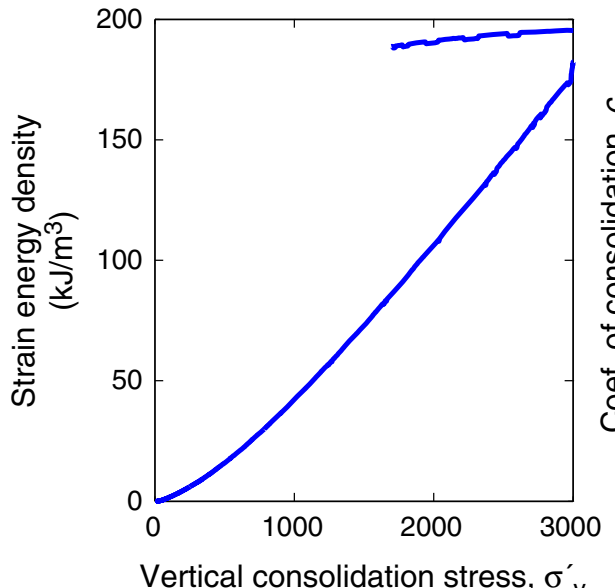

$(\mathrm{kPa})$

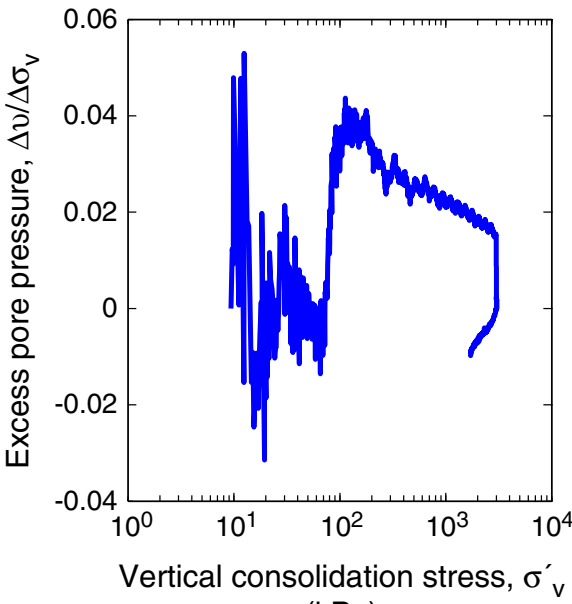

(kPa)

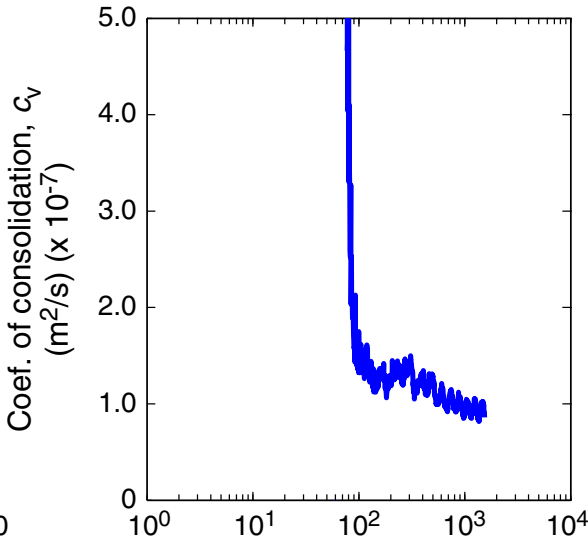

Vertical consolidation stress, $\sigma^{\prime}$ v (kPa)

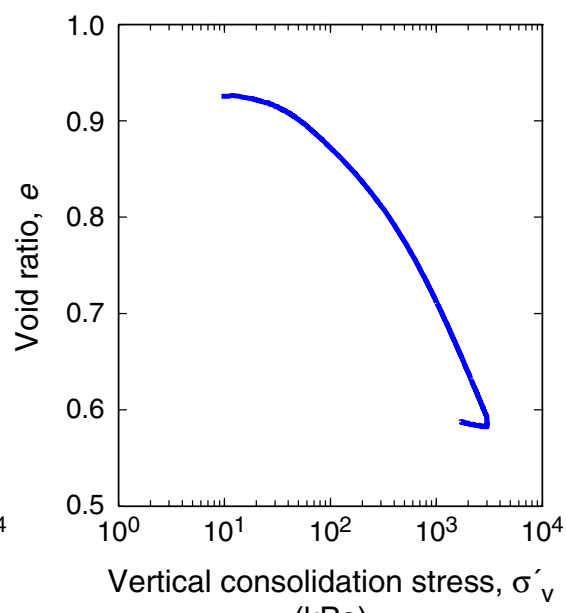

(kPa)

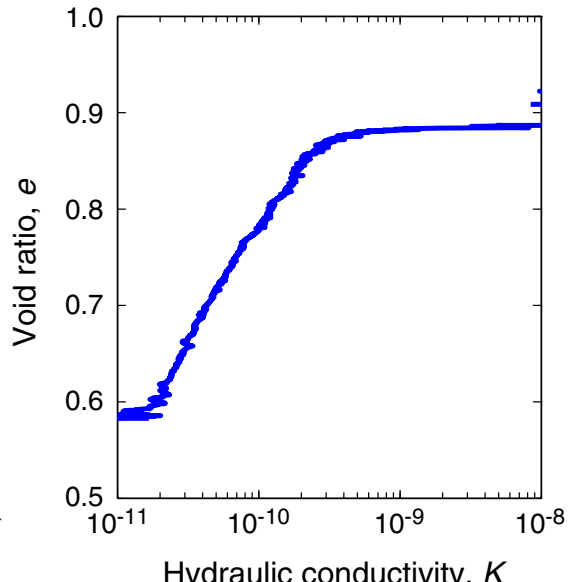

$(\mathrm{m} / \mathrm{s})$ 
Figure F20. CRS826 consolidation data for Sample 308-U1322D-1H-2WR, 42.87 mbsf. Coef. = coefficient.

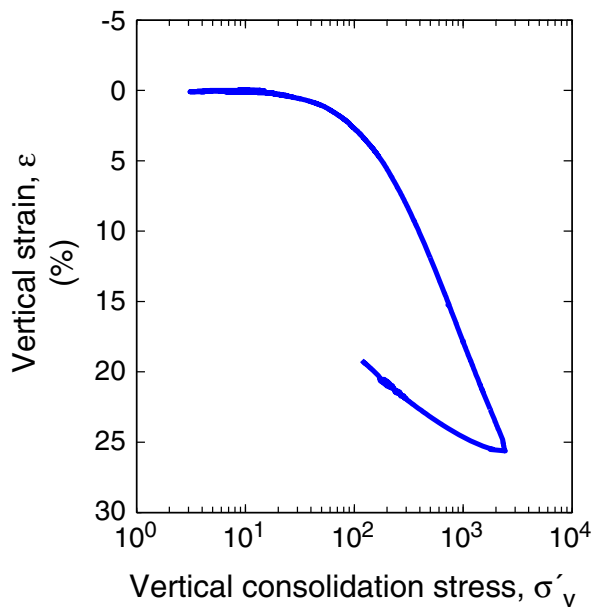

$(\mathrm{kPa})$

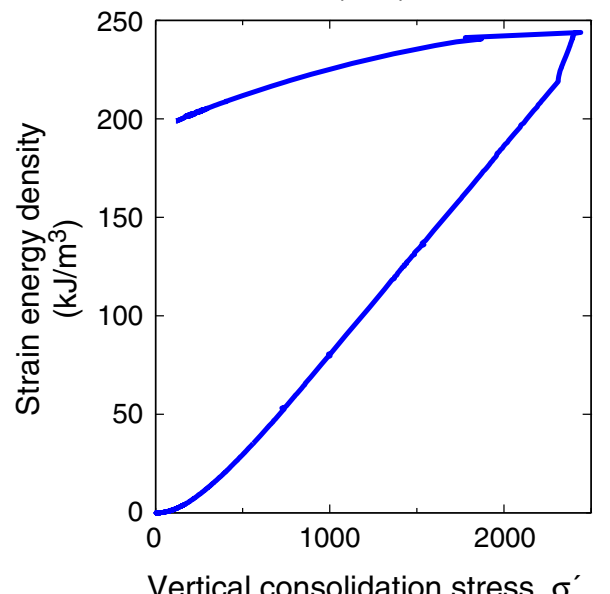

(kPa)

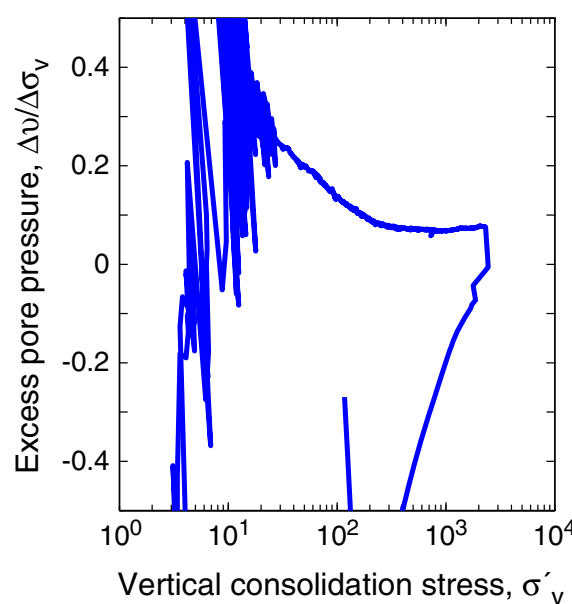

(kPa)

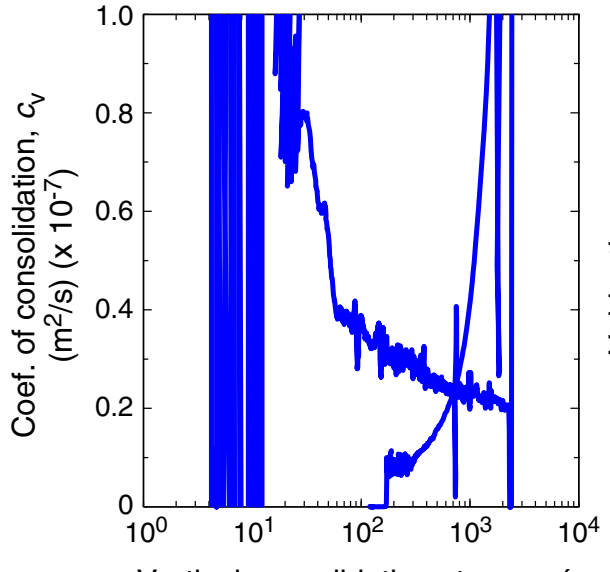

Vertical consolidation stress, $\sigma^{\prime}$ v (kPa)

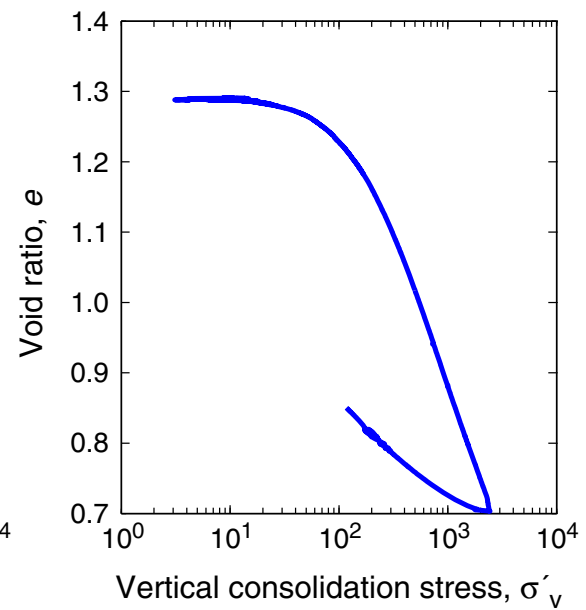

$(\mathrm{kPa})$

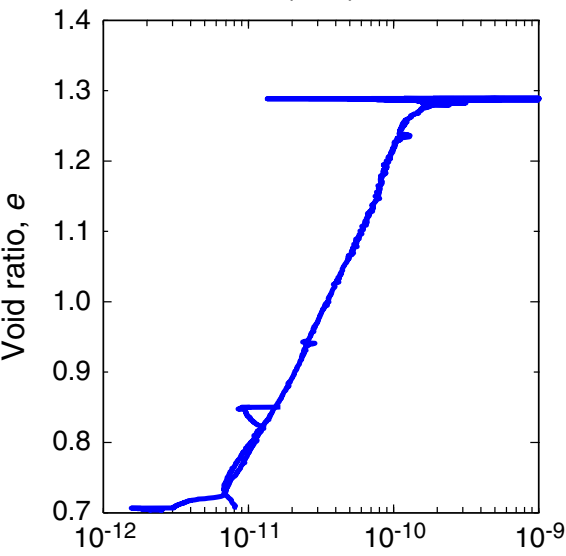

Hydraulic conductivity, $K$ $(\mathrm{m} / \mathrm{s})$ 
Figure F21. CRS001 consolidation data for Sample 308-U1324C-6H-3WR, 304.02 mbsf. Coef. = coefficient.

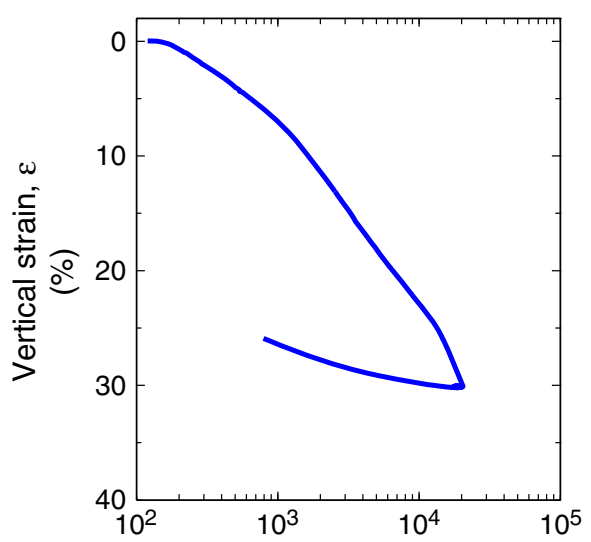

Vertical consolidation stress, $\sigma^{\prime}$ v (kPa)

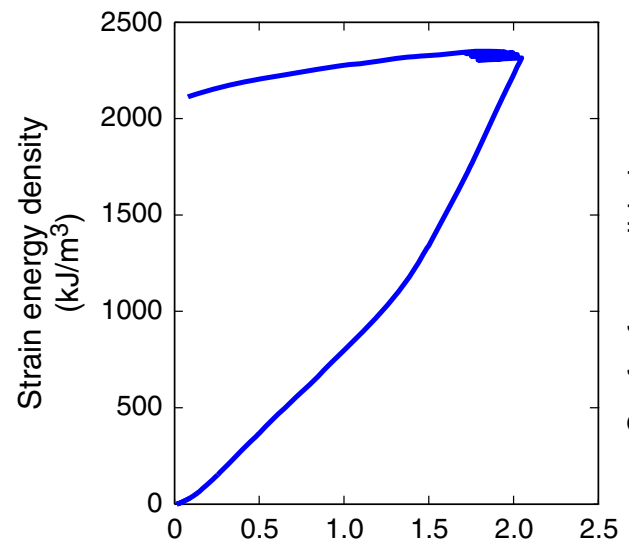

Vertical consolidation stress, $\sigma^{\prime}$ v $(\mathrm{kPa})\left(\times 10^{4}\right)$

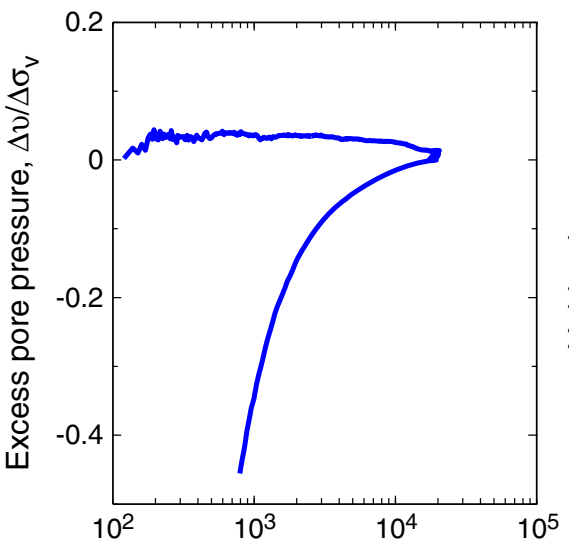

Vertical consolidation stress, $\sigma_{\text {v }}^{\prime}$ $(\mathrm{kPa})$

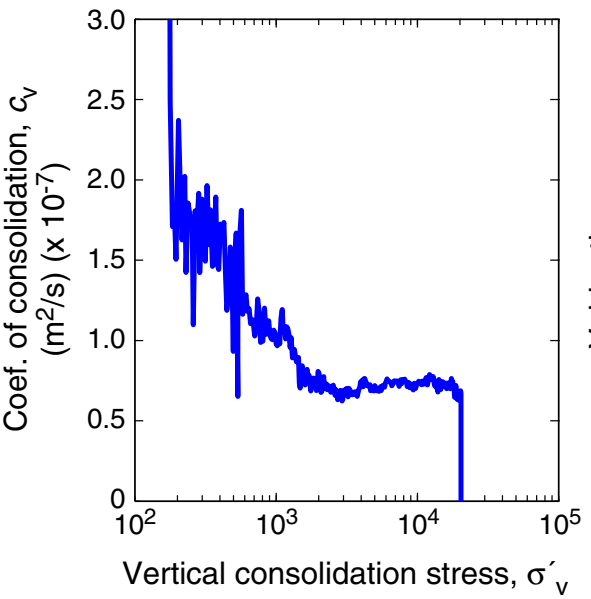

$(\mathrm{kPa})$

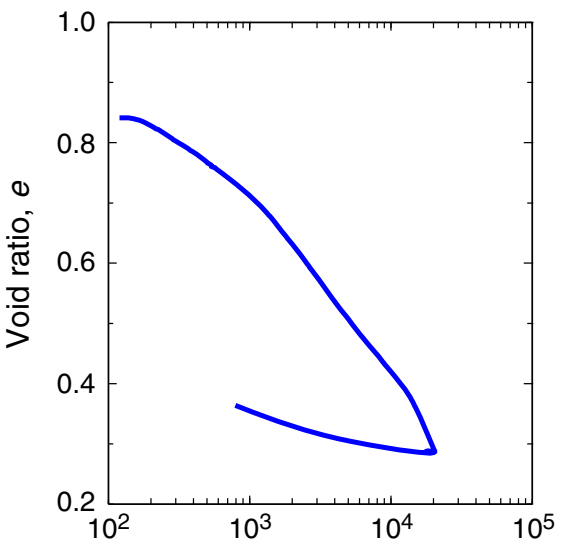

Vertical consolidation stress, $\sigma_{v}^{\prime}$ (kPa)

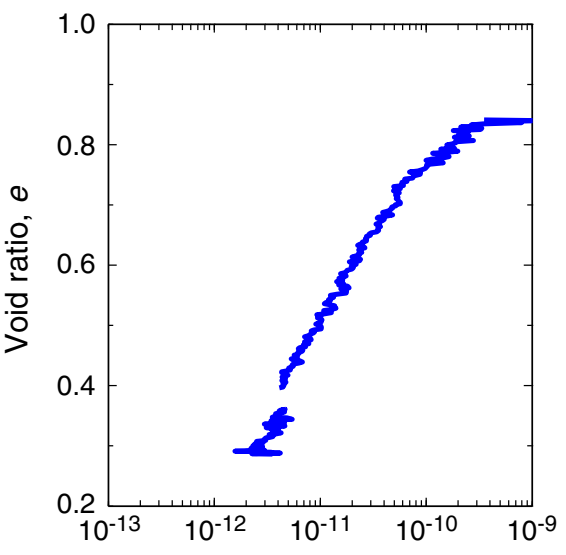

Hydraulic conductivity, $K$ $(\mathrm{m} / \mathrm{s})$ 
Figure F22. CRS002 consolidation data for Sample 308-U1324C-6H-3WR, 303.94 mbsf. Coef. = coefficient.

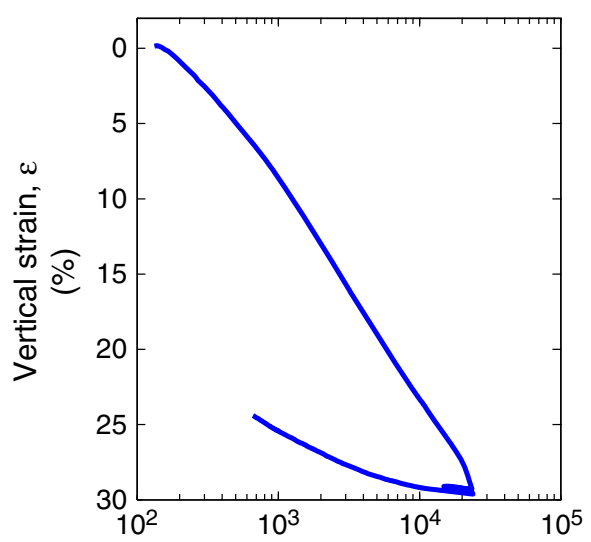

Vertical consolidation stress, $\sigma_{\mathrm{v}}^{\prime}$ (kPa)

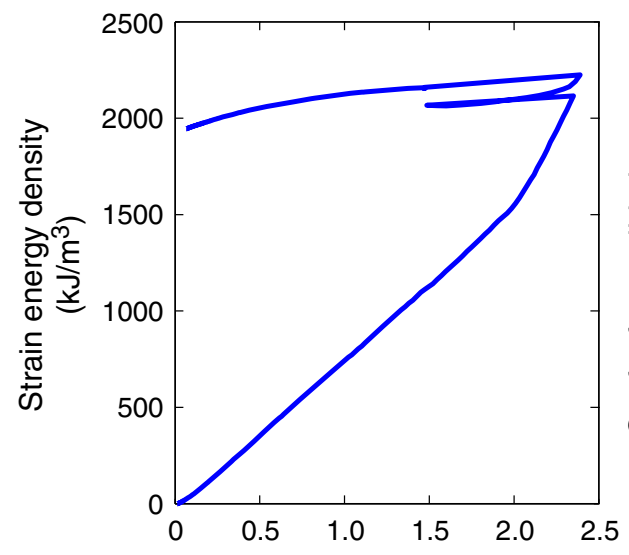

Vertical consolidation stress, $\sigma^{\prime}$ v $(\mathrm{kPa})\left(\times 10^{4}\right)$

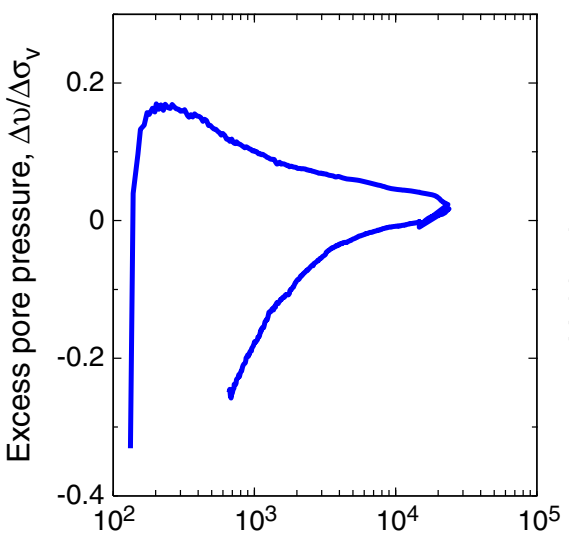

Vertical consolidation stress, $\sigma_{\text {v }}^{\prime}$ (kPa)

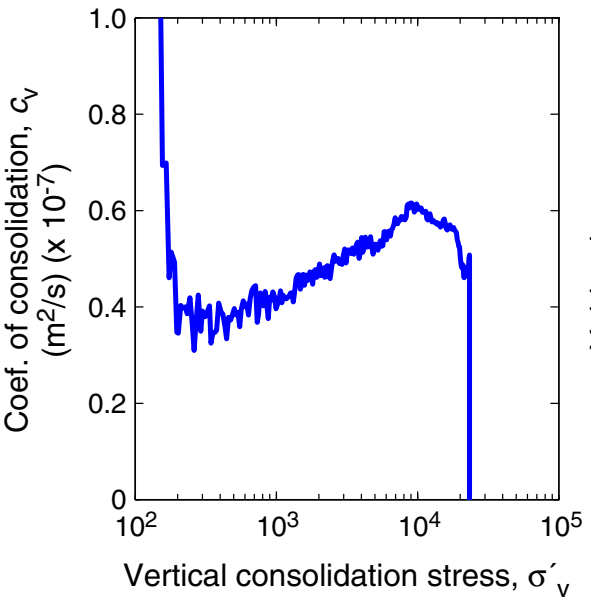

(kPa)

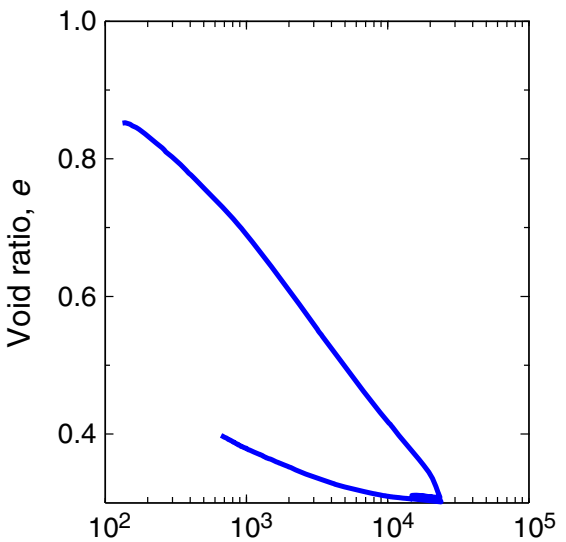

Vertical consolidation stress, $\sigma_{v}^{\prime}$ (kPa)

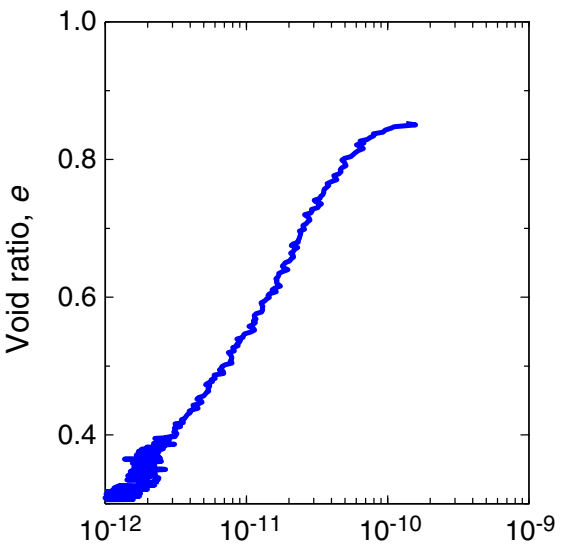

Hydraulic conductivity, $K$ $(\mathrm{m} / \mathrm{s})$ 
Figure F23. CRS003 consolidation data for Sample 308-U1324C-1H-1WR, 51.21 mbsf. Coef. = coefficient.

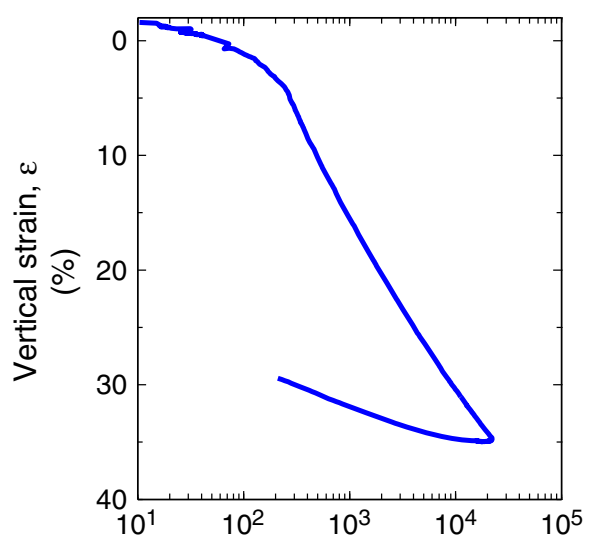

Vertical consolidation stress, $\sigma_{\mathrm{V}}^{\prime}$ (kPa)

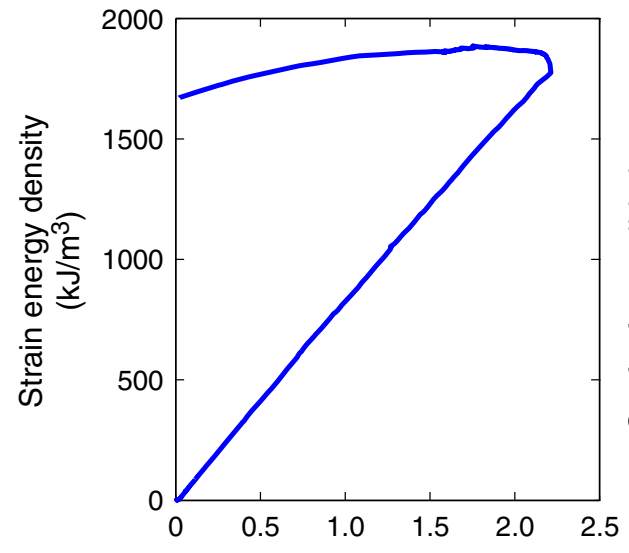

Vertical consolidation stress, $\sigma^{\prime}$ v $(\mathrm{kPa})\left(\times 10^{4}\right)$

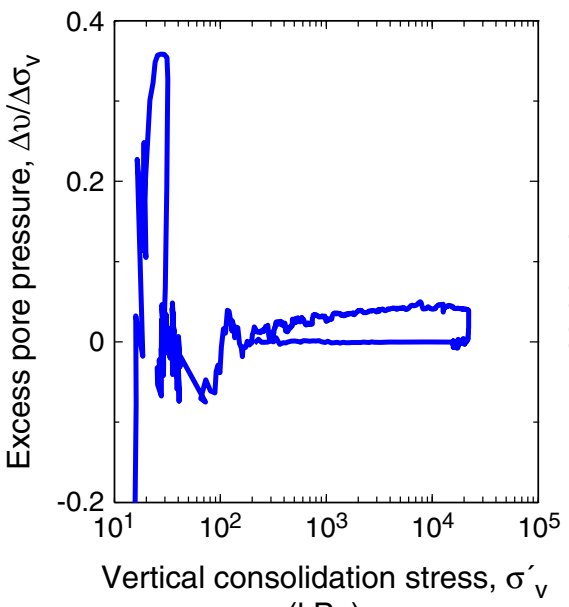

$(\mathrm{kPa})$

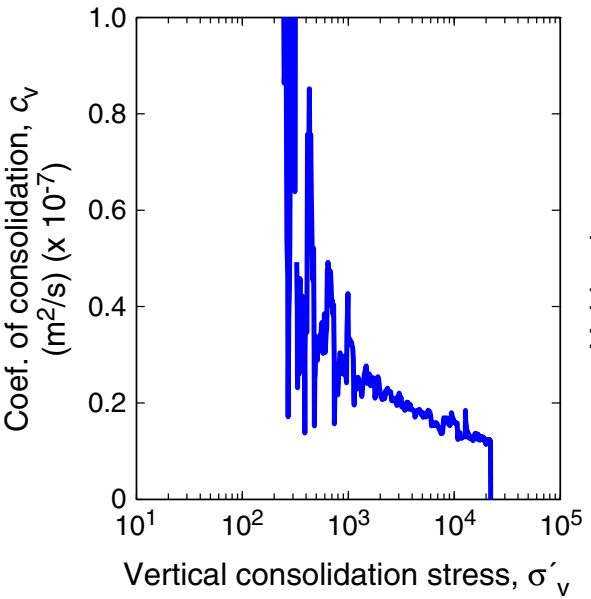

(kPa)

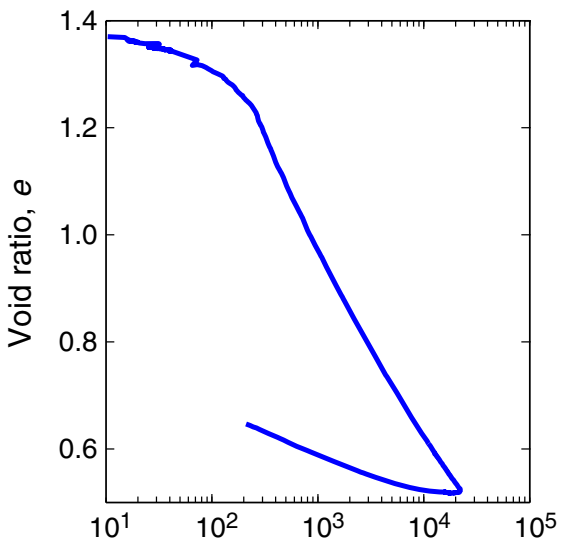

Vertical consolidation stress, $\sigma_{v}^{\prime}$ $(\mathrm{kPa})$

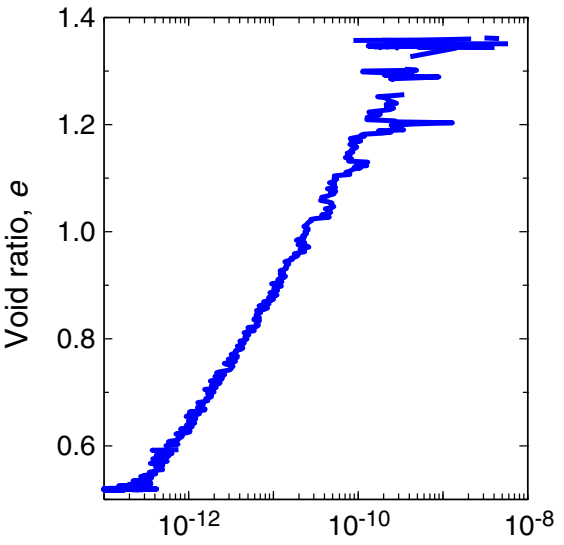

Hydraulic conductivity, $K$ $(\mathrm{m} / \mathrm{s})$ 
Figure F24. CRS004 consolidation data for Sample 308-U1324C-1H-1WR, 51.14 mbsf. Coef. = coefficient.

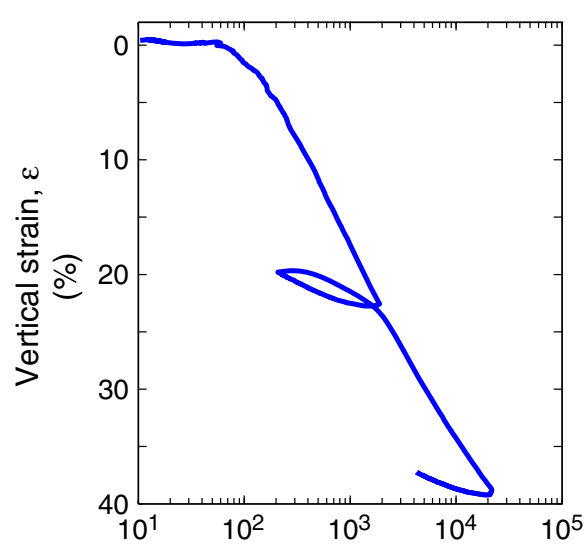

Vertical consolidation stress, $\sigma^{\prime}$ v $(\mathrm{kPa})$

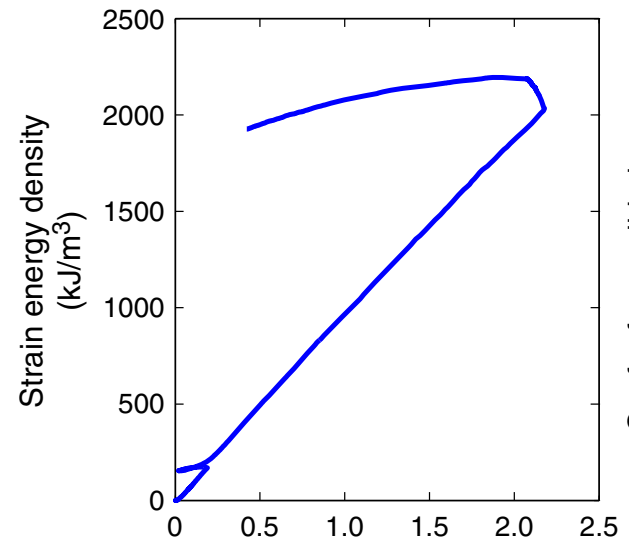

Vertical consolidation stress, $\sigma_{\mathrm{v}}^{\prime}$ $(\mathrm{kPa})\left(\mathrm{x} \mathrm{10} 0^{4}\right)$

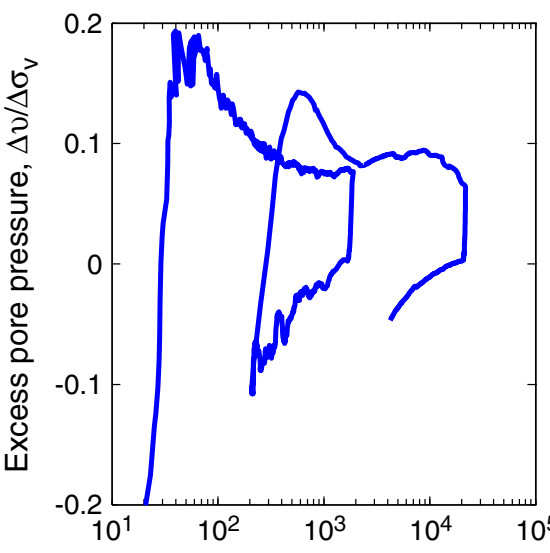

Vertical consolidation stress, $\sigma_{\mathrm{v}}^{\prime}$ $(\mathrm{kPa})$

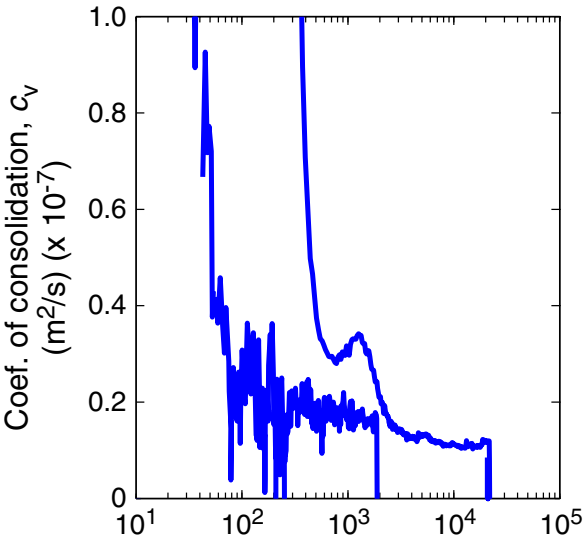

Vertical consolidation stress, $\sigma^{\prime}$ v $(\mathrm{kPa})$

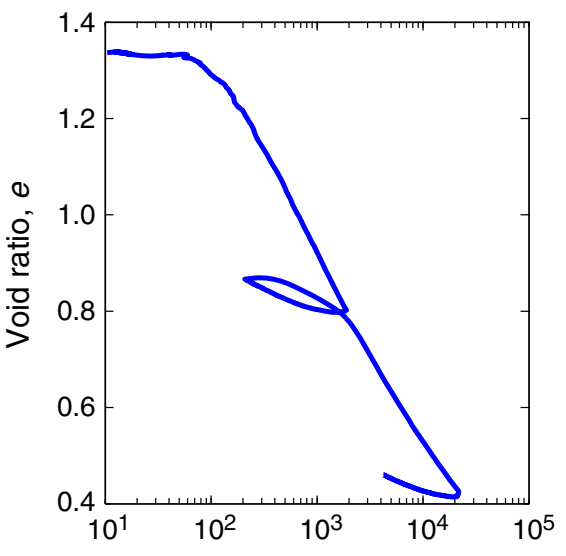

Vertical consolidation stress, $\sigma_{\text {v }}^{\prime}$ (kPa)

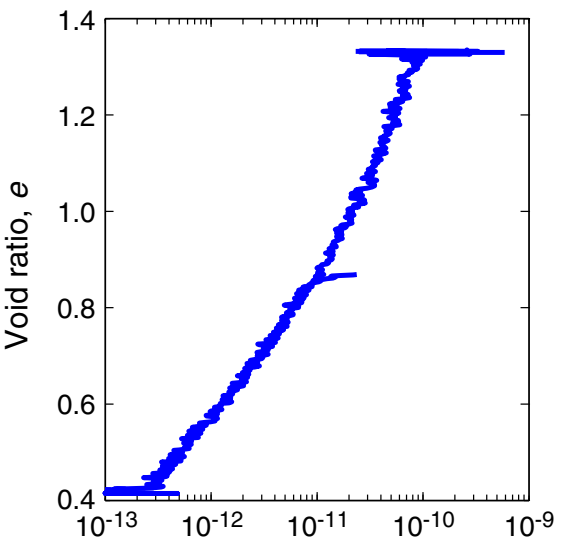

Hydraulic conductivity, $K$ $(\mathrm{m} / \mathrm{s})$ 
Figure F25. CRS005 consolidation data for Sample 308-U1324B-13H-7WR, 117.4 mbsf. Coef. = coefficient.

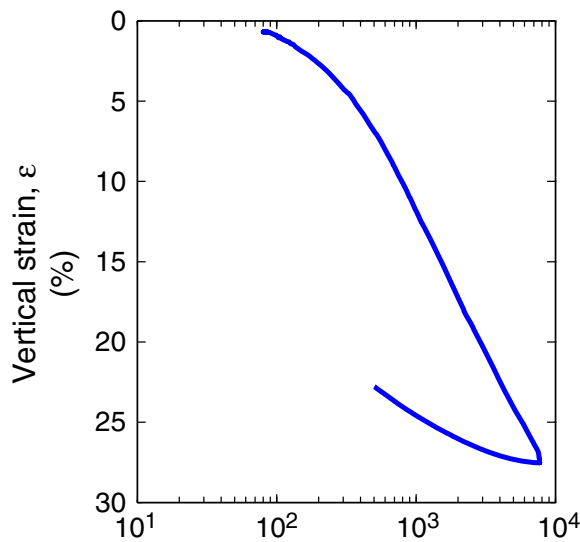

Vertical consolidation stress, $\sigma_{\mathrm{v}}^{\prime}$ ( $\mathrm{kPa})$

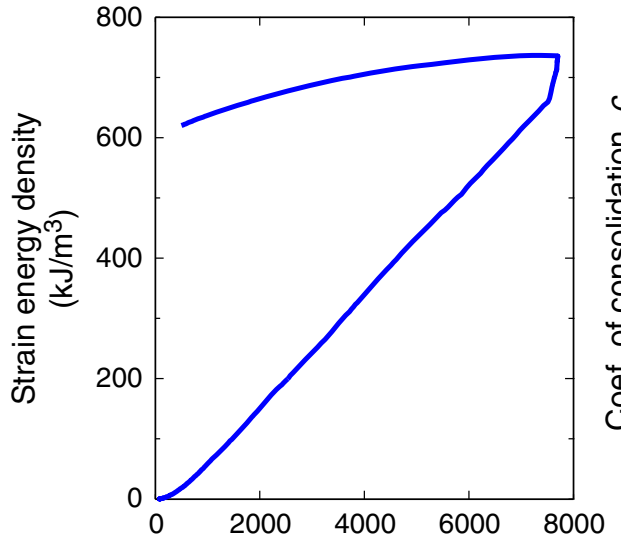

Vertical consolidation stress, $\sigma^{\prime}$ v

(kPa)

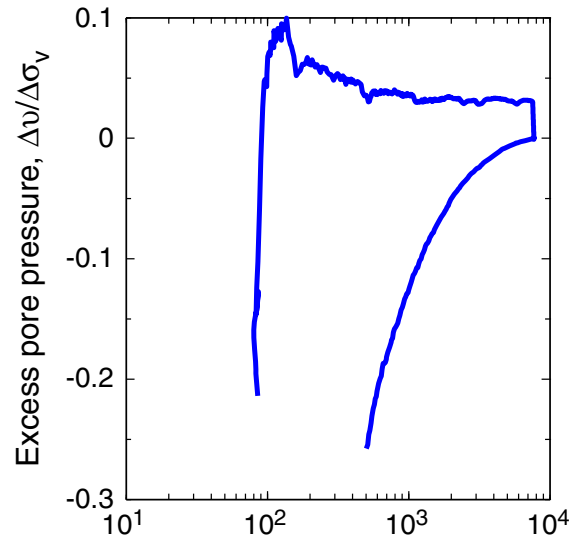

Vertical consolidation stress, $\sigma_{\mathrm{v}}^{\prime}$ (kPa)

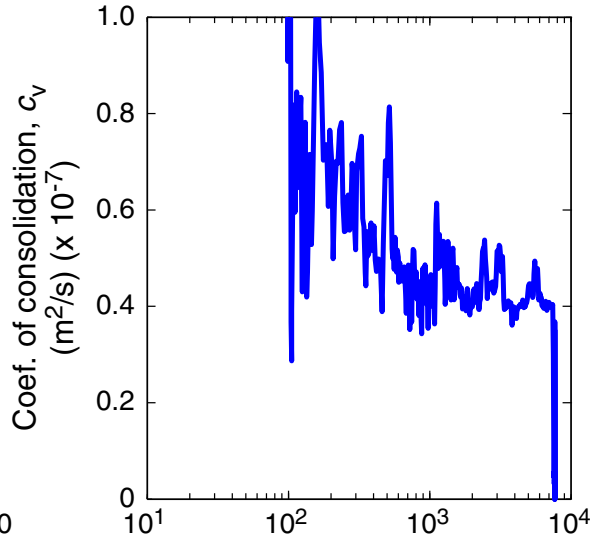

Vertical consolidation stress, $\sigma_{\text {v }}^{\prime}$ (kPa)

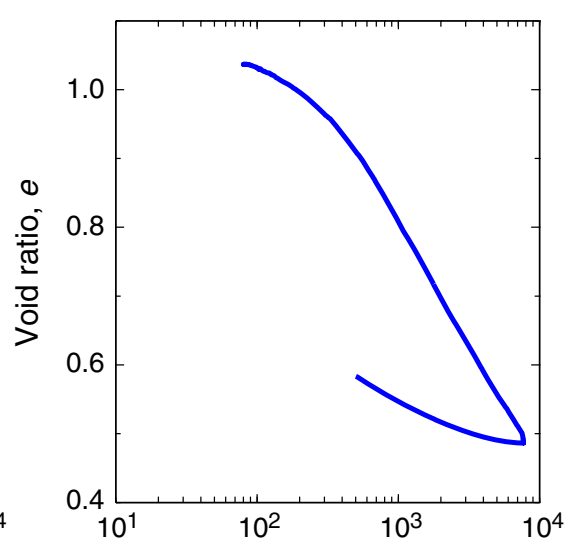

Vertical consolidation stress, $\sigma_{v}^{\prime}$ (kPa)

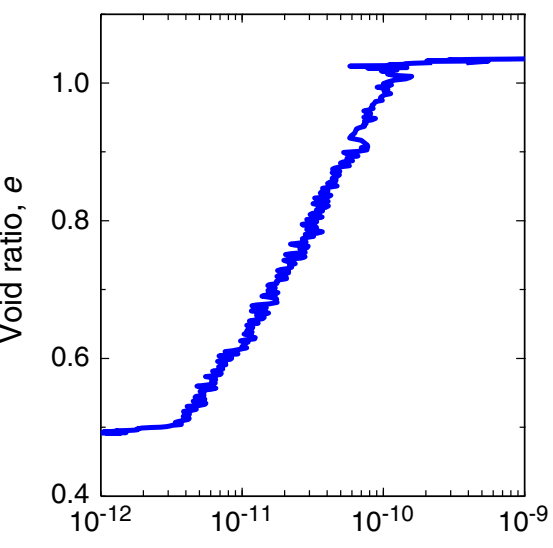

Hydraulic conductivity, $K$ $(\mathrm{m} / \mathrm{s})$ 
Figure F26. CRS006 consolidation data for Sample 308-U1324B-70X-6WR, 578.13 mbsf. Coef. = coefficient.

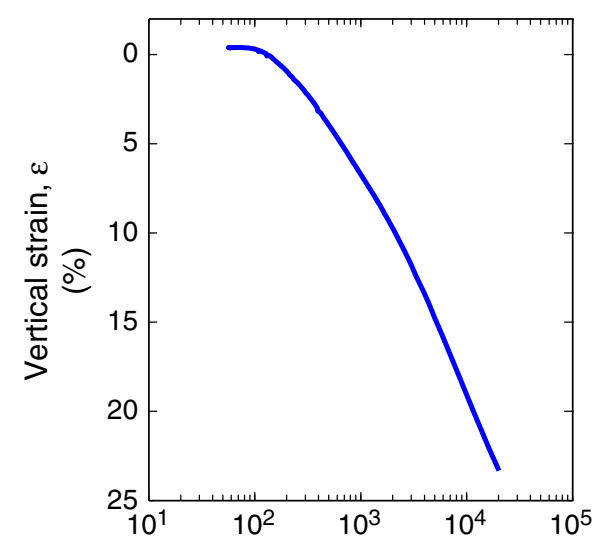

Vertical consolidation stress, $\sigma_{v}^{\prime}$ (kPa)

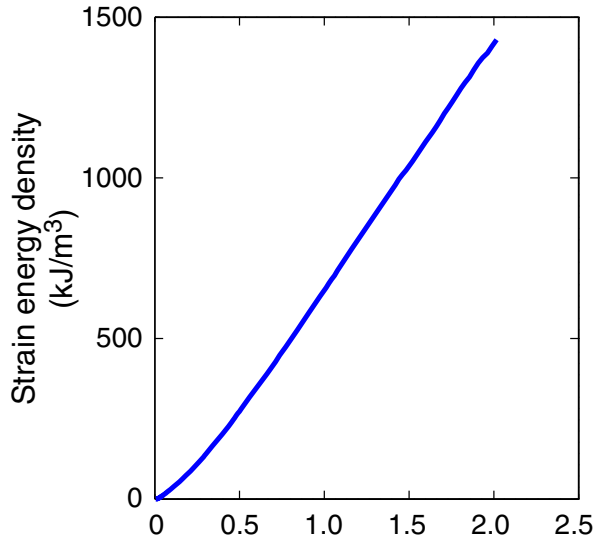

Vertical consolidation stress, $\sigma^{\prime}$ v $(\mathrm{kPa})\left(\mathrm{x} \mathrm{10} 0^{4}\right)$

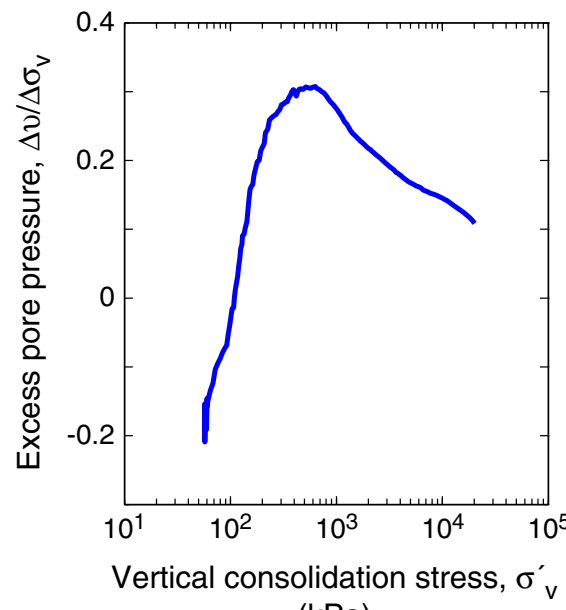

(kPa)

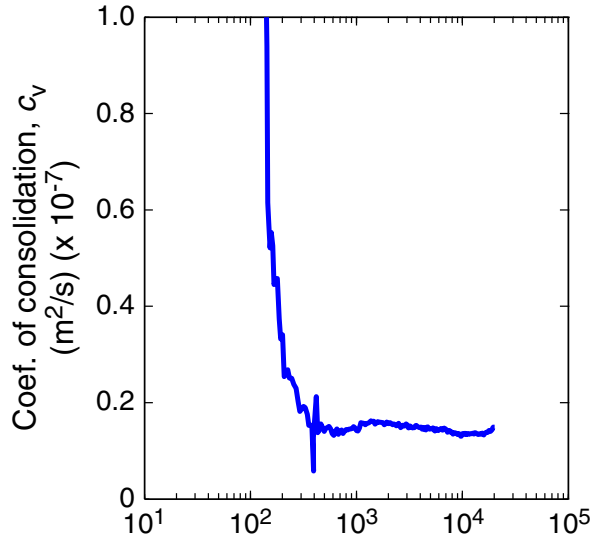

Vertical consolidation stress, $\sigma_{\text {v }}^{\prime}$ (kPa)

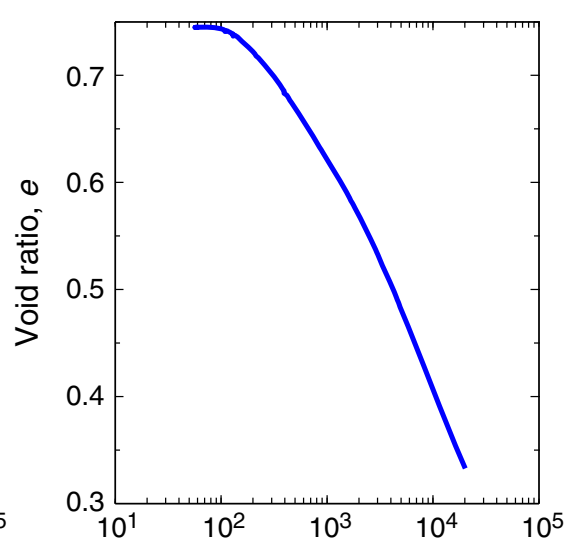

Vertical consolidation stress, $\sigma_{v}^{\prime}$ (kPa)

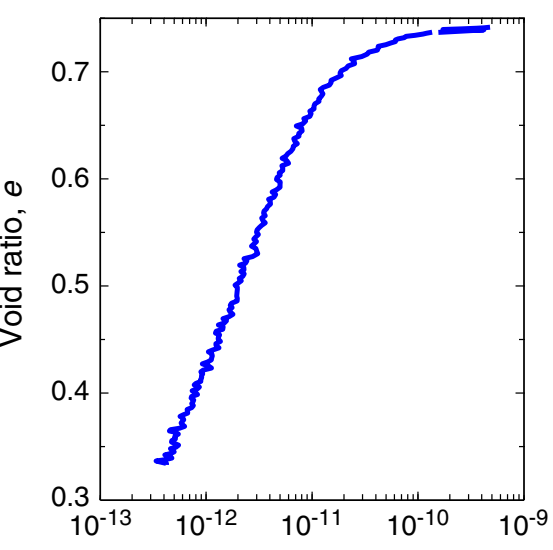

Hydraulic conductivity, $K$ $(\mathrm{m} / \mathrm{s})$ 
Figure F27. CRS007 consolidation data for Sample 308-U1324B-60X-2WR, 476.86 mbsf. Coef. = coefficient

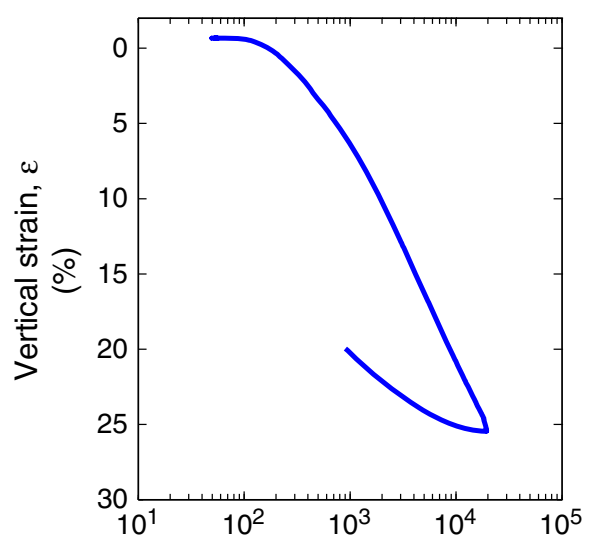

Vertical consolidation stress, $\sigma_{\mathrm{v}}^{\prime}$ $(\mathrm{kPa})$

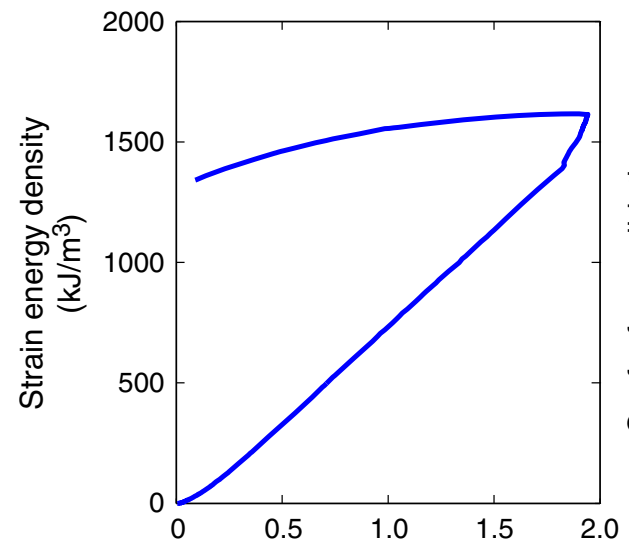

Vertical consolidation stress, $\sigma_{v}^{\prime}$ $(\mathrm{kPa})\left(\mathrm{x} \mathrm{10^{4 }}\right)$

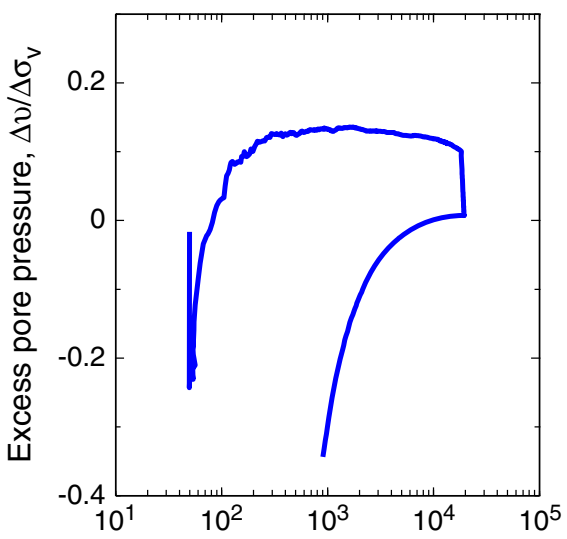

Vertical consolidation stress, $\sigma_{\mathrm{v}}^{\prime}$ $(\mathrm{kPa})$

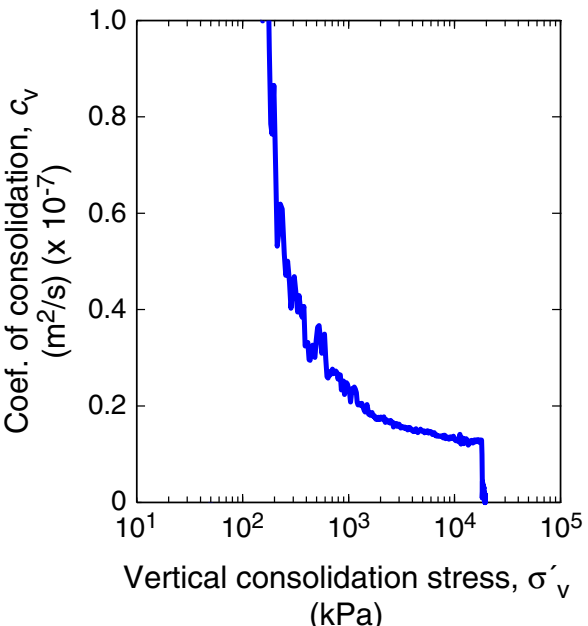

(kPa)

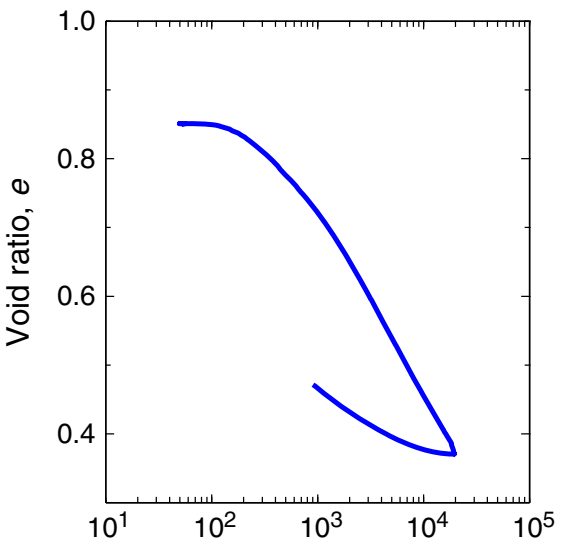

Vertical consolidation stress, $\sigma_{v}^{\prime}$ $(\mathrm{kPa})$

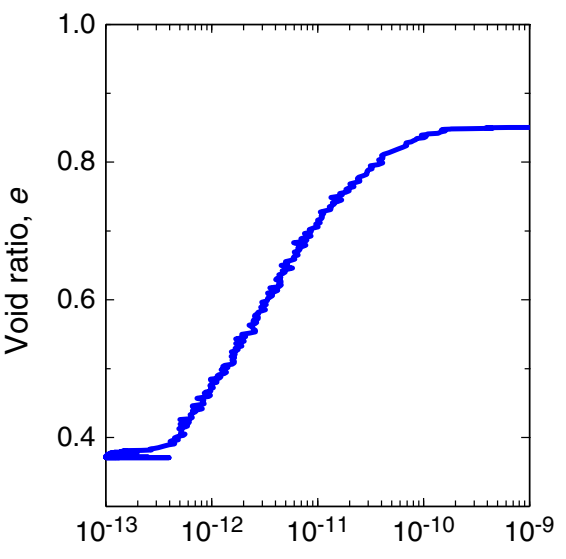

Hydraulic conductivity, $K$ $(\mathrm{m} / \mathrm{s})$ 
Figure F28. CRS008 consolidation data for Sample 308-U1324C-7H-1WR, 405.81 mbsf. Coef. = coefficient.

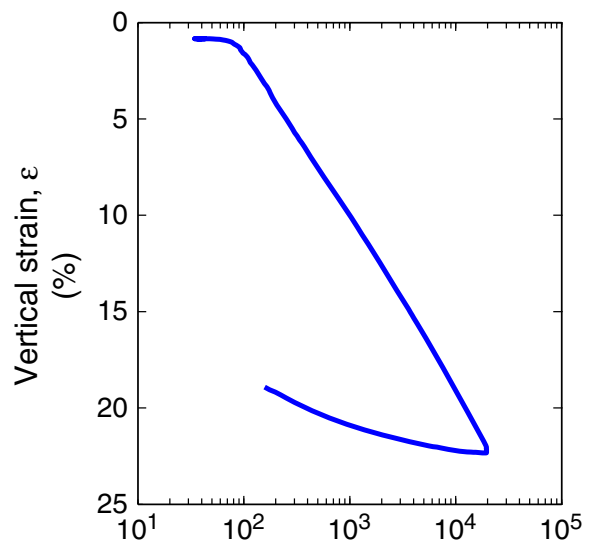

Vertical consolidation stress, $\sigma_{\mathrm{v}}^{\prime}$ (kPa)

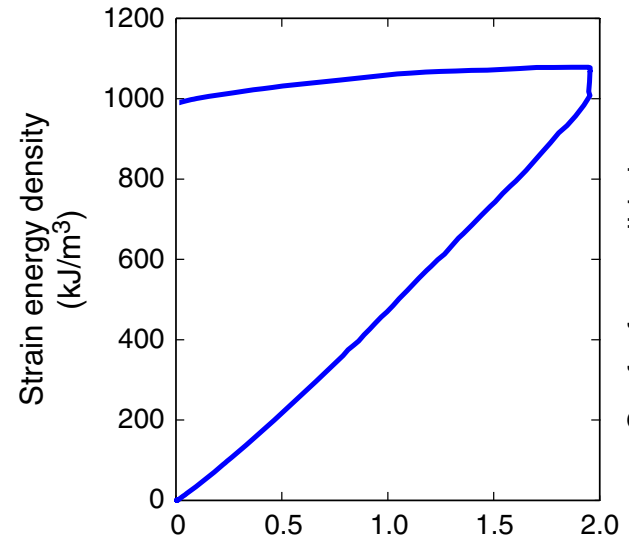

Vertical consolidation stress, $\sigma^{\prime}$ v $(\mathrm{kPa})\left(\mathrm{x} \mathrm{10^{4 }}\right)$

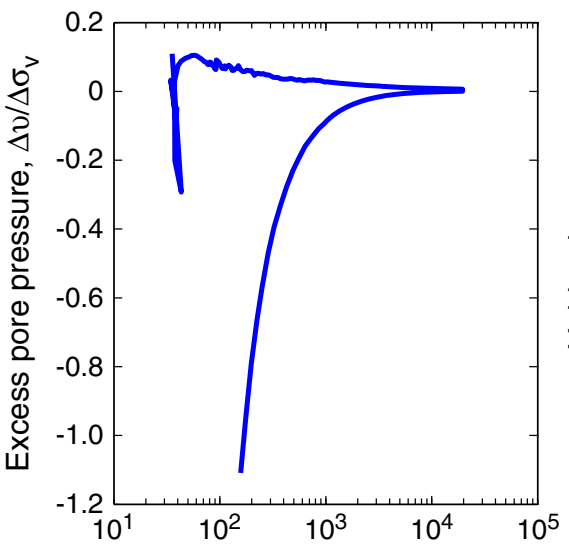

Vertical consolidation stress, $\sigma^{\prime}$ $(\mathrm{kPa})$

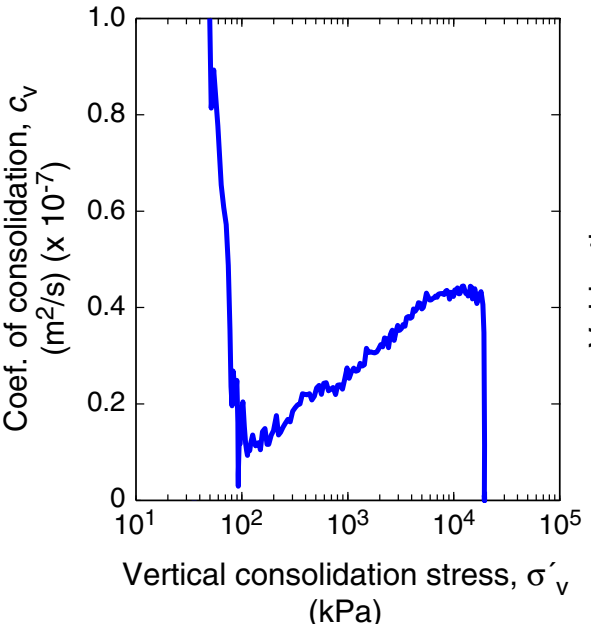

$(\mathrm{kPa})$

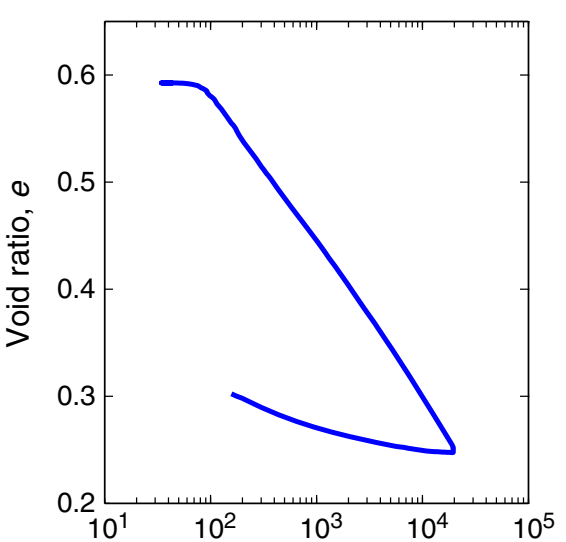

Vertical consolidation stress, $\sigma_{v}^{\prime}$ (kPa)

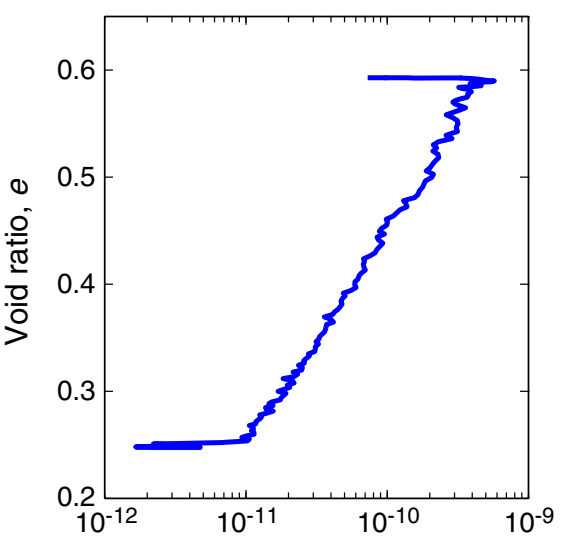

Hydraulic conductivity, $K$ $(\mathrm{m} / \mathrm{s})$ 
Figure F29. CRS013 consolidation data for Sample 308-U1324B-4H-7WR, 32.14 mbsf. Coef. = coefficient.

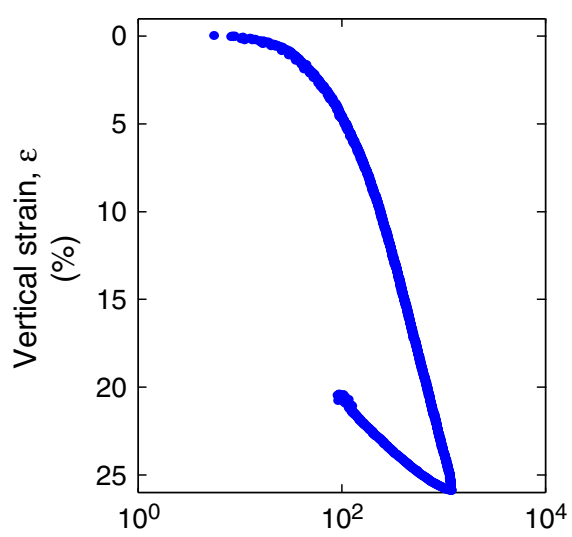

Vertical consolidation stress, $\sigma_{\mathrm{v}}^{\prime}$ $(\mathrm{kPa})$

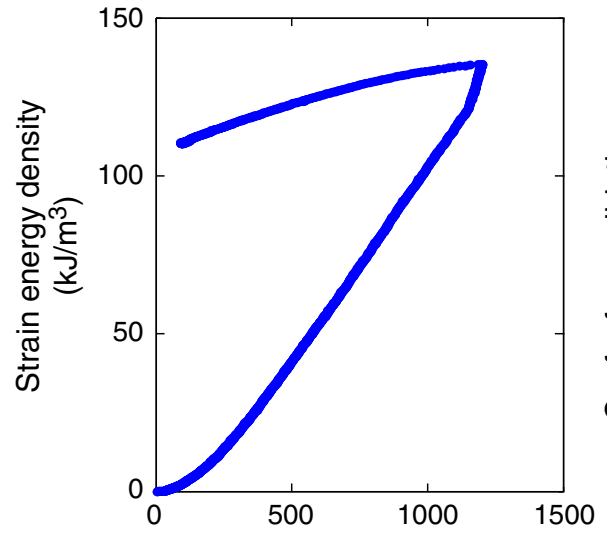

Vertical consolidation stress, $\sigma^{\prime}$ v (kPa)

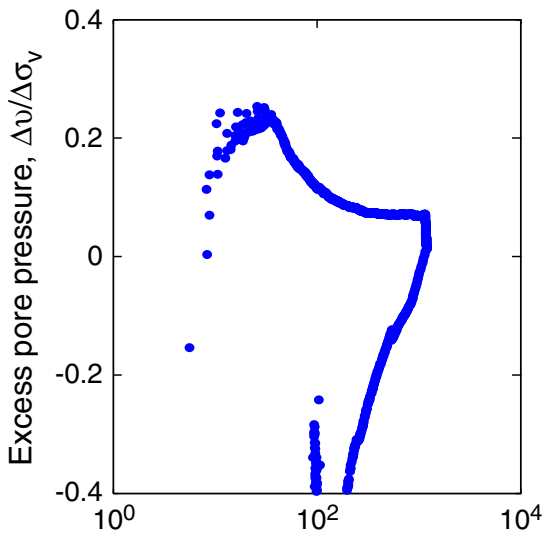

Vertical consolidation stress, $\sigma_{\mathrm{v}}^{\prime}$ (kPa)

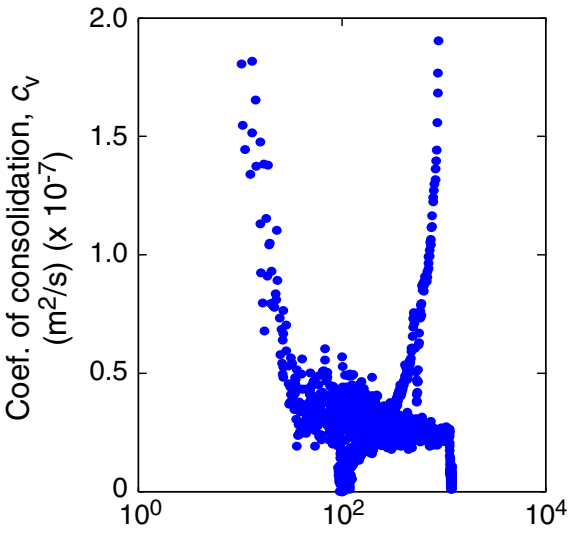

Vertical consolidation stress, $\sigma_{\mathrm{v}}^{\prime}$ (kPa)

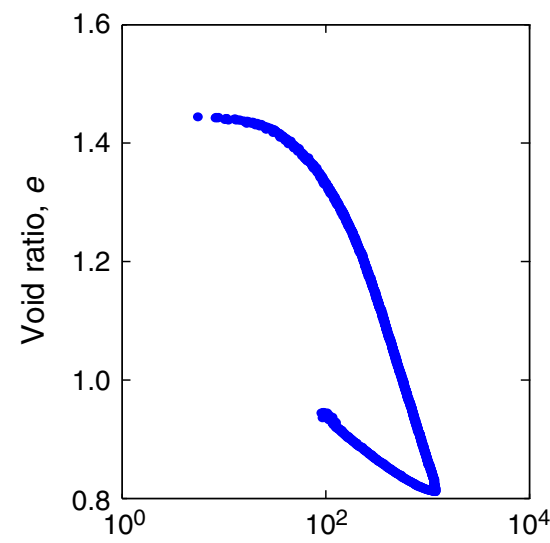

Vertical consolidation stress, $\sigma_{\mathrm{v}}^{\prime}$ ( $\mathrm{kPa})$

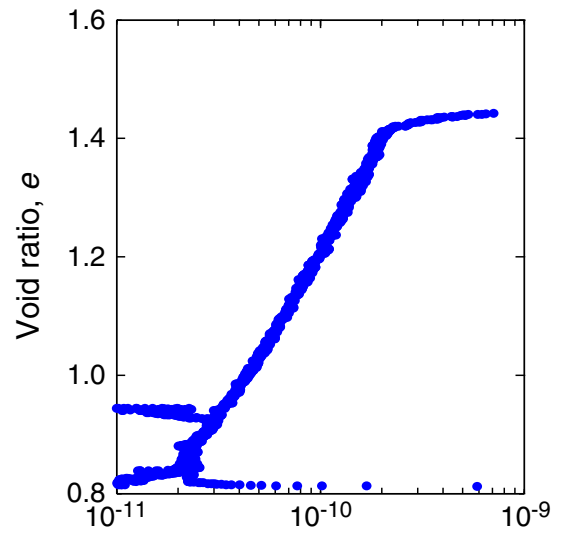

Hydraulic conductivity, $K$ $(\mathrm{m} / \mathrm{s})$ 
Figure F30. CRS014 consolidation data for Sample 308-U1324B-4H-7WR, 32.10 mbsf. Coef. = coefficient.

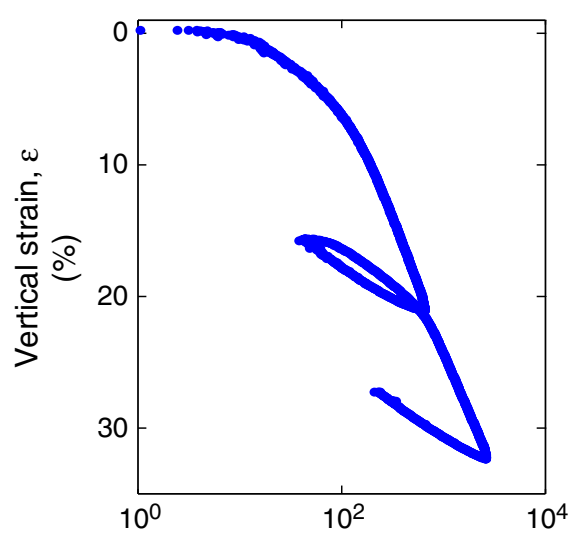

Vertical consolidation stress, $\sigma_{v}^{\prime}$ $(\mathrm{kPa})$

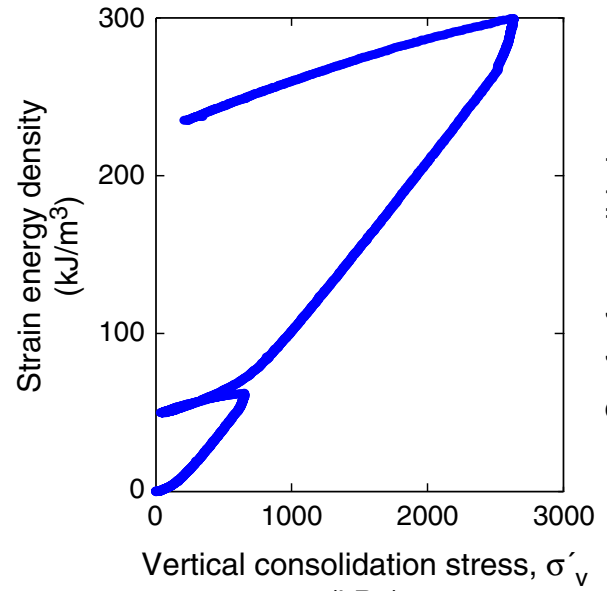

$(\mathrm{kPa})$

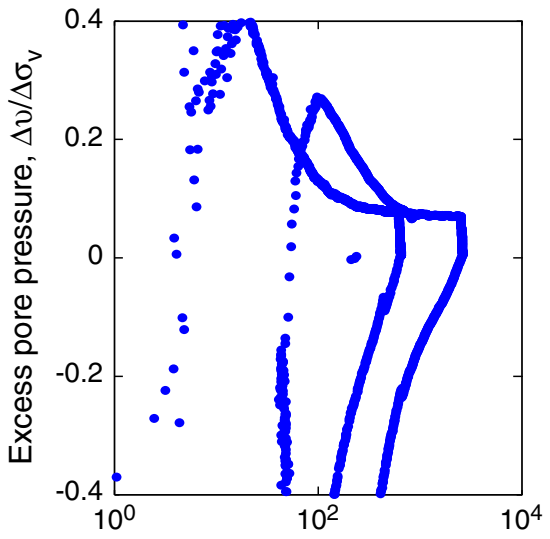

Vertical consolidation stress, $\sigma_{\mathrm{v}}^{\prime}$ (kPa)

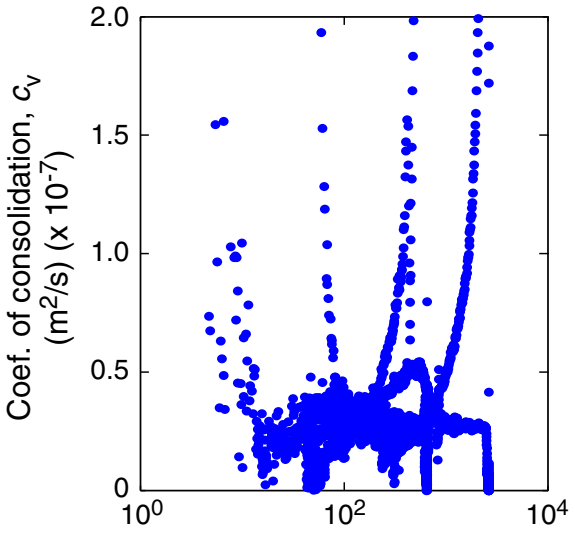

Vertical consolidation stress, $\sigma_{\text {v }}^{\prime}$ (kPa)

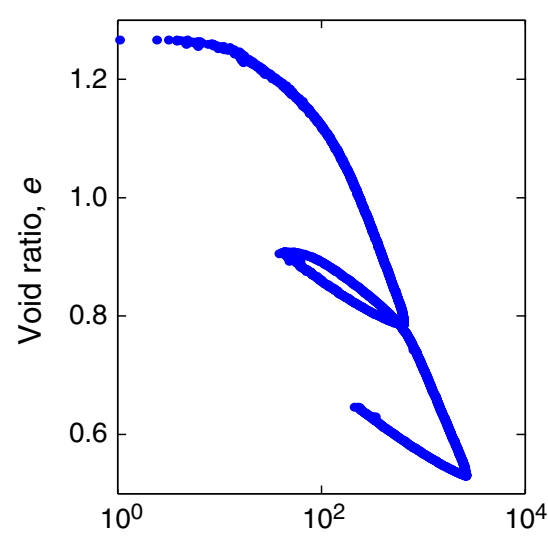

Vertical consolidation stress, $\sigma_{v}^{\prime}$ (kPa)

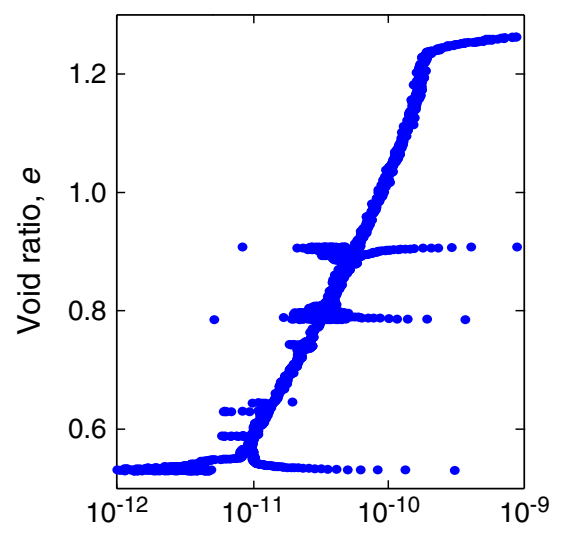

Hydraulic conductivity, $K$ $(\mathrm{m} / \mathrm{s})$ 
Figure F31. CRS015 consolidation data for Sample 308-U1324B-7H-7WR, 60.62 mbsf. Coef. = coefficient.

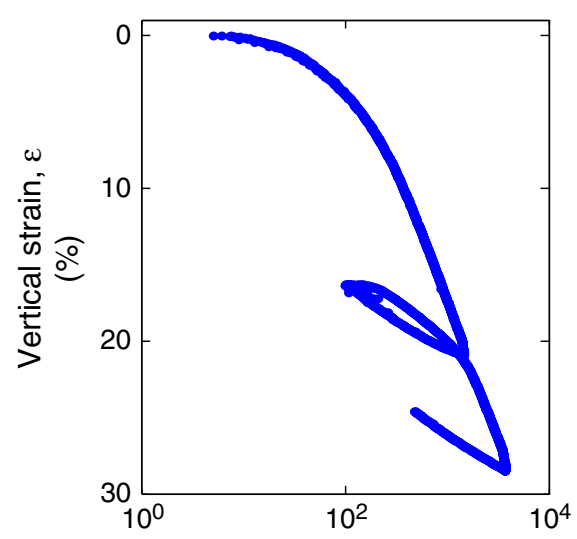

Vertical consolidation stress, $\sigma^{\prime}$ v $(\mathrm{kPa})$

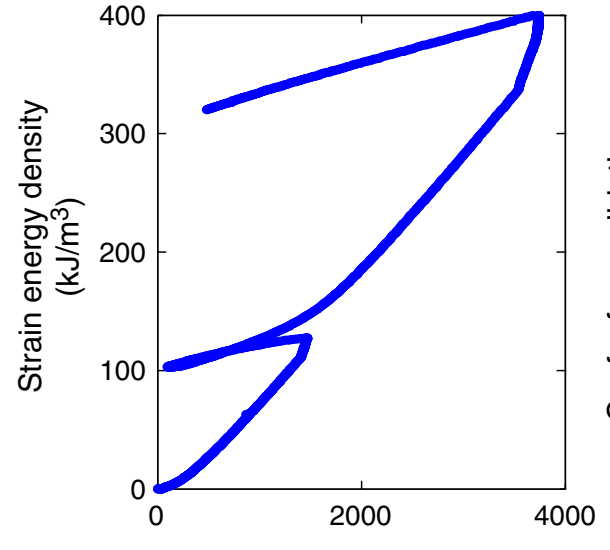

Vertical consolidation stress, $\sigma_{\mathrm{v}}^{\prime}$ (kPa)

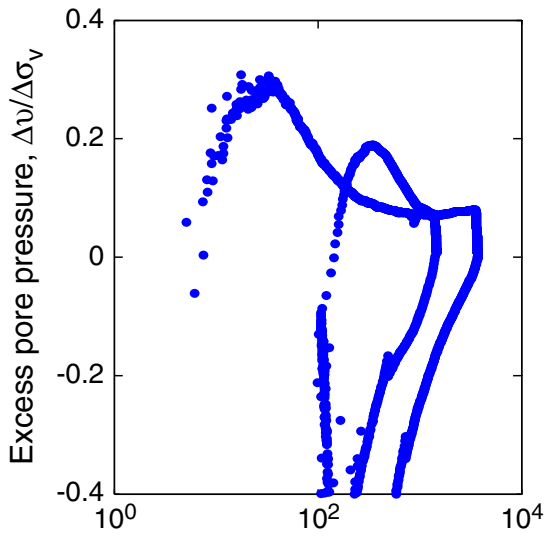

Vertical consolidation stress, $\sigma_{\mathrm{v}}^{\prime}$ (kPa)

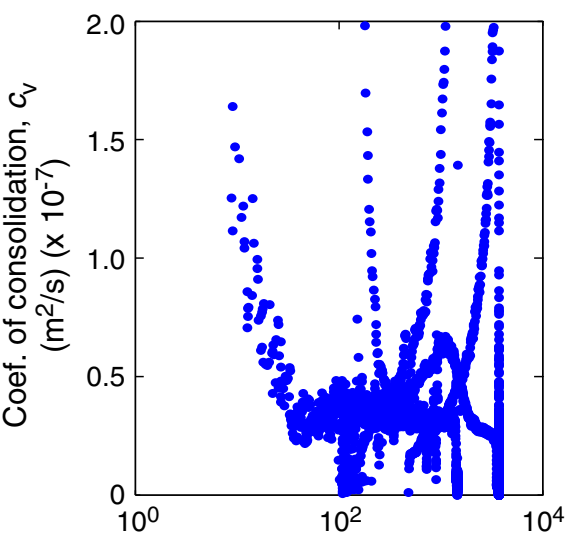

Vertical consolidation stress, $\sigma_{\mathrm{v}}^{\prime}$ (kPa)
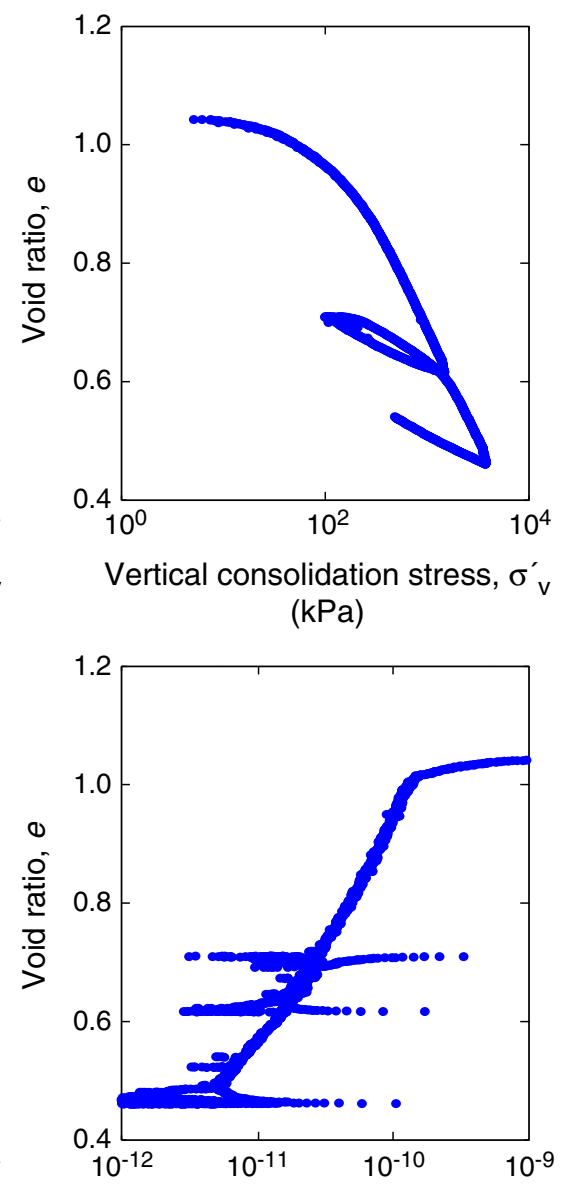

Hydraulic conductivity, $K$ $(\mathrm{m} / \mathrm{s})$ 
Figure F32. CRS018 consolidation data for Sample 308-U1324B-26H-3WR, 220.34 mbsf. Coef. = coefficient.

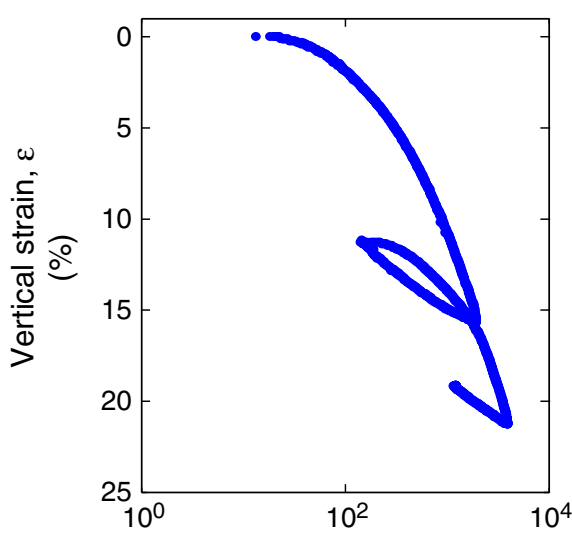

Vertical consolidation stress, $\sigma^{\prime}$, $(\mathrm{kPa})$

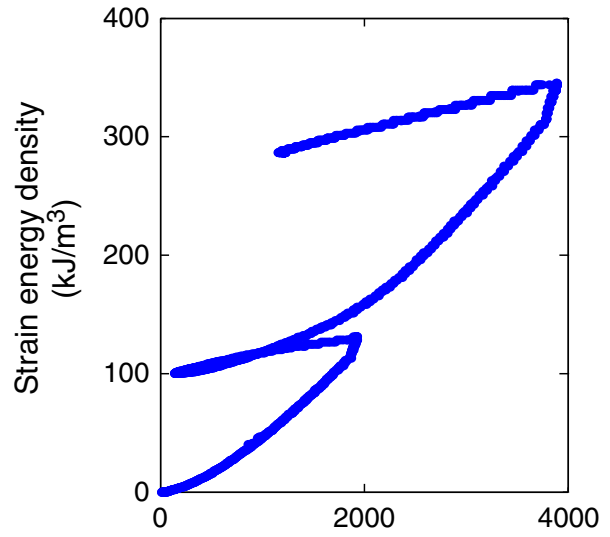

Vertical consolidation stress, $\sigma^{\prime}$ $(\mathrm{kPa})$

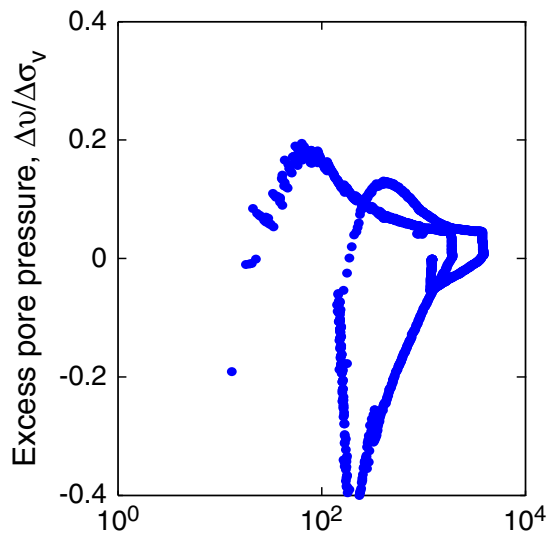

Vertical consolidation stress, $\sigma^{\prime}$ (kPa)

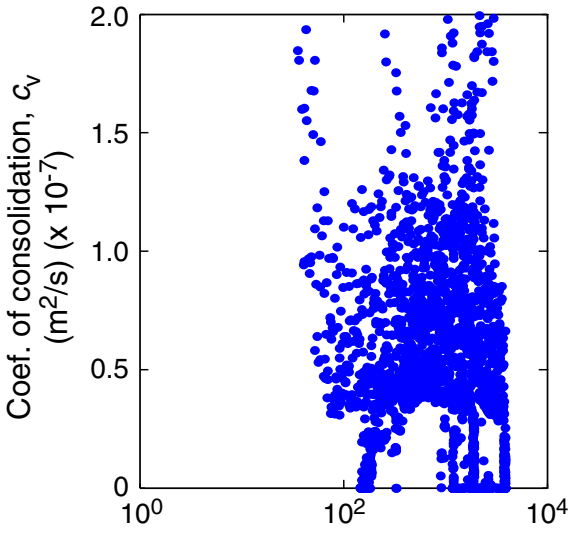

Vertical consolidation stress, $\sigma^{\prime}$ v (kPa)

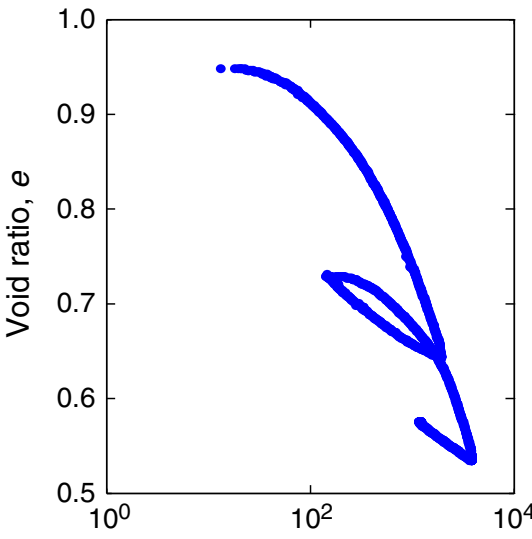

Vertical consolidation stress, $\sigma^{\prime}$ (kPa)

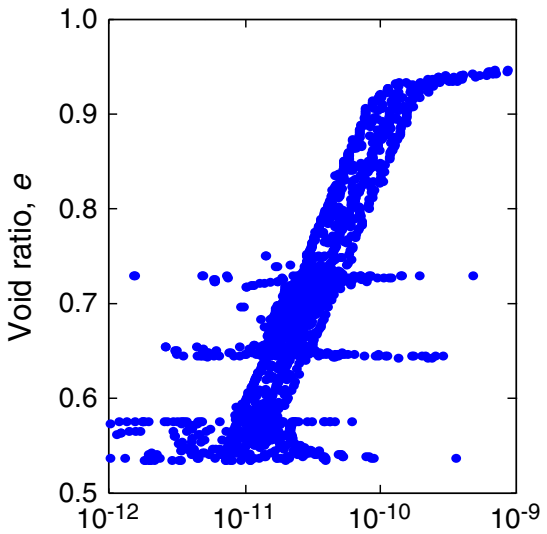

Hydraulic conductivity, $K$ $(\mathrm{m} / \mathrm{s})$ 
Figure F33. CRS019 consolidation data for Sample 308-U1324B-31H-3WR, 261.02 mbsf. Coef. = coefficient.

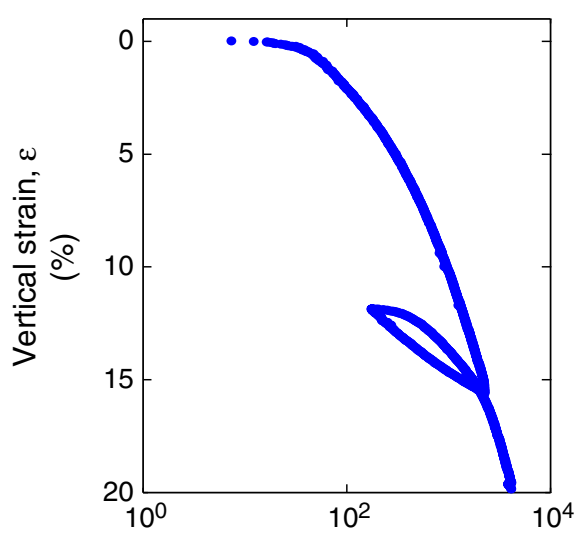

Vertical consolidation stress, $\sigma^{\prime}$ $(\mathrm{kPa})$

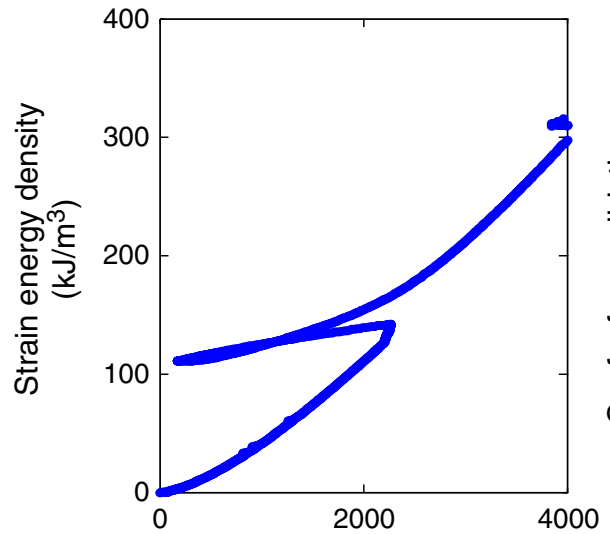

Vertical consolidation stress, $\sigma_{v}^{\prime}$ $(\mathrm{kPa})$

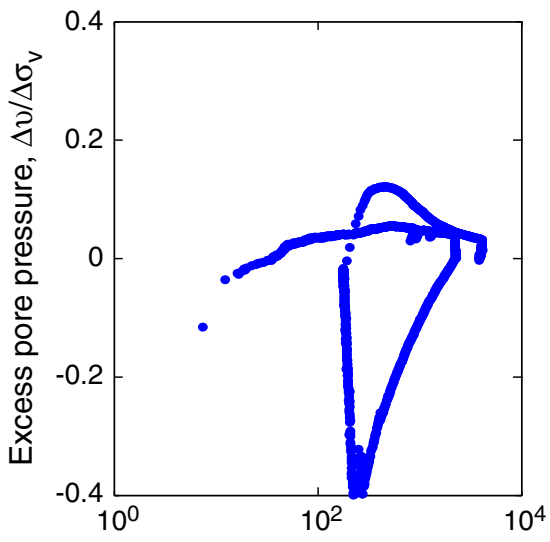

Vertical consolidation stress, $\sigma^{\prime}$ (kPa)

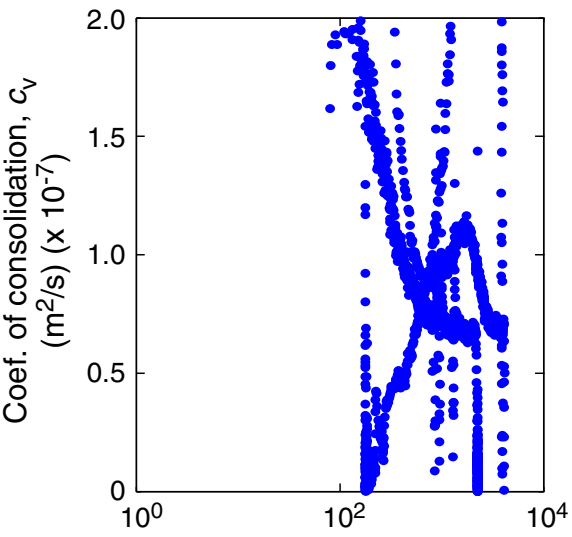

Vertical consolidation stress, $\sigma^{\prime}$ v $(\mathrm{kPa})$

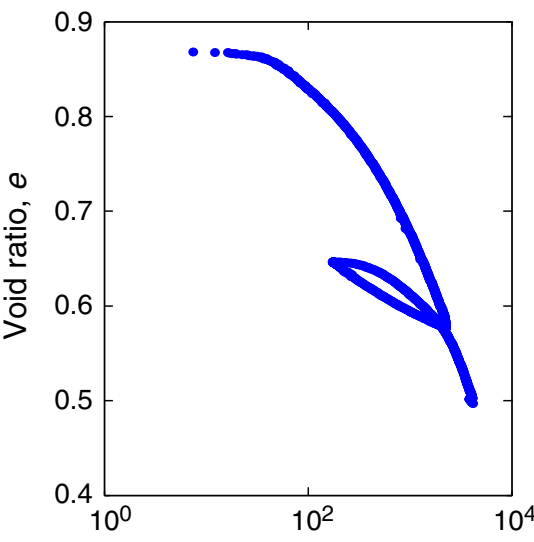

Vertical consolidation stress, $\sigma_{v}^{\prime}$ $(\mathrm{kPa})$

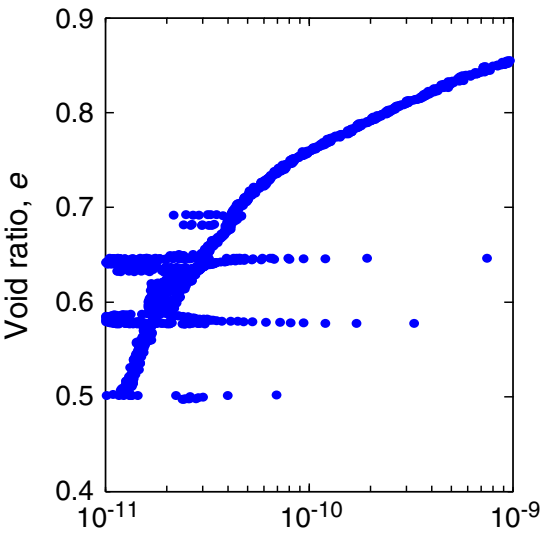

Hydraulic conductivity, $K$ $(\mathrm{m} / \mathrm{s})$ 
Figure F34. CRS020 consolidation data for Sample 308-U1324B-21H-3WR, 183.14 mbsf. Coef. = coefficient.

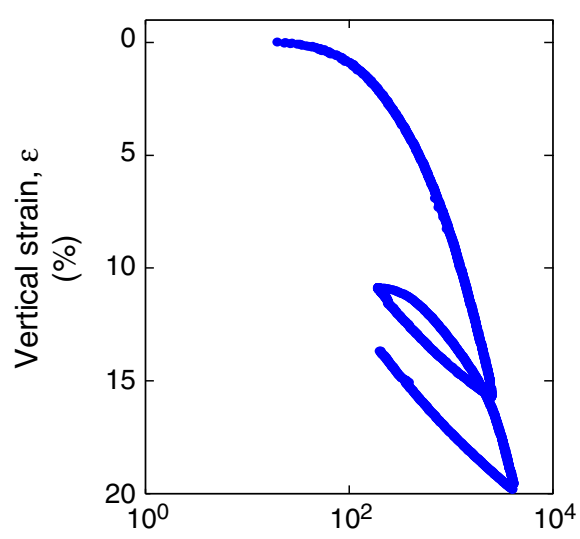

Vertical consolidation stress, $\sigma_{v}^{\prime}$ (kPa)

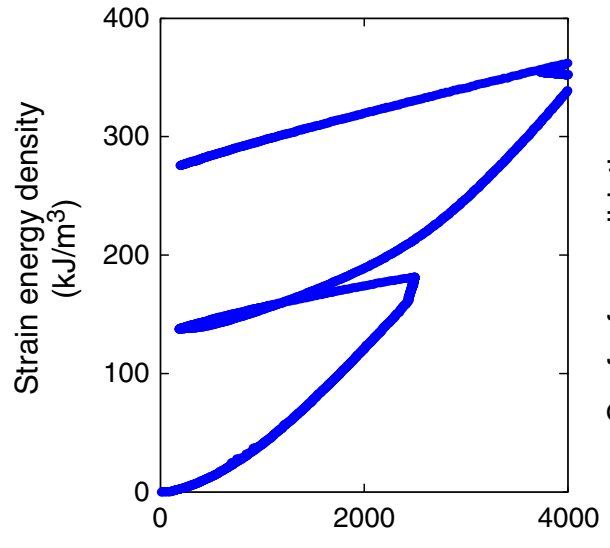

Vertical consolidation stress, $\sigma_{\mathrm{v}}^{\prime}$ (kPa)

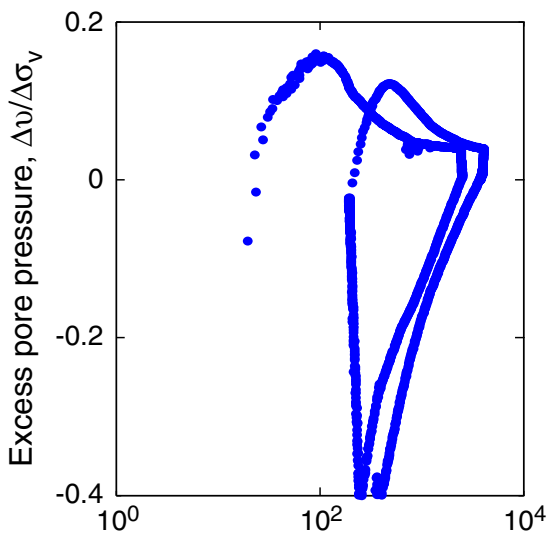

Vertical consolidation stress, $\sigma^{\prime}$, (kPa)

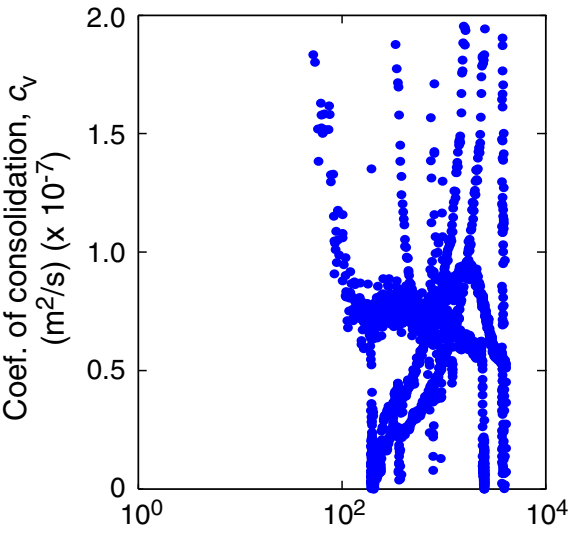

Vertical consolidation stress, $\sigma^{\prime}$ v (kPa)

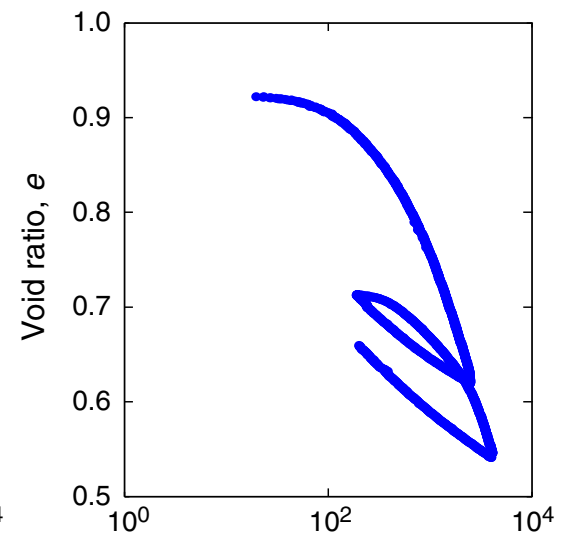

Vertical consolidation stress, $\sigma_{v}^{\prime}$ (kPa)

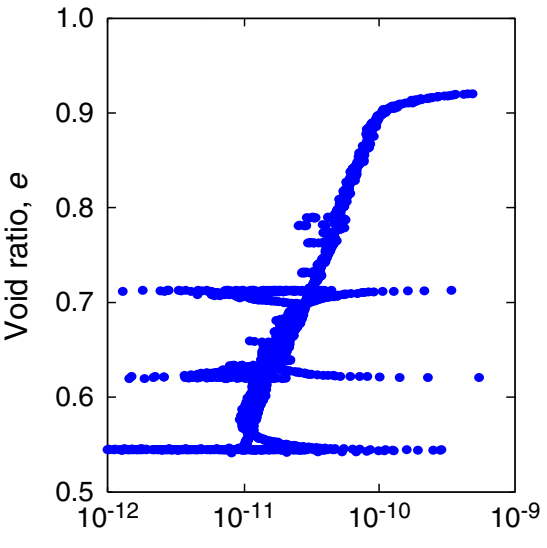

Hydraulic conductivity, $K$ $(\mathrm{m} / \mathrm{s})$ 
Figure F35. CRS021 consolidation data for Sample 308-U1322D-3H-3WR, 103.44 mbsf. Coef. = coefficient.

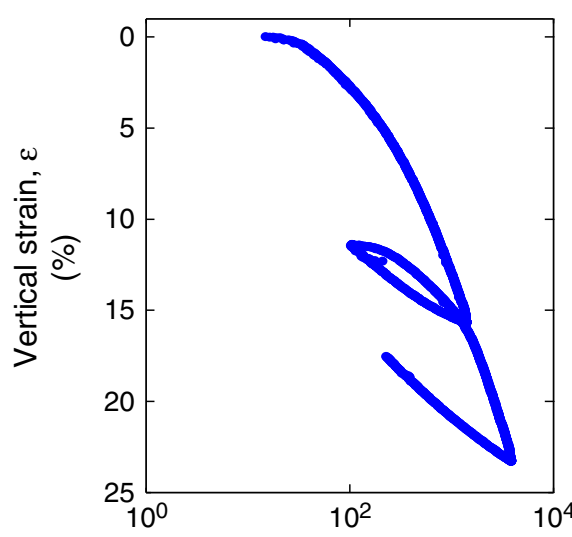

Vertical consolidation stress, $\sigma^{\prime}$, $(\mathrm{kPa})$

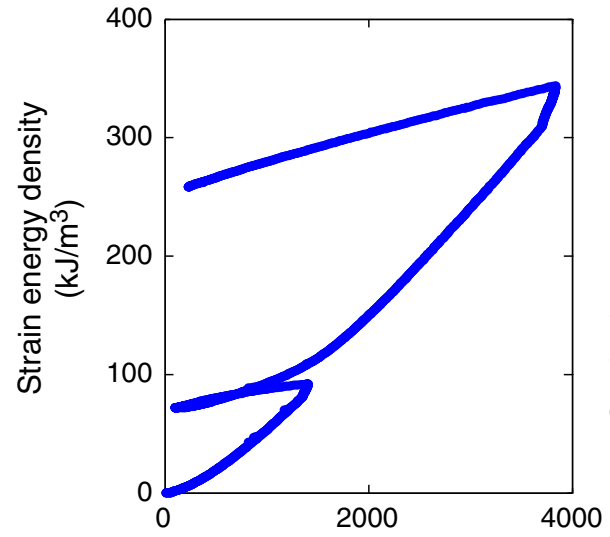

Vertical consolidation stress, $\sigma^{\prime}$ $(\mathrm{kPa})$

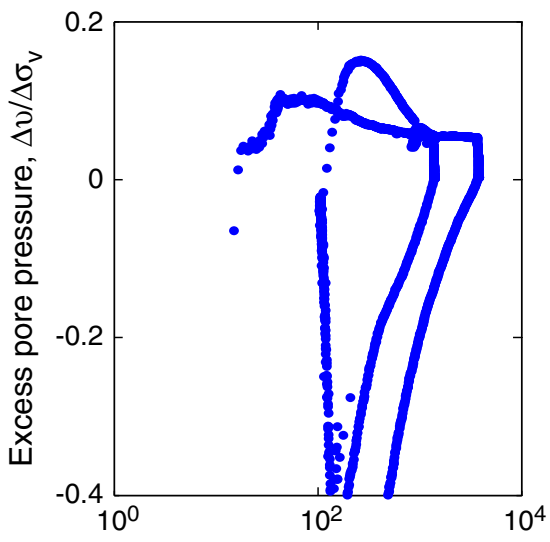

Vertical consolidation stress, $\sigma^{\prime}$ $(\mathrm{kPa})$

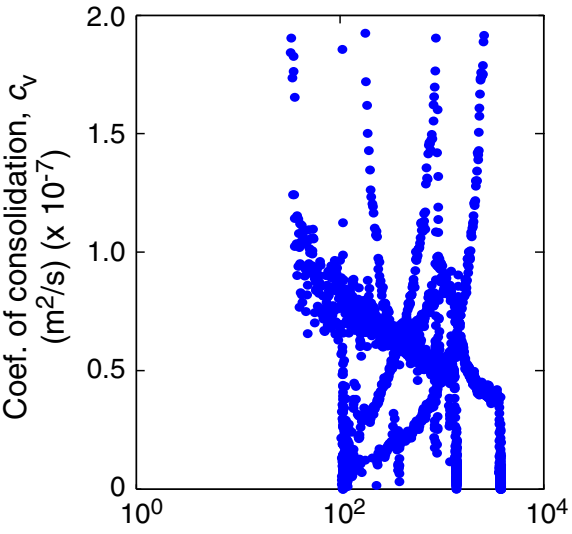

Vertical consolidation stress, $\sigma^{\prime}$ (kPa)

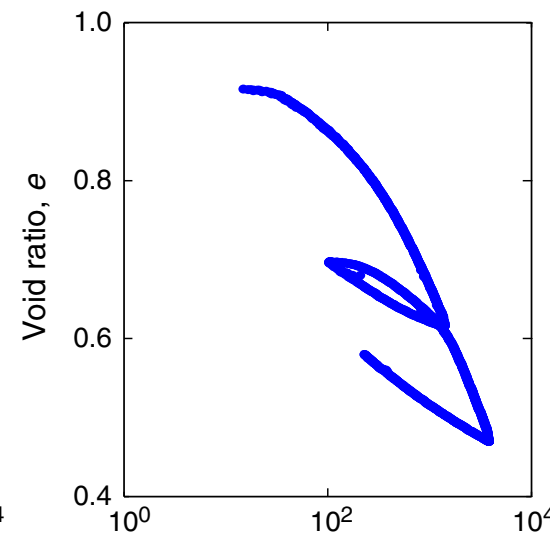

Vertical consolidation stress, $\sigma_{\mathrm{v}}^{\prime}$ (kPa)

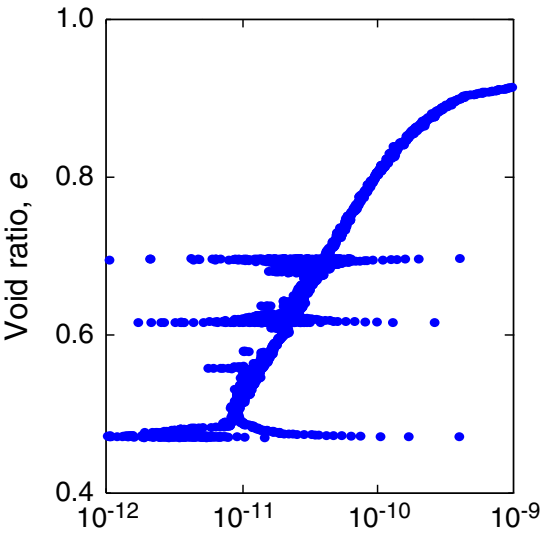

Hydraulic conductivity, $K$ $(\mathrm{m} / \mathrm{s})$ 
Figure F36. Compression and expansion indexes for Ursa Basin sediments (see Table T3).

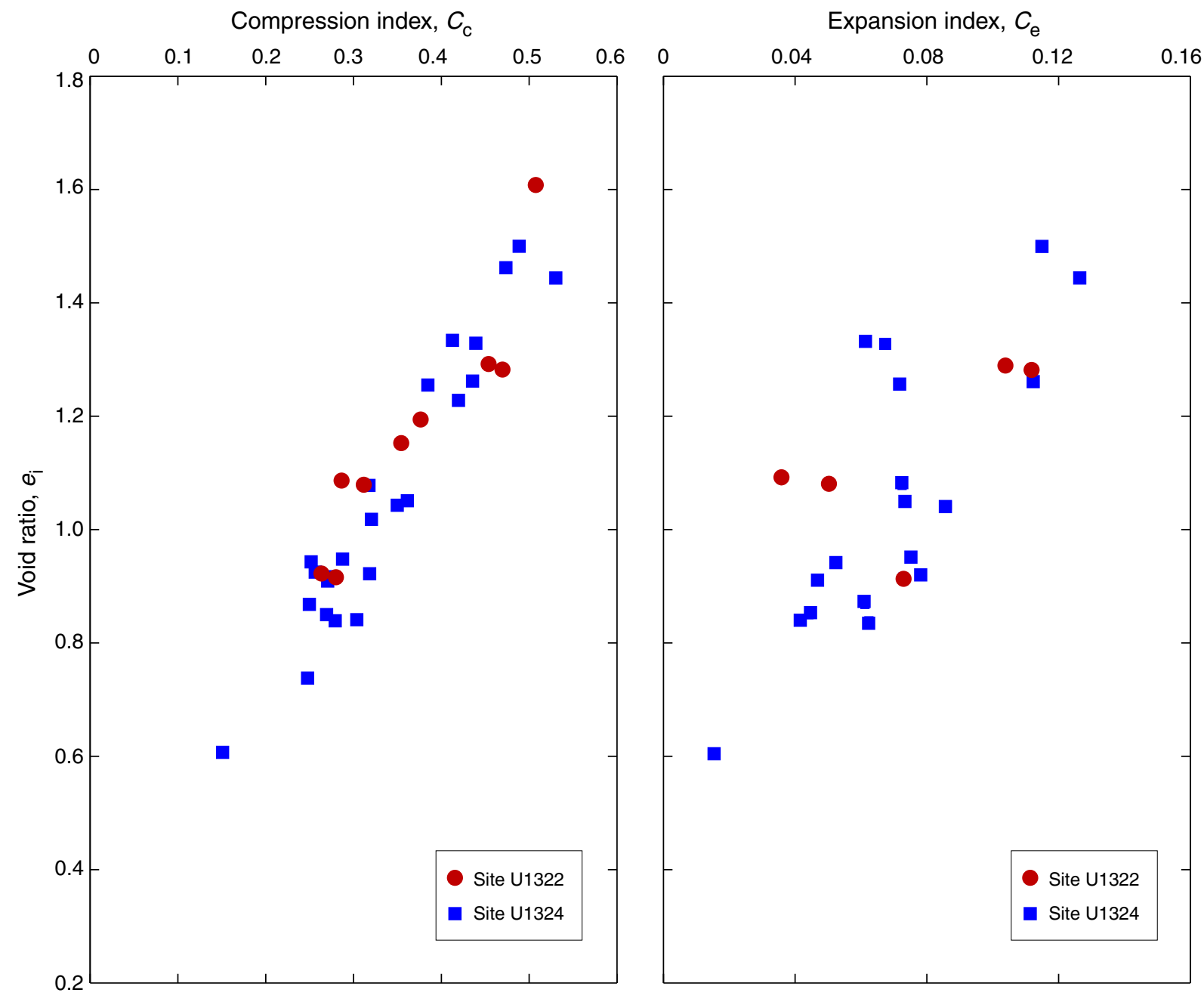


Figure F37. In situ hydraulic conductivity for Ursa Basin sediments (see Table T3).

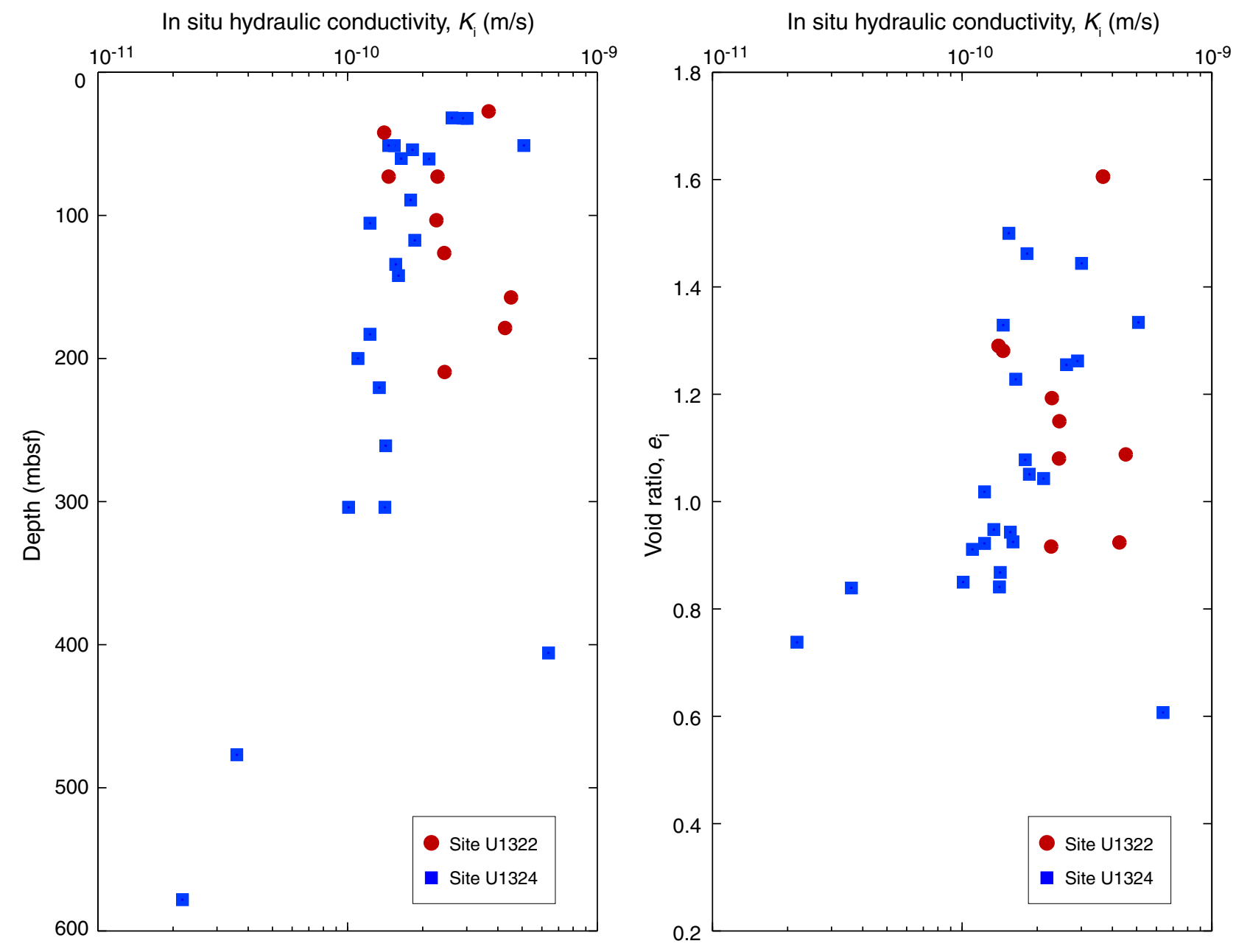


Figure F38. Coefficient of consolidation for Ursa Basin sediments (see Table T3).

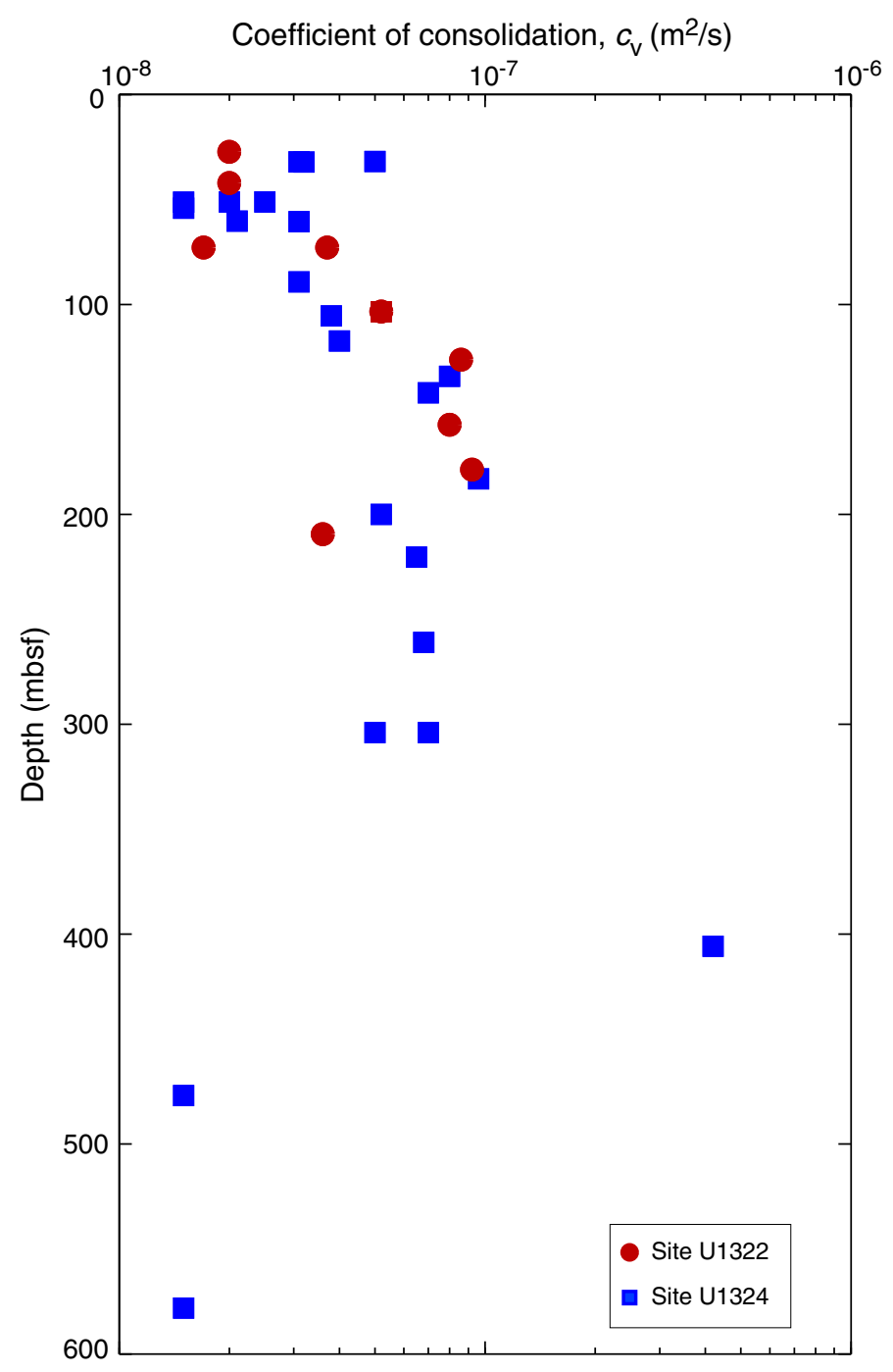


Figure F39. Preconsolidation pressure for Ursa sediments (see Table T3). Preconsolidation stress determined using work-stress method proposed by Becker et al. (1987). Hydrostatic vertical effective stress $\left(\sigma_{\mathrm{vh}}^{\prime}\right)$ calculated using shipboard bulk density data assuming seawater density of $1.024 \mathrm{~g} / \mathrm{cm}^{3}$.

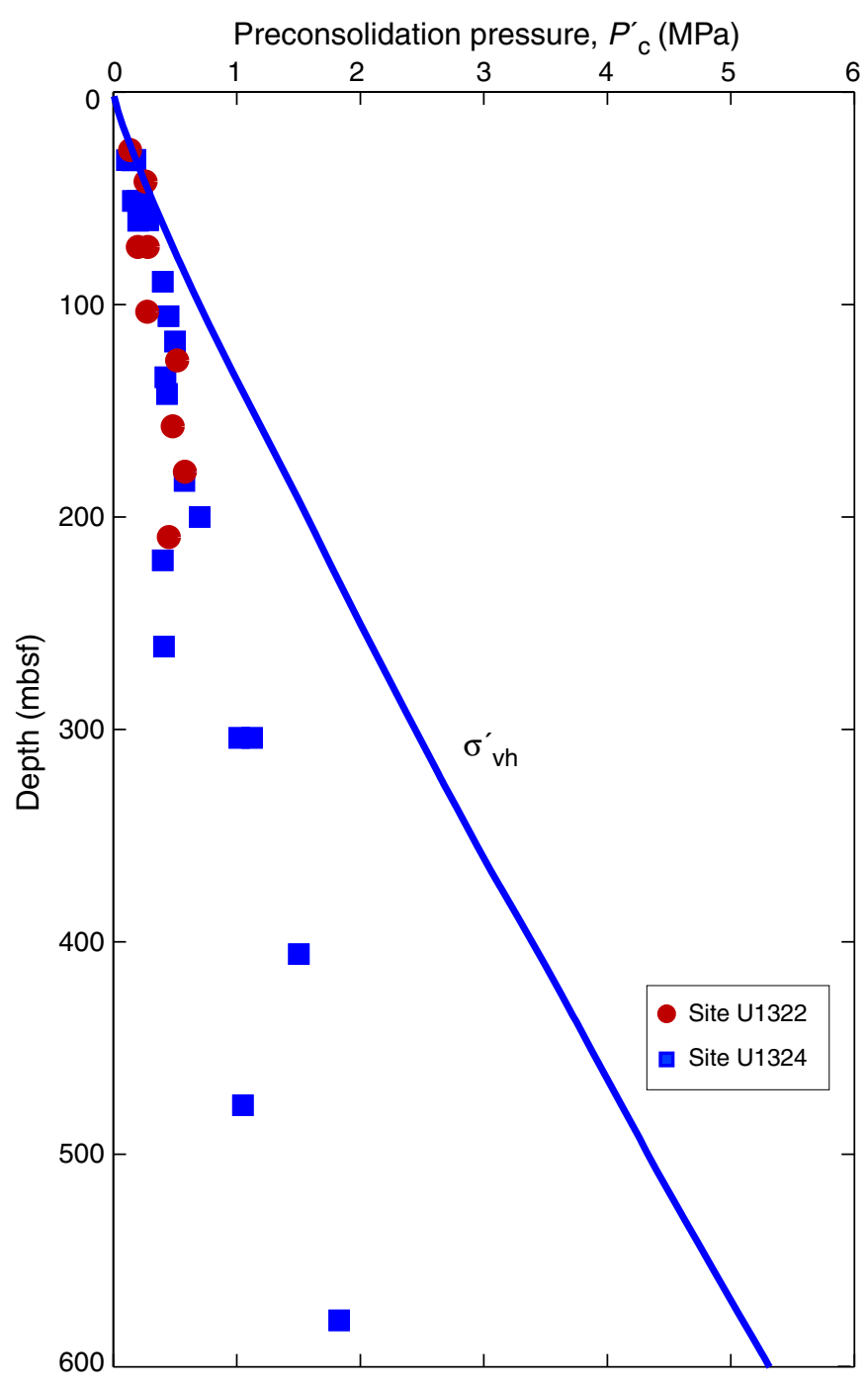


Table T1. Summary of tests conducted on Ursa Basin sediments. (See table notes.)

\begin{tabular}{|c|c|c|c|c|c|c|c|c|}
\hline \multirow{2}{*}{$\begin{array}{l}\text { Core, section, } \\
\text { interval }(\mathrm{cm})\end{array}$} & \multirow{2}{*}{$\begin{array}{l}\text { Depth } \\
\text { (mbsf) }\end{array}$} & \multirow{2}{*}{$\begin{array}{l}\text { Cutting } \\
\text { shoe }\end{array}$} & \multicolumn{2}{|c|}{ Index tests } & \multirow[b]{2}{*}{ X-ray } & \multicolumn{3}{|c|}{ CRSC } \\
\hline & & & WC & PSA & & MIT & PSU & Rice \\
\hline \multicolumn{9}{|l|}{$308-$} \\
\hline U1322B-4H-3WR, 117-137 & 27.17 & Fugro & 1 & 1 & 1 & 1 & & \\
\hline U1322B-15H-1WR, 100-150 & 125.8 & IODP-APC & 1 & 1 & 1 & 1 & & \\
\hline U1322B-18H-6WR, 0-40 & 157.3 & Fugro & 1 & 1 & 1 & 1 & & \\
\hline U1322B-21H-3WR, 112-132 & 178.62 & IODP-APC & 1 & 1 & 1 & 1 & & \\
\hline U1322B-25H-6WR, 97-147 & 209.5 & IODP-APC & 1 & 1 & 1 & 1 & & \\
\hline U1322D-1H-2WR, 50-150 & 42 & Fugro & 1 & 1 & 1 & 1 & & \\
\hline U1322D-2H-2WR, 50-150 & 72 & Fugro & 2 & 2 & 1 & 2 & & \\
\hline U1322D-3H-3WR, 40-140 & 103.4 & Fugro & 1 & 1 & 1 & & & 1 \\
\hline U1324B-4H-7WR, 56-116 & 31.86 & Fugro & 3 & 2 & 2 & 1 & & 2 \\
\hline U1324B-7H-7WR, 0-63 & 60.31 & Fugro & 2 & 2 & 2 & 1 & & 1 \\
\hline U1324B-10H-7WR, 0-45 & 88.8 & IODP-APC & 1 & 1 & 1 & 1 & & \\
\hline U1324B-13H-7WR, 15-35 & 117.24 & Fugro & 1 & 1 & & & 1 & \\
\hline U1324B-15H-5WR, 90-150 & 134.2 & IODP-APCT & 1 & 1 & 1 & 1 & & \\
\hline U1324B-16H-5WR, 90-150 & 142.13 & IODP-APC & 1 & 1 & 1 & 1 & & \\
\hline U1324B-21H-3WR, 112-132 & 183.12 & Fugro & 1 & 1 & 1 & & & 1 \\
\hline U1324B-23H-5WR, 0-22 & 199.8 & Fugro & 1 & 1 & 1 & 1 & & \\
\hline U1324B-26H-3WR, 122-142 & 220.32 & IODP-APC & 1 & 1 & 1 & & & 1 \\
\hline U1324B-31H-3WR, 120-140 & 261 & Fugro & 1 & 1 & 1 & & & 1 \\
\hline U1324B-60X-2WR, 130-150 & 476.7 & IODP-XCB & 1 & 1 & & & 1 & \\
\hline U1324B-70X-6WR, 40-90 & 577.67 & IODP-XCB & 1 & 1 & & & 1 & \\
\hline U1324C-1H-1WR, 110-140 & 51.1 & IODP-APC & 4 & 4 & 1 & 2 & 2 & \\
\hline U1324C-2H-4WR, 0-100 & 104.5 & Fugro & 1 & 1 & 1 & 1 & & \\
\hline U1324C-6H-3WR, 0-106 & 303 & Fugro & 2 & 2 & & & 2 & \\
\hline U1324C-7H-1WR, 50-150 & 405.5 & IODP-APC & 1 & 1 & & & 1 & \\
\hline
\end{tabular}

Notes: See ODP Technical Note 31 (Graber et al., 2002) for coring system information and characteristics. WC = water content measurements, PSA = particle size analysis (Sawyer et al., this volume). X-ray images in $\mathrm{H}$. Nelson et al. (unpubl. data). CRSC = constant rate of strain consolidation. MIT = Massachusetts Institute of Technology, PSU = Pennsylvania State University, Rice $=$ Rice University. IODP-APC $=$ Integrated Ocean Drilling Program advanced piston coring system, IODP-XCB = IODP extended core barrel system, IODP-APCT = IODP advanced piston corer temperature tool. 
Table T2. Nomenclature.

\begin{tabular}{|c|c|c|c|}
\hline Variable & Definition & Dimension & SI unit \\
\hline$C_{c}$ & Compression index & Dimensionless & - \\
\hline$C_{e}$ & Expansion index & Dimensionless & - \\
\hline$G_{s}$ & Grain density & $M / L^{3}$ & $\mathrm{~g} / \mathrm{cm}^{3}$ \\
\hline$H$ & Height of specimen & $\mathrm{L}$ & $\mathrm{mm}$ \\
\hline$H_{0}$ & Initial height of specimen & $\mathrm{L}$ & $\mathrm{mm}$ \\
\hline$K$ & Hydraulic conductivity & $\mathrm{L} / \mathrm{T}$ & $\mathrm{m} / \mathrm{s}$ \\
\hline$K_{\mathrm{i}}$ & In situ hydraulic conductivity & $\mathrm{L} / \mathrm{T}$ & $\mathrm{m} / \mathrm{s}$ \\
\hline OCR & Over consolidation ratio & Dimensionless & - \\
\hline$P_{c}^{\prime}$ & Preconsolidation pressure & $\mathrm{M} / \mathrm{LT}^{2}$ & $\mathrm{kPa}$ \\
\hline SED & Strain energy density & $\mathrm{M} / \mathrm{LT}^{2}$ & $\mathrm{~kJ} / \mathrm{m}^{3}$ \\
\hline$S_{i}$ & Initial saturation & Dimensionless & - \\
\hline$c_{v}$ & Coefficient of consolidation & $\mathrm{L}^{2} / \mathrm{T}$ & $\mathrm{m}^{2} / \mathrm{s}$ \\
\hline$e$ & Void ratio & Dimensionless & - \\
\hline$e_{\mathrm{i}}$ & Initial void ratio measured on specimen & Dimensionless & - \\
\hline$k_{\mathrm{i}}$ & In situ permeability & $\mathrm{L}^{2}$ & $m^{2}$ \\
\hline$m_{v}$ & Frame compressibility & $\mathrm{LT}^{2} / \mathrm{M}$ & $1 / \mathrm{kPa}$ \\
\hline$u$ & Basal pore pressure & $\mathrm{M} / \mathrm{LT}^{2}$ & $\mathrm{kPa}$ \\
\hline$u_{\mathrm{b}}$ & Back pressure & $\mathrm{M} / \mathrm{LT}^{2}$ & $\mathrm{kPa}$ \\
\hline$w_{\mathrm{c}}$ & Water content measured on trimmings & Dimensionless & - \\
\hline$w_{\mathrm{n}}$ & Water content measured on specimen & Dimensionless & - \\
\hline$\Delta u$ & Excess pore pressure & $\mathrm{M} / \mathrm{LT}^{2}$ & $\mathrm{kPa}$ \\
\hline$\Delta u / \sigma_{\mathrm{v}}$ & Normalized excess pore pressure & Dimensionless & - \\
\hline$\delta \varepsilon / \delta \mathrm{t}$ & Strain rate & $1 / \mathrm{T}$ & $\% / h$ \\
\hline$\varepsilon$ & Axial strain & Dimensionless & $\%$ \\
\hline$\varepsilon_{\mathrm{i}}$ & Axial strain prior to compression & Dimensionless & $\%$ \\
\hline$\rho_{\mathrm{b}}$ & Bulk density & $M / L^{3}$ & $\mathrm{~g} / \mathrm{cm}^{3}$ \\
\hline$\gamma_{w}$ & Unit weight of water & $M / L^{2} T^{2}$ & $\mathrm{kN} / \mathrm{m}^{3}$ \\
\hline$\sigma_{\mathrm{v}}$ & Applied vertical stress & $\mathrm{M} / \mathrm{LT}^{2}$ & $\mathrm{kPa}$ \\
\hline$\sigma_{v}^{\prime}$ & Vertical effective stress & $\mathrm{M} / \mathrm{LT}^{2}$ & $\mathrm{kPa}$ \\
\hline$\sigma_{\mathrm{iv}}^{\prime}$ & Vertical effective stress prior to compression & $\mathrm{M} / \mathrm{LT}^{2}$ & $\mathrm{kPa}$ \\
\hline$\sigma_{\text {vh }}^{\prime}$ & Hydrostatic vertical effective stress & $\mathrm{M} / \mathrm{LT}^{2}$ & $\mathrm{kPa}$ \\
\hline$\sigma_{\mathrm{vm}}^{\prime}$ & Maximum vertical effective stress during consolidation & $\mathrm{M} / \mathrm{LT}^{2}$ & $\mathrm{kPa}$ \\
\hline
\end{tabular}




\begin{tabular}{|c|c|c|c|c|c|c|c|c|c|c|c|c|c|c|c|c|c|c|c|c|c|c|c|}
\hline \multirow[b]{2}{*}{$\begin{array}{c}\text { Test } \\
\text { number }\end{array}$} & \multirow[b]{2}{*}{$\begin{array}{l}\text { Hole, core, } \\
\text { section }\end{array}$} & \multicolumn{2}{|c|}{ Depth (mbsf) } & \multirow{2}{*}{$\begin{array}{l}\text { Sample } \\
\text { length } \\
\text { (m) }\end{array}$} & \multicolumn{3}{|c|}{ Index test } & \multicolumn{5}{|c|}{ Specimen data } & \multicolumn{5}{|c|}{ Test conditions } & \multicolumn{6}{|c|}{ Consolidation properties } \\
\hline & & $\begin{array}{l}\text { Core } \\
\text { top }\end{array}$ & Specimen & & $\begin{array}{l}w_{c} \\
(\%)\end{array}$ & SD & N & $\begin{array}{l}w_{\mathrm{n}} \\
(\%)\end{array}$ & $\begin{array}{c}\rho_{\mathrm{b}} \\
(\mathrm{kPa})\end{array}$ & $e_{\mathrm{i}}$ & $\begin{array}{c}S_{i} \\
(\%)\end{array}$ & $\begin{array}{c}G_{\mathrm{s}} \\
\left(\mathrm{g} / \mathrm{cm}^{3}\right)\end{array}$ & $\begin{array}{c}u_{\mathrm{b}} \\
(\mathrm{kPa})\end{array}$ & $\begin{array}{c}\sigma_{\mathrm{iv}}^{\prime} \\
(\mathrm{kPa})\end{array}$ & $\begin{array}{c}\varepsilon_{\mathrm{i}} \\
(\%)\end{array}$ & $\begin{array}{l}\delta \varepsilon / \delta \mathrm{t} \\
(\% / \mathrm{h})\end{array}$ & $\begin{array}{c}u / \sigma_{\mathrm{v}} @ \sigma_{\mathrm{vm}}^{\prime} \\
(\%)\end{array}$ & $C_{c}$ & $C_{e}$ & $\begin{array}{c}P_{c}^{\prime} \\
(\mathrm{kPa})\end{array}$ & $\begin{array}{c}c_{v} \\
\left(\mathrm{~m}^{2} / \mathrm{s}\right)\end{array}$ & $\begin{array}{c}K_{\mathrm{i}} \\
(\mathrm{m} / \mathrm{s})\end{array}$ & $\begin{array}{c}k_{i} \\
\left(\mathrm{~m}^{2}\right)\end{array}$ \\
\hline CRS796 & $1322 \mathrm{D}-2 \mathrm{H}-2$ & 72 & 72.78 & 0.78 & 46.3 & 0.8 & 2 & 47.25 & 1.795 & 1.281 & 102.6 & 2.7 & 384 & 2 & 0.05 & 0.25 & 13.5 & 0.4675 & 0.1114 & 280 & $1.7 \mathrm{E}-8$ & $1.46 \mathrm{E}-10$ & $1.46 \mathrm{E}-17$ \\
\hline CRS797 & $324 \mathrm{C}-1 \mathrm{H}-1$ & 51.1 & & & 42.0 & 12.6 & 2 & 55.69 & 1.731 & 1.500 & 103.2 & 2.7 & 385 & 4 & -0.06 & 0.27 & 12.0 & 0.4887 & 0.1144 & 160 & $1.5 \mathrm{E}-8$ & $1.54 \mathrm{E}-10$ & $1.54 \mathrm{E}-17$ \\
\hline CRS798 & $322 \mathrm{D}-2 \mathrm{H}-2$ & 72 & 2.83 & 83 & 44.9 & 2.9 & 2 & 4.30 & 1.829 & 1.193 & 103.2 & 2.7 & 383 & 3 & 0.06 & 0.30 & 6.0 & 0.3762 & - & 200 & $3.7 \mathrm{E}-8$ & & \\
\hline CRS799 & $1324 \mathrm{C}-1 \mathrm{H}-1$ & 51.1 & 51.31 & 0.21 & 51.2 & 1.3 & 2 & 4.18 & 1.741 & 1.462 & 103.0 & 2.78 & 374 & 6 & 0.03 & 0.27 & 13.0 & .4736 & 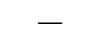 & 224 & $1.5 \mathrm{E}-8$ & $1.82 \mathrm{E}-10$ & $1.82 \mathrm{E}-17$ \\
\hline CRS800 & 1324B-4H-7 & 31.86 & - & - & 47.6 & 6.7 & 2 & 46.20 & 1.803 & & 102.4 & 2.7 & 357 & 3 & 0.06 & 0.30 & 4.8 & 0.3846 & 0.0717 & 180 & $5.0 \mathrm{E}-8$ & $2.62 \mathrm{E}-10$ & $2.62 \mathrm{E}-17$ \\
\hline CRS801 & $1324 \mathrm{~B}-16 \mathrm{H}-5$ & 142.13 & - & - & 33.3 & 0.5 & 2 & 3.77 & 1.932 & 0.925 & 101.5 & & 379 & 3 & 0.00 & 0.2 & & 2564 & - & 435 & $7.0 \mathrm{E}-8$ & 1.60 & $1.59 \mathrm{E}-17$ \\
\hline CRS802 & 1324B-7H-7 & 60.31 & - & - & 44.6 & 1.8 & 2 & 4.42 & 1.802 & 1.228 & 100.6 & 2.7 & 349 & 3 & 0.04 & 0.31 & 11.0 & & - & 283 & $2.1 \mathrm{E}-8$ & & \\
\hline CRS803 & $1324 \mathrm{~B}-15 \mathrm{H}-5$ & 134.2 & - & - & 31.8 & 0.0 & 2 & 34.99 & 1.932 & 0.943 & 103.2 & 2.78 & 378 & 10 & -0.07 & 0.26 & & 0.2517 & 0.0524 & 422 & $8.0 \mathrm{E}-8$ & & $1.56 \mathrm{E}-17$ \\
\hline CRS807 & $-2 \mathrm{H}-4$ & 4.5 & 105.48 & 0.98 & 7.3 & 0.1 & 2 & .89 & 1.872 & 1.018 & 98.0 & & 410 & 16 & -1.25 & 0. & & 204 & - & 448 & $3.8 \mathrm{E}-8$ & & -17 \\
\hline CRS808 & $1322 \mathrm{~B}-15 \mathrm{H}-1$ & 125.8 & 126.28 & & 34.1 & 1.2 & 2 & 7.39 & 1.837 & 1.080 & 96.3 & & 396 & 5 & -0.07 & 0.2 & & & 0.0500 & 516 & $6 E-8$ & & \\
\hline CRS810 & $1322 \mathrm{~B}-18 \mathrm{H}-6$ & 157.3 & 157.42 & & 32.5 & 0.1 & 2 & 34.48 & 1.790 & 1.088 & 88.1 & 2.7 & 423 & 12 & -0.15 & 0.21 & 2. & & 0.0359 & 480 & & & \\
\hline CRS812 & $-23 \mathrm{H}-5$ & 199.8 & 200 & & 0.9 & 0.6 & 2 & 01 & 920 & 0.5 & 97.7 & & 42 & 13 & -0.23 & 0. & & & 0.0470 & 700 & $2 E-8$ & & \\
\hline CRS813 & $1324 \mathrm{~B}-10 \mathrm{H}-7$ & 88.8 & 89.22 & & 37.8 & 3.3 & 2 & 3.12 & 1.848 & 1.078 & 98.3 & 2.7 & 424 & 18 & -0.09 & 0.21 & & .3175 & 0.0725 & 400 & $3.1 \mathrm{E}-8$ & & $=-17$ \\
\hline CRS815 & $1322 \mathrm{~B}-4 \mathrm{H}-3$ & 27.17 & 7.21 & & 55.1 & 2.9 & 2 & 8.42 & 1.690 & 1.606 & 101.1 & 2.78 & 424 & 5 & -0.16 & 0.1 & & & - & 135 & $2.0 \mathrm{E}-8$ & & \\
\hline CRS824 & $3-25 \mathrm{H}-6$ & 209.5 & 209. & & 32.8 & 0.6 & 3 & & 739 & & 83.4 & & & 5 & -1.53 & & & & - & 450 & E-8 & & \\
\hline CRS825 & $1322 \mathrm{~B}-21 \mathrm{H}-3$ & 178.62 & 178.7 & & 28.3 & 0.5 & 3 & 30.39 & 1.884 & 0.924 & 91.5 & 2.7 & 403 & 9 & -0.09 & 0.1 & & 2627 & - & 580 & $9.2 \mathrm{E}-8$ & & $4.27 \mathrm{E}-17$ \\
\hline CRS826 & 1322D-1H-2 & 42 & 42.87 & & 44.6 & 1.8 & 3 & 45.21 & 1.763 & 1.29 & 97.4 & 2.7 & 387 & 5 & 0.05 & 0.21 & & 455 & 0.1038 & 260 & $2.00 \mathrm{E}-8$ & & \\
\hline CRS001 & $-6 \mathrm{H}-3$ & 303 & 304 & & & 0.20 & 4 & - & - & $0.84^{*}$ & & 2.7 & 300 & 112 & -0.03 & 0. & & & 0.0421 & 1124 & $7.0 \mathrm{E}-8$ & & $1.41 \mathrm{E}-17$ \\
\hline CRSO02 & 1324 & 303 & 303. & & & 1.40 & 3 & - & - & $0.85^{*}$ & & & 300 & 112 & -0.14 & 0. & & 2694 & 0.0442 & 1020 & $5.0 \mathrm{E}-8$ & & $E-17$ \\
\hline CRSO03 & $1324 \mathrm{C}-1 \mathrm{H}-1$ & 51.1 & & & 50 & 1.80 & 3 & - & - & $1.33^{*}$ & & 2. & 300 & 7 & -1.59 & 0.15 & 4 & 126 & 0.0365 & 197 & $E-8$ & & \\
\hline CRS004 & $324 \mathrm{C}-1 \mathrm{H}-1$ & & & & & 2.90 & 3 & - & - & $1.33^{*}$ & & & & 8 & -0.41 & 0. & & & 0.06 & 232 & & & \\
\hline CRS005 & $1324 \mathrm{~B}-13 \mathrm{H}-7$ & 117.24 & $4 \quad 117.4$ & & 40 & 1.40 & 3 & 39.700 & 1.867 & 1.05 & 101.60 & 2.7 & & 80 & 0.69 & 0.2 & & 3613 & 0.0734 & 500 & $4.0 \mathrm{E}-8$ & $E-10$ & $1.86 \mathrm{E}-17$ \\
\hline CRS006 & 1324B-70X-6 & 577.67 & 578.13 & 0.46 & 26 & 0.60 & 2 & 28.000 & 2.018 & 0.74 & 102.10 & 2.74 & 320 & 50 & -0.38 & 0.45 & 11.0 & 2478 & - & 1829 & $1.5 \mathrm{E}-8$ & BE-11 & $2.18 \mathrm{E}-18$ \\
\hline & & 476.7 & 476.86 & & 30 & 0.70 & 3 & & 1.946 & & 98.00 & & & 50 & -0.66 & 0.3 & & & & 1050 & & & \\
\hline CRS008 & 1324C-7H-1 & 405.5 & & & 23 & 0.60 & 3 & 19.900 & 2.040 & 0.61 & 87.90 & 2.74 & & 40 & 0.84 & 0. & & .1508 & 0.0153 & 1502 & $4.2 \mathrm{E}-7$ & BE-10 & $6.38 \mathrm{E}-17$ \\
\hline CRS013 & 1324B-4H-7 & 31.86 & 32.14 & 0.84 & 48.1 & 0.3 & 4 & 53.55 & 1.697 & 1.444 & 100.16 & 2.7 & 387 & 6 & -0.03 & 0.30 & 6.59 & 0.5303 & 0.1267 & 154 & $3.2 \mathrm{E}-8$ & $3.01 \mathrm{E}-10$ & $3.01 \mathrm{E}-17$ \\
\hline CRS014 & & 31.86 & 32.10 & & 45.4 & 1.3 & 4 & 47.18 & 1.757 & 1.262 & 100.93 & 2.7 & 386 & 2 & -0.19 & 0. & & & 0.1123 & 112 & $3.1 \mathrm{E}-8$ & & $2.90 \mathrm{E}-17$ \\
\hline CRS015 & 1324B-7H-7 & 60.31 & & & & 6.4 & 4 & & 1.837 & 1.043 & 101.05 & 2.7 & & 1 & 0.00 & & & 198 & 0.0862 & 202 & $3.1 \mathrm{E}-8$ & $2.12 \mathrm{E}$ & $2.12 \mathrm{E}-17$ \\
\hline CRS018 & $1324 \mathrm{~B}-26 \mathrm{H}-3$ & 220.32 & 220.34 & 1.24 & 34.4 & 0.4 & 4 & 34.86 & 1.869 & 0.948 & 99.24 & 2.7 & 386 & 13 & 0.00 & 0.30 & 4.31 & 0.2875 & 0.0749 & 400 & $6.5 \mathrm{E}-8$ & $1.34 \mathrm{E}-10$ & $1.34 \mathrm{E}-17$ \\
\hline CRS019 & $1324 \mathrm{~B}-31 \mathrm{H}-3$ & 261 & 261.02 & & 29.6 & 0.1 & 4 & 26.46 & 1.871 & 0.868 & 91.65 & 2.7 & 387 & 7 & -0.01 & 0. & & 0.2497 & 0.0612 & 410 & $6.8 \mathrm{E}-8$ & & $1.42 \mathrm{E}-17$ \\
\hline CRSO20 & $1324 \mathrm{~B}-21 \mathrm{H}-3$ & 83.12 & & & 32.9 & 1.6 & 4 & & 1.884 & & & 2.7 & 387 & 20 & -0.01 & 0.30 & & & 0.0777 & 576 & $9.6 \mathrm{E}-8$ & $1.23 \mathrm{E}-10$ & $1.23 \mathrm{E}-17$ \\
\hline CRS021 & $1322 \mathrm{D}-3 \mathrm{H}-3$ & 103.4 & 103.44 & 0.44 & 31.7 & 1.0 & 4 & 32.91 & 1.873 & 0.916 & 97.03 & 2.7 & 387 & 15 & 0.00 & 0.30 & 4.52 & 0.2749 & 0.0727 & 274 & $5.2 \mathrm{E}-8$ & $2.27 \mathrm{E}-10$ & $2.27 \mathrm{E}-17$ \\
\hline
\end{tabular}

Notes: All sections taken from whole rounds. See Table T2 for variable definitions. SD = standard deviation, $N=$ number of observations. ${ }^{*}=$ water content not measured and $e_{\mathrm{i}}$ calculated from $w_{\mathrm{c}}$ assuming grain density of $2.74 \mathrm{~g} / \mathrm{cm}^{3}$. For other tests, $e_{\mathrm{i}}$ calculated from $w_{\mathrm{n}}$ assuming grain density of $2.74 \mathrm{~g} / \mathrm{cm}^{3}$ (Pennsylvania State University [PSU]), $2.78 \mathrm{~g} / \mathrm{cm}^{3}$ (Massachusetts Institute of Technology [MIT]), and $2.70 \mathrm{~g} / \mathrm{cm}^{3}$ (Rice University [Rice]). Tests CRS796-CRS826 performed at MIT, CRS01-CRS08 at PSU, and CRS13-CRS21 at Rice. 
Table T4. Consolidation data file headers.

\begin{tabular}{cccccccccccc}
\hline $\begin{array}{c}\text { Time } \\
(\mathrm{s})\end{array}$ & $\begin{array}{c}e \\
(\%)\end{array}$ & $\begin{array}{c}\sigma_{\mathrm{v}} \\
(\mathrm{kPa})\end{array}$ & $\begin{array}{c}u \\
(\mathrm{kPa})\end{array}$ & $\begin{array}{c}u_{\mathrm{b}} \\
(\mathrm{kPa})\end{array}$ & $\begin{array}{c}\sigma_{\mathrm{v}}^{\prime} \\
(\mathrm{kPa})\end{array}$ & $e$ & $\begin{array}{c}\Delta u \\
(\mathrm{kPa})\end{array}$ & $\begin{array}{c}K \\
(\mathrm{~m} / \mathrm{s})\end{array}$ & $\begin{array}{c}c_{\mathrm{v}} \\
\left(\mathrm{m}^{2} / \mathrm{s}\right)\end{array}$ & $\begin{array}{c}\text { SED } \\
\left(\mathrm{kJ} / \mathrm{m}^{3}\right)\end{array}$ \\
\hline 154750 & -0.0302 & 120.169 & 281.752 & 299 & 120.001 & 0.8415 & 0.252 & $0.00 \mathrm{E}+00$ & $0.00 \mathrm{E}+00$ & 0.0021 & 0 \\
155750 & -0.0148 & 140.032 & 283.899 & 300 & 138.4327 & 0.8412 & 2.399 & $3.53 \mathrm{E}-10$ & $1.29 \mathrm{E}-06$ & 0.0171 & 0.0199 \\
156740 & 0.0556 & 151.746 & 283.04 & 301 & 150.7193 & 0.8399 & 1.54 & $1.09 \mathrm{E}-09$ & $1.40 \mathrm{E}-06$ & 0.0101 & 0.1217 \\
157750 & 0.1556 & 162.441 & 285.188 & 301 & 159.9823 & 0.8381 & 3.688 & $4.80 \mathrm{E}-10$ & $5.22 \mathrm{E}-07$ & 0.0227 & 0.2772 \\
158740 & 0.2364 & 171.608 & 283.899 & 300 & 170.0087 & 0.8366 & 2.399 & $7.94 \mathrm{E}-10$ & $7.49 \mathrm{E}-07$ & 0.014 & 0.4107 \\
159740 & 0.3493 & 181.794 & 287.335 & 301 & 177.904 & 0.8345 & 5.835 & $3.42 \mathrm{E}-10$ & $2.49 \mathrm{E}-07$ & 0.0321 & 0.6076 \\
160750 & 0.4416 & 189.434 & 288.624 & 301 & 184.6847 & 0.8328 & 7.124 & $2.66 \mathrm{E}-10$ & $1.71 \mathrm{E}-07$ & 0.0376 & 0.7758 \\
161740 & 0.5433 & 194.017 & 287.335 & 301 & 190.127 & 0.8309 & 5.835 & $3.23 \mathrm{E}-10$ & $1.80 \mathrm{E}-07$ & 0.0301 & 0.9671 \\
\hline
\end{tabular}

Note: See Table T2 for variable definitions. 